1959 Clinicopathological Significance of Early-Stage Lung Adenocarcinoma with Micropapillary Component: Associations with Prognosis, EGFR and KRAS Gene Mutations

A Yoshizawa, S Sumiyoshi. Shinshu University Hospital, Matsumoto, Nagano, Japan; Kyoto University Hospital, Kyoto, Japan.

Background: Lung adenocarcinoma with micropapillary component (LA-MPC) is known to have biologically aggressive behavior and its predominant tumor was listed up as a new entity of invasive adenocarcinoma by the International Association for the Study of Lung Cancer, American Thoracic Society, and European Respiratory Society. The aim of this study was to evaluate the clinicopathological characteristics of earlystage LA-MPC and to investigate correlations between LA-MPC and the EGFR or, KRAS mutation status.

Design: We retrospectively reviewed 440 LA patients who underwent resection. We defined LA-MPC as adenocarcinoma with MPC occupying at least $5 \%$ of the entire tumor. EGFR and KRAS mutations were detected using the established methods.

Results: Of 440 cases, 256 cases were classified as Stage IA cases. Of which, 53 cases (20.7\%) had MPC. The disease-free 5-year survival rates of the MPC-negative and MPC-positive groups in stage IA tumors were $92.1 \%$ and $77.6 \%$, respectively, and there was statistically significant difference between the groups $(p=0.003)$. On the other hand, the overall 5-year survival rates of the MPC-negative and MPC-positive groups in stage IA tumors were $91.8 \%$ and $92.3 \%$, respectively, showing no statistically significant difference $(p=0.973)$. Recurrent rates of LA-MPC were significantly higher $(\mathrm{n}=10,17.8 \%)$ than cases without MPC $(4.4 \%)(\mathrm{p}<0.001)$. No KRAS mutation was detected in all the ten cases. Of the six alive cases with disease, EGFR mutations were detected in 5 cases $(83.3 \%$ ) and the four of them were treated with a tyrosine kinase inhibitor (TKI) with long survival (median: 64.6 months). Additionally, one case with EGFR mutation was detected in four dead cases, who died 100.6 months later after initial resection with using TKI.

Conclusions: LA-MPC, defined as occupying $5 \%$ or more of the entire tumor, was associated with a strongly invasive nature and was a prognostic factor in early-stage patients. Moreover LA-MPCs were biologically aggressive but could be controlled by EGFR-TKIs.

1960 The Histopathology of End Stage Pulmonary Sarcoidosis: Comparison of 11 Explanted Lungs with Usual Interstitial Pneumonia

C Zhang, K Chan, HAmes, J Myers, L Schmidt. University of Michigan, Ann Arbor, MI. Background: Sarcoidosis is an idiopathic multi-system disease that commonly affects the respiratory tract and is characterized by nonnecrotizing epithelioid granulomas. In 10 to $30 \%$ of cases the lungs undergo progressive fibrosis resulting in respiratory failure. Pathologic features of end stage pulmonary sarcoidosis (ESPS) have not been well described; anecdotal reports have suggested that it may mimic usual interstitial pneumonia (UIP). We hypothesized that ESPS has distinct histologic features.

Design: We identified 11 patients with a clinical diagnosis of ESPS who underwent lung transplantation between 1995 and 2012. Controls were 10 age and sex matched lung transplant patients with UIP (IPF=8; systemic lupus erythematosis=2). H\&E-stained sections of each case were examined for the following features: extent/pattern of fibrosis; presence/quantity (per 10 high power fields) of fibroblastic foci and granulomas; distribution and morphology of granulomas; presence of granulomas in hilar lymph nodes; presence/extent of honeycomb change; bronchiectasis; hypertensive vascular change. Extent of fibrosis and honeycomb change were scored as follows: $1=1-25 \%$; $2=26-50 \% ; 3=51-75 \% ; 4=76-100 \%$ of lung parenchyma.

Results: Well-formed granulomas with a lymphangitic distribution (visceral pleura, bronchovascular bundles, and interlobular septa) were seen in all ESPS cases, but none of the control cases. Granulomas were present in hilar lymph nodes from 9 of 9 ESPS cases, and none of 8 control cases. Ten of 11 ESPS cases showed patchy fibrosis with a lymphangitic distribution; 7 of 10 control cases showed patchy fibrosis but in a random distribution. One of the 11 ESPS cases showed diffuse fibrosis, while diffuse fibrosis was seen in 3 of 10 control cases ( $9 \%$ vs $30 \%, p<0.05)$. The average extent of fibrosis was significantly lower in ESPS cases, as compared with that in control cases $(2.5 \pm$ 0.5 vs $3.5 \pm 0.5, \mathrm{p}<0.05$ ). There was no significant difference in honeycomb changes, fibroblastic foci, bronchiectasis and hypertensive vascular changes between the ESPS group and control group.

Conclusions: ESPS and UIP have distinct histopathologic features in explanted lungs. ESPS is characterized by a combination of well-formed granulomas and a lymphangitic pattern of fibrosis that differs from the patchwork pattern of fibrosis seen in UIP. Granulomas in hilar lymph nodes are limited to patients with ESPS.

\section{Quality Assurance}

\section{Evaluation of ER, PR and Her2 in Breast Carcinoma Metastatic to Bone: A Comparison of Results between FNA Cell Blocks and Surgical Biopsies}

G Aggarwal, J Magda, ME Arcila, O Lin, M Edelweiss. Memorial Sloan-Kettering Cancer Center, New York, NY.

Background: Immunohistochemical profile (IHC) is critical in the management of patients with metastatic breast carcinoma. Accurate detection of ER, PR and Her2 require strict adherence to $\mathrm{ASCO} / \mathrm{CAP}$ guidelines. The decalcification process (decal) performed in surgical biopsies (SB) of bone metastasis (bmets) has the potential to cause IHC alterations and delayed reporting. Cell blocks (CB) obtained from FNA of bmets represent a viable alternative. In the present study we aimed to compare ER, PR and Her2 IHC results on formalin-fixed CB to those on concurrent formalin-fixed decalcified SB.
Design: Thirty nine archival FNA samples with concurrent SB from patients with bmets were identified over a period of 12 months. Tumor cells were present in 31/39 CB $(80 \%)$ of which $22 / 31(71 \%)$ had more than 20 tumor cells. Archival blocks or IHC stains were available in $27 \mathrm{CB} / \mathrm{SB}$ pairs. IHC for ER, PR, and Her2 were performed/ evaluated on these $27 \mathrm{CB}$ and compared to those of the paired SB. Interpretation of ER and PR IHC included intensity of nuclear staining (weak, moderate, strong), and \% of stained tumor cells. Her2 IHC scoring, was evaluated for complete membranous staining, uniformity (present or absent) and intensity of staining pattern. This semiquantitative scoring system was used for both $\mathrm{CB}$ and $\mathrm{SB}$.

Results: Discrepant IHC was found in $4 / 27$ cases in which the $\mathrm{CB}$ showed cells positive for ER (ranging from 5-98\% of the cells) while no ER staining was seen in the matching $\mathrm{SB}(\mathrm{p}=0.29)$. Interestingly, crush artifact was noted in 3 of these $4 \mathrm{SB}$ cases. ER was originally positive in the primary breast carcinoma in 3 of such cases, while negative in 1 . Seven (35\%) of the remaining 20 ER positive SB cases showed a lower \% of ER positive tumor cells ( $>30 \%$ difference), as well as, decreased intensity of staining when compared to CB. Overall, comparison of ER staining (including number of discordant cases, decrease in intensity and \% of stained tumor cells in SB) showed significant difference of results between $\mathrm{CB}$ and $\mathrm{SB}(\mathrm{p}=0.0002)$. PR and Her2 comparison between $\mathrm{CB}$ and $\mathrm{SB}$ did not yield significant results.

\begin{tabular}{|c|c|c|c|c|}
\hline & $\mathrm{CB}$ & $\mathrm{SB}$ & Discordant IHC & p value \\
\hline ER+ & 24 & 20 & 4 & 0.29 \\
\hline ER- & 3 & 7 & 4 & 0.29 \\
\hline PR+ & 15 & 13 & 2 & 0.78 \\
\hline PR- & 12 & 14 & 2 & 0.78 \\
\hline Her2 $(3+)$ & 2 & 2 & 0 & $>0.5$ \\
\hline Her2 (2+) & 5 & 2 & 3 & $>0.5$ \\
\hline $\operatorname{Her} 2(0 / 1+)$ & 20 & 23 & 3 & $>0.5$ \\
\hline
\end{tabular}

Conclusions: Formalin-fixed CB that are adequately cellular represent a better platform for IHC studies in patients with metastatic breast cancer to bone when compared to SB. The decal and crush artifact in SB can lead to delayed reporting and less accurate $\mathrm{IHC}$ results.

1962 Studying Amended Reports: Testing Effects of Time of Sign Out, Resident Involvement, and Specimen Type on Amendments O Alassi, R Varney, F Meier, R Zarbo. Henry Ford Health System, Detroit, MI.

Background: Amended reports may provide insights into conditions of practice that contribute to errors that lead to amendment.

Design: Among amended reports over 18 months. Amendments that revised primary diagnoses, revised secondary diagnostic information (tumor stage, grade, and margin), corrected patient mis identification (mis-ID) were examined. We also noted whether amendments were due to omitted diagnoses. We excluded amendments due to Mis-IDs undiscoverable at the time of sign-out. The reports were studied for date and time of original case signout, resident involvement and their level of training (junior,1st and 2 nd year) and senior (3rd and 4th year) and type of specimens whether it is biopsy or large specimen.

Results: The amendments on 9/64 (14\%) of examined reports had been initially misclassified: 8 designated 'diagnosis omitted' proved to be revised primary diagnoses, 1 'revised secondary diagnostic information' proved to be a revised primary diagnosis. After these corrections, among 64 amendments we found 16 primary revised diagnoses, 12 revisions of secondary diagnostic information (6 staging, 3 locations, and 3 laterality). 15 omitted diagnoses, and 15 corrected discoverable Mis-IDs. Almost three quarters $[47 / 64(73 \%)]$ of amendments were signed out in the afternoon, more than a third [23/64 (36\%)] after 3:00 PM. Residents were involved in only 9/64 (14\%) of amendments; senior residents were involved in 6 of these 9 cases. 13/16 (81\%) of primary revised diagnoses were biopsies. More than $9 / 10$ of omitted diagnoses were also biopsy specimens. The 15 discoverable misIDs were all biopsies. 7/12 (58\%) of revised secondary information, however, regarded large specimens.

Conclusions: First, [14\%] of amendments had been initially misclassified: better education of staff about amendment classification is necessary. Second, although we are working towards a continuous specimen flow, cases for sign-out accumulating in the afternoon; we hypothesize, increases staff fatigue and pressure to finalize biopsies within the expected two-day turnaround time: in the afternoon haste makes waste measured by amendments. Third, resident involvement did not appear to figure in amendments. Fourth, biopsies, in greater volume, nature of biopsy to reach an initial diagnosis, and great similarity from case to case, accounted for most primary diagnoses requiring revision, most of the omitted diagnoses, and most preventable misIDs. large specimens, on the other hand, with more secondary features, required more amendments for secondary diagnostic attributes.

1963 A Temporal Analysis of Trends in Intraoperative Consultation in a Large Academic Center

JA Bennett, M Walls, HS Crist, H Mani. Penn State Hershey Medical Center, Hershey, PA.

Background: Evaluation of trends in utilization of laboratory services is important for quality assurance, planning and education. We undertook a temporal analysis of the utilization of pathology intraoperative consultation (IOC) services in a large academic center, to look for changing trends, if any.

Design: The pathology database was searched to identify all cases that had IOC over two different periods (2002-2003 and 2010-2011). Results were tabulated by specialty, reason for consultation (diagnosis, margins, or both), deferral rates, and discordance, and trends were evaluated.

Results: Overall, IOC utilization remained comparable between the two periods analyzed $(4.2 \%$ of all surgical cases for $2002-2003$ and $3.6 \%$ for $2010-2011, \mathrm{p}$ 
$0.14)$. IOC increased significantly in $2010-2011$ for breast cases $(1.0 \%$ vs $8.6 \%$, $p$ $<0.01)$, due to increase in sentinel node evaluations. There was also an increase for pancreatohepatobiliary $(7.3 \%$ vs $12.3 \%, \mathrm{p} 0.07)$, genitourinary $(6.9 \%$ vs $10.6 \%$, p $0.11)$ and skin $(2.6 \%$ vs $5.6 \%, \mathrm{p} 0.18)$ cases. IOC decreased for gynecologic $(17.5 \%$ vs $2.4 \%$, p 0.04$)$ and neurological $(12.6 \%$ vs $8.0 \%$, p 0.05$)$ cases, and remained similar for pulmonary $(6.3 \%$ vs $8.7 \%$, p 0.31$)$, musculoskeletal $(16.1 \%$ vs $11.9 \%$, p $0.33)$, ENT $(18.1 \%$ vs $20.1 \%$, p 0.4$)$ and gastrointestinal $(7.2 \%$ vs $8.1 \%$, p 0.4$)$ cases. Although the primary reason for requesting IOC was for a diagnosis in both periods studied, the relative frequency of cases requiring diagnosis significantly decreased in $2010-2011$ ( $84 \%$ vs $67.2 \%, p 0.02$ ). This was due to an increase in number of cases requiring margin evaluation $(12.9 \%$ vs $26.6 \%, \mathrm{p} 0.04)$. The number of parts per case increased in 2010-2011 (1.7 vs $2.3, \mathrm{p}$ 0.05), with multi-part cases primarily seen in ENT and skin resections due to multiple margins requiring frozen sections. Margins were also requested more frequently in nephrectomies and esophagogastrectomies. The deferral rate was comparable $(9.4 \%$ vs $7.8 \%, \mathrm{p} 0.16)$ between the two periods, with similar specimens being deferred (thyroid follicular neoplasms, glial neoplasms, and lymphoid/spindle cell proliferations). The number of discordant diagnoses also remained comparable $(3.0 \%$ vs $4.8 \%$, p 0.26$)$.

Conclusions: Although the rate of IOC has remained relatively constant over the past 10 years, there are significant differences in specimen types, with margin evaluation becoming more frequent. These changes likely reflect changes in surgical management paradigms over the past decade. Similar studies at other institutions could provide a benchmark to help plan for resource allocation and training needs.

\section{Mitotic Rate Cut-Point Reproducibility in Gastrointestinal Stromal Tumor (GIST) Grading}

M Bonert, SA Mortuza, SS Raab. Memorial University of Newfoundland/Eastern Health, St. John's, Newfoundland and Labrador, Canada.

Background: Mitotic rate is an important histomorphologic predictor of tumor behavior that is routinely estimated and recorded in surgical pathology reports. Increasingly, mitotic counting procedures are being standardized, as it has been recognized as a source of variability. In gastrointestinal stromal tumors (GISTs), the sample area is standardized to $5 \mathrm{~mm}^{2}$ and 1 mitosis $/ \mathrm{mm}^{2}$ has been chosen as a cut-point for grading. The reproducibility of this clinically important cut-point is not known. In breast pathology, the binomial distribution has been used to examine the mitotic rate cut-points.

Design: Mitotic counting was modeled as a random sampling problem using the binomial distribution. Using this model, the sample standard deviation and 95 percent confidence interval (CI) were calculated to the nearest mitosis, and the mis-classification rates around the cut-point were calculated using the software GNU Octave (http://www. gnu.org/software/octave/).

Results: The standard deviation is 2-7 mitoses $/ 5 \mathrm{~mm}^{2}\left(0.4-1.4\right.$ mitoses $\left./ \mathrm{mm}^{2}\right)$, for the standardized mitotic cut-point (5 mitoses) and sampling area $\left(5 \mathrm{~mm}^{2}\right)$. Doubling the sampling area to $10 \mathrm{~mm}^{2}$ reduces the standard deviation to $6-13$ mitoses $/ 10 \mathrm{~mm}^{2}(0.6$ 1.3 mitoses $/ \mathrm{mm}^{2}$ ). If the sample area is $25 \mathrm{~mm}^{2}$, the standard deviation is $0.76-1.20$ mitoses $/ \mathrm{mm}^{2}$. The 95 percent confidence interval is $0-10$ mitoses for $5 \mathrm{~mm}^{2}(0-2$ mitoses/ $\left.\mathrm{mm}^{2}\right), 3-17$ mitoses for $10 \mathrm{~mm}^{2}\left(0.3-1.7 / \mathrm{mm}^{2}\right)$ and $15-35$ mitoses for $25 \mathrm{~mm}^{2}(0.60-1.40$ mitoses $\left./ \mathrm{mm}^{2}\right)$. The confidence interval decreases less with larger sample areas. With the standardized area $\left(5 \mathrm{~mm}^{2}\right)$, if the true mitotic rate is $2,3,4$, or 5 mitoses $/ 5 \mathrm{~mm}^{2}$, then $1.7 \%, 8.4 \%, 11.1 \%$, and $38.4 \%$ of the cases would be over-graded, respectively, by chance alone. On the other hand, if the true mitotic rate is $6,7,8$ or 9 mitoses $/ 5 \mathrm{~mm}^{2}$ for a $5 \mathrm{~mm}^{2}$ area, then $44.6 \%, 30.1 \%, 19.1 \%$ and $11.6 \%$ of cases would be under-graded, respectively, by chance alone.

Conclusions: The mitotic grade in general and for GISTs in particular is not very reproducible if the mitotic count falls within a standard deviation of the cut-point. We suggest that quantification of measurement variability, such as the standard deviation, be included in the mitotic rate estimation, to alert users of this information about its level of uncertainty. A triage approach with a low initial sample area could be used to identify cases in close proximity to the mitotic rate cut-point; in such cases, a larger area could be used to increase mitotic grade accuracy.

\section{Validation of Whole Slide Scanning for Use in Real-Time Clinical Frozen Section Consultation}

SH Bradshaw, E Belanger, MLamba, KT Mai, SJ Robertson, H Sekhon. Ottawa Hospital, Ottawa, ON, Canada.

Background: Digital slide scanning offers the posibility for a tertiary care hospital to provide an intra-operative consultation service to satellite hospitals without requiring a staff pathologist to be physically present at the satellite hospital. Before such a service could be offered, however, slide scanning would need to be validated to ensure that the additional step does not introduce error in diagnosis or unacceptable delay. The goal of this study is to assess diagnostic accuracy on digital vs glass slides.

Design: 42 frozen section cases selected to represent the range of specimens requiring intra-operative consultation at our institution were digitally scanned. The specimens were chosen with bias toward difficult consults to increase the chance of uncovering potential issues with the slide scanning process. The digital and glass slides were reviewed by four pathologists, with a 30 day washout period between reviews. The distribution of cases is summarized in table 1 .

Case Distribution
\begin{tabular}{|l|l|}
\hline Case Type & $\mid$ \\
\hline Margin, GI & 6 \\
\hline Margin, skin & 4 \\
\hline Margin, lung & 4 \\
\hline Margin, other & 2 \\
\hline Lymph node & 5 \\
\hline Neutrophil count & 4 \\
\hline liver met & 3 \\
\hline Dx, gyne & 4 \\
\hline Dx, other & 3 \\
\hline Bx, R/O malignancy & 3 \\
\hline Other & \\
\hline
\end{tabular}

Results: Table 2 shows all correct diagnoses, discrepancies, and major discrepancies for glass slides and digital images for all reviewers combined. The number of major errors was not significantly different between digital and glass slides. The total major and minor discrepancies trended toward better performance for the glass slides.

Comparison of errors between glass and digital slides

\begin{tabular}{|c|c|c|c|}
\hline 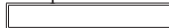 & Glass & Digital & $\mathbf{P}$ \\
\hline Correct diagnoses & 162 & 156 & \\
\hline Major errors & 4 & 3 & \\
\hline Total errors & 6 & 12 & 0.225 \\
\hline
\end{tabular}

Conclusions: Analysis of the individual discrepancies shows that reviewers of digital slides were more likely to defer to permanent section or give a diagnosis of 'suspicious' rather than committing to a positive or negative diagnosis, and this tendency led to a greater frequency of minor discrepancies. This may reflect a true degradation in the digitization of the slide, or alternatively, it may be at least partially due to unfamiliarity with the new media and reluctance to commit to a definitive diagnosis. Three out of four reviewers noted discomfort in couting neutrophils using digitally scanned images. Interestingly, despite this, there were no discrepancies (major or minor) relating to cases of neutrophil counts. Future work is necessary to assess if the number of minor discrepancies changes after the reviewers acquire familiarity with the new media. In addition, the time needed to arrive at a diagnosis using digital material relative to glass slides requires assessment.

1966 Inter- and Intra-Observer Agreement in Diagnosing Dysplasia in Barrett's Esophagus: Comparison of Routine Glass Slide vs. Digital Image Examination

SH Bradshaw, D Driman, MP Dupre, VG Falck, R Kirsch, A Meliti, B Nguyen, D Owen, A Rahemtulla, RH Riddell, H Sapp, G Soucy, C Streutker, EC Marginean. TOH, Ottawa, ON, Canada; St Luc, Montreal, QC, Canada; St. Michael's, Toronto, ON, Canada; LHSC, London, ON, Canada; Mount Sinai, Toronto, ON, Canada; CLS, Calgary, AB, Canada; SHR, Saskatoon, SK, Canada; VGH, Vancouver, BC, Canada; BCCA, Vancouver, BC, Canada; QEII HSC, Halifax, NS, Canada.

Background: Whole-slide imaging offers promise for Internet-based telepathology consultations between institutions and for use in daily sign out. The aim of this study is to investigate the inter- and intra-observer variability in diagnosis of Barrett's esophagus (BE) dysplasia, using digital images and glass slides.

Design: Sixty cases of BE with diagnoses including negative for dysplasia (NEG), low grade dysplasia (LGD), indefinite for dysplasia (IND), high grade dysplasia (HGD), intramucosal carcinoma (IMC) and invasive carcinoma (ICA) were scanned. The digital and glass slides were reviewed by 12 expert GI academic pathologists, with a 30 day washout period between reviews.

Results: Table 1 shows the inter-observer Kappa statistic using six diagnostic categories, and a simplified three category system: 1 NEG, 2 LGD/IND and 3 HGD/IMC/ICA. Table 2 shows the kappa statistic for the intra-observer variability when comparing glass slides vs. digital images. Overall, the inter-observer agreement is similar for glass and digital images, slightly better for glass slides. Agreement varies from slight to moderate using six categories, and improves to fair to substantial using three categories. The intra-observer agreement between glass and digital slides is similar to inter-observer agreement within the same media.

\begin{tabular}{|c|c|c|c|c|}
\hline $\begin{array}{l}\text { Inter-o } \\
\text { Dx }\end{array}$ & Glass (6) & Digital (6) & Glass (3) & $\mid \overline{\text { Digital (3) }}$ \\
\hline NEG & 0.54 & 0.48 & 0.54 & 0.48 \\
\hline IND & 0.15 & 0.16 & $0.37^{*}$ & $0.28^{*}$ \\
\hline LGD & 0.21 & 0.15 & & \\
\hline HGD & 0.47 & 0.40 & $0.74 * *$ & $0.68^{* *}$ \\
\hline IMC & 0.45 & 0.36 & & \\
\hline ICA & 0.39 & 0.43 & & \\
\hline Overall & 0.38 & 0.33 & 0.55 & 0.48 \\
\hline
\end{tabular}

${ }^{*}$ Combined IND/LGD; ${ }^{* *}$ Combined HGD/IMC/ICA

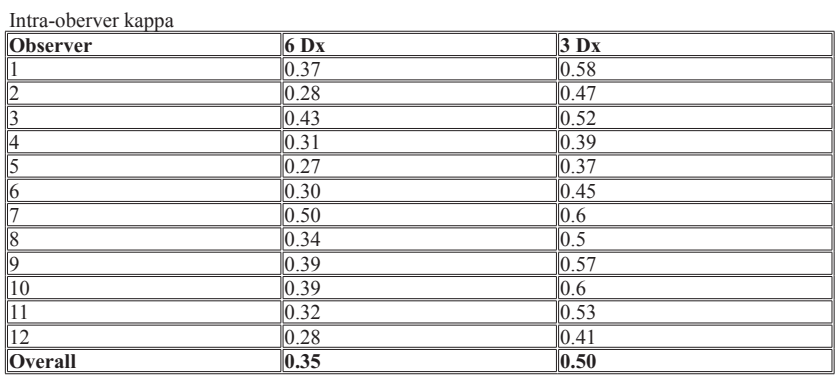


Conclusions: There is less inter-observer variability with three categories than with six. It is unclear whether the intra-observer variability between glass and scanned images is due to a change in media or simply the result of baseline intra-observer variability within the same media. Future work will evaluate the intra-observer variability with no change in media. The potential for digital slide diagnosis in clinical practice warrants further validation of this technology.

\section{Predictive Molecular Markers in Lung Cancer - 80\% Decrease} in Diagnosis to Treatment Decision Times by Streamlining EGFR and ALK Testing Protocols

M Cankovic, L Whiteley, S Michalowski, CH Stone, RJ Zarbo, DA Chitale. Henry Ford Hospital, Detroit, MI

Background: Lung cancer is one of the deadliest cancers and starting treatment soon after diagnosis can be critical to patient survival. Mutations in epidermal growth factor receptor (EGFR) and EML4/ALK are detected in $15 \%$ and $1 \%$ of patients with lung adenocarcinoma, respectively. Both mutations are now used as targets for new anticancer drugs. Molecular genotyping can immediately predict which patients are likely to respond as well as identify those who are likely to experience resistance.

Design: The aim was to perform trend analysis using a value stream map and determine turn around times (TATs) from patient's surgery/diagnosis to the time treatment decisions can be made (both EGFR and ALK results available). Study period lasted from March 1, 2011 to September 5, 2012, divided in 4 phases: Phase $1(3 / 1 / 11$ to 8/22/11) - no established pathway; lack of familiarity with process; lack of coordination; EGFR performed in AP Molecular Lab; ALK a send out test. Phase 2 (8/23/11 to 4/30/12) - ALK brought in house; Phase 3 (5/1/12 to 7/31/12) - increase system clinicians' awareness (consultations, tumor boards, internet resources); ALK becomes a reflex test when EGFR negative. Phase $4(8 / 1 / 12$ to $9 / 5 / 12)$ - test requesting process streamlined; specimen handoffs between AP Molecular Lab and Cytogenetics Lab redesigned.

Results: Diagnosis-to-treatment-decision turn around times (TATs): Phase $1=61$ days ( 6 cases); Phase $2=35$ days ( 25 cases); Phase $3=17$ days ( 56 cases); Phase $4=12$ days (24 cases)

$-80 \%$ decrease in time from surgery/diagnosis to start of treatment (shortened from 61 days to 12 days)

-Patients with a deadly cancer are treated much more quickly - increase in patients' quality of life and possibly overall survival.

Cost assessment:

$-43 \%$ decrease in handoffs ( 14 steps cut to 8 steps - \$200/case x 150 cases/year $=$ $\$ 30,000 /$ year saved)

-Outsourced testing brought in house (\$500/case x 130 EGFR negative cases/year = additional $\$ 65,000$ saved)

Conclusions: Value stream maps helped us to redesign processes, lower costs, and improve TATs. Clinicians' close communication with pathology staff ensures that testing is ordered in a timely manner. Paths for specimen handoffs and result reporting are well defined. By systematically focusing on the entire testing process we were able to decrease diagnosis to treatment decision times for lung adenocarcinomas from 61 to 12 days, eliminating $\$ 95,000$ /year of unnecessary expenses. This project necessitated coordinated efforts of different individuals across the system separated by geography, specialty and leadership structure.

1968 Impact of Different Antibody Clones on Ki-67 Labeling Index Assessment in Breast Carcinoma

BZ Clark, MM Desouki, DJDabbs, KMcManus, R Bhargava. Magee-Womens Hospital of University of Pittsburgh Medical Center, Pittsburgh, PA.

Background: Ki-67 proliferative index (LI) by immunohistochemistry is used in invasive breast carcinoma as a prognostic indicator, especially in estrogen receptorpositive breast carcinoma. Variability in Ki-67 LI may occur due to inter-observer variability, area of the tumor counted, and tumor heterogeneity. Although guidelines for interpreting $\mathrm{Ki}-67$ results have been published, the impact of various clones on Ki-67 LI is still not well documented.

Design: In this quality assurance study of 46 invasive breast carcinomas, we determined Ki-67 LI using three antibody clones (30-9, SP-6, and MIB-1) to Ki-67. All samples were routinely fixed for $8-48$ hours. In addition, we performed the staining with the same clones on paired tumor samples fixed for 96 hours to determine the impact of prolonged fixation on each clone. A single pathologist determined the Ki-67 LI for all clones and all cases, in order to minimize variability. The difference in Ki-67 LIs between different clones was determined using paired t-tests.

Results: Following routine fixation for 8-48 hours, the mean Ki-67 LI was highest for SP6 and lowest for MIB1 (mean Ki-67 LI of 36\% for SP6, 34\% for 30-9, and 30\% for MIB1; p-value 0.3700 for 30-9 and SP6, 0.0124 for 30-9 and MIB1, and 0.0002 for SP6 and MIB1). Following 96-hour formalin fixation, mean Ki-67 LI remained highes for SP6 and lowest for MIB1 (mean Ki-67 LI of 37\% for SP6, 30\% for 30-9, and 27\% for MIB1; $p$ value $<0.0001$ for 30-9 and SP6, 0.0006 for 30-9 and MIB1, and $<0.0001$ for SP6 and MIB1). For the 2 clones showing the largest difference among each other, SP6 showed $>10 \%$ age point higher Ki-67 LI than MIB1 in $18 \%$ of routinely fixed cases and in $39 \%$ of cases fixed for 96 hours.

Conclusions: The antibody clones for Ki-67 show statistically significant differences in mean LI, which could account for minor clinical differences in Ki-67 results from different institutions. The SP6 antibody clone was not affected by prolonged fixation and the 2 other clones (30-9 and MIB1) showed slight reduction in mean LI in the cases fixed for 96 hours. In a separate analysis, however, we found the difference (between routine fixation and 96 hours fixation) for these 2 clones to be clinically insignificant. The different clones used for Ki-67 analysis add to variability in LI, in addition to tumor heterogeneity, method of analysis, and inter-observer variability.
1969 Impact of 96-Hour Formalin-Fixation on Assessment of Ki-67 Labeling Index

BZ Clark, MM Desouki, DJ Dabbs, KMcManus, R Bhargava. Magee-Womens Hospital of University of Pittsburgh Medical Center, Pittsburgh, PA.

Background: Ki-67 labeling index (LI) by immunohistochemistry is used in invasive breast carcinoma as a prognostic indicator. ASCO/CAP guidelines recommend a duration of formalin fixation of 6-72 hours for hormone receptors but no upper limit of fixation is proposed for $\mathrm{Ki}-67$. We have previously shown that fixation up to 96 hours does not significantly impact hormone receptor status (Am J Clin Pathol. 2012;137:691-698). In this quality assurance study of 46 invasive breast cancer cases, we determined Ki-67 LI using three antibody clones to Ki-67 following both routine fixation (8-48 hours) and prolonged ( 96 hour) fixation.

Design: Forty-six surgical resections for invasive breast carcinoma with sufficien tissue for diagnosis were included. One tumor section was fixed in formalin for 96 hours. Immunohistochemical stains for three antibody clones to Ki-67 (30-9, SP-6, and MIB-1) were performed. Ki-67 LI was manually examined by one pathologist on all clones for all cases (both routinely fixed and fixed for 96 hours) to avoid inter-observer variability. Mean Ki-67 LIs for each antibody clone were compared to each other for routinely fixed tissue and fixed for 96 hours using paired t-test.

Results: Using the $30-9$ antibody clone to Ki-67, there was a statistically significant difference in mean Ki-67 PI for cases with routine fixation vs. 96 hours ( $\mathrm{p}=0.036$ ), while the duration of fixation did not affect the mean Ki-67 LI for antibody clones SP-6 $(\mathrm{p}=0.15)$ or MIB-1 $(\mathrm{p}=0.18)$. Although the differences with $30-9$ clone were statistically significant only 9 of 46 cases had $>10 \%$ age point difference. Moreover, 3 of these 9 cases actually had higher Ki-67 LI with 96 hours fixation. For SP6 antibody, 5 cases had $>10 \%$ age point difference, of which 4 cases showed higher Ki-67 LI in tumors fixed for 96 hours. For MIB1 antibody, 7 cases had $>10 \%$ age point difference, of which 1 case showed higher Ki-67 LI in tumors fixed for 96 hours.

Conclusions: A clinically significant difference was not observed in mean Ki-67 LIs with any of the antibody clones when invasive breast carcinomas were fixed for 96 hours in formalin. Our study validates performance of Ki-67 staining in tissue fixed for up to 96 hours.

1970 Efficacy and Cost of Using FibroSURETo Evaluate Hepatic Fibrosis in a Veterans Administration Patient Population: A Pilot Study

EF Clayton, D Kaplan, K-M Chang, J Sepulveda, L Chandler, N Jhala, D Jhala. Hospital of the University of Pennsylvania, Philadelphia, PA; Veterans Administration Medical Center, Philadelphia, PA.

Background: Liver biopsy is the gold standard for monitoring disease progression in patients with chronic liver disease. FibroSURE, a commercially available test which uses serum biomarkers to predict fibrosis and activity, is a non-invasive alternative to liver biopsy. We study the use of FibroSURE at a Veterans Administration Medical Center and assess its diagnostic efficacy and cost-effectiveness.

Design: We retrospectively identified patients who underwent both FibroSURE and liver biopsy at our institution in a one-year period. The original liver biopsy slides were independently re-reviewed by two pathologists and assigned METAVIR scores. METAVIR scores were then compared with the patients' FibroSURE results. The institutional costs per test were obtained.

Results: Of the 796 patients with FibroSURE results during the study period, 22 had concurrent FibroSURE testing and liver biopsy. Twenty biopsies were available for review. All 20 patients (19 males, 1 female, mean age 56.65 years, range 40-63 years) had a history of Hepatitis C. All 20 liver biopsies yielded diagnostic tissue; results below. METAVIR score determined by liver biopsy $(\mathrm{n}=20)$

\begin{tabular}{|l|l||l|l|}
\hline Fibrosis & Activity \\
\hline Stage & n (\%) & Stage & n (\%) \\
\hline F1 & $10(50)$ & A0 & $3(15)$ \\
\hline F2 & $7(35)$ & A1 & $7(35)$ \\
\hline F3 & $1(5)$ & A2 & $8(40)$ \\
\hline F4 & $2(10)$ & A3 & $2(10)$ \\
\hline
\end{tabular}

Two $(10 \%)$ of the 20 patients had non-diagnostic FibroSURE results. None of the patients had complete concordance between FibroSURE and liver biopsy for both fibrosis and activity scores. Relative performance of FibroSURE to liver biopsy is shown. Comparison of FibroSURE Results to Liver Biopsy

\begin{tabular}{|l|l|l|}
\hline \hline & Fibrosis & Activity \\
\hline Concur n(\%) & $3(17)$ & $10(55)$ \\
\hline Underestimate n(\%) & $2(11)$ & $3(17)$ \\
\hline Overestimate n(\%) & $13(72)$ & $5(28)$ \\
\hline
\end{tabular}

The institutional cost of liver biopsy was $\$ 289.43$, plus $\$ 65.99$ for pathology interpretation. The institutional cost of FibroSURE was $\$ 105$.

Conclusions: Preliminary comparisons with liver biopsy show FibroSURE may overestimate fibrosis and activity in patients with Hepatitis C, and suggest that in this population, FibroSURE is more accurate in assessing activity than fibrosis. Our study also reveals a greater than three-fold institutional cost difference between liver biopsy and FibroSURE. Expanding the study population and cost-benefit analysis will allow us to determine strategies for controlling health care costs while maintaining quality of care for Hepatitis $\mathrm{C}$ patients in our institution.

1971 A Rapid Improvemment Event Using Lean Concepts Can Substantially Improve Histology Laboratory Efficiency

JA Collins, N Grandhi, R Prasad, WS Twaddell, PN Staats. University of Maryland Medical Center, Baltimore, MD.

Background: Pathologist workflow relies on the ability of a histology lab to produce quality slides in a timely manner. Common obstacles labs need to overcome include understaffing, machine down times, repeat processing, physical distance and facility 
space, and interruptions. The Lean process was initially designed for manufacturing to decrease non-value added effort and improve efficiency. More recently, the medical field and the pathology community in particular has taken an interest in these techniques. Our medium-sized pathology lab ( [/underline]15,000 surgical specimens per year) recently undertook a "rapid improvement event" (RIE) focused on improving the efficiency of producing routine slides in the histology lab.

Design: The RIE was a week-long rapid, focused effort to improve productivity. The team included pathologists, residents, histology staff, and "fresh eyes" from other areas of pathology, working in a collaborative manner to recognize problems, identify their root causes, and rapidly implement experiments aimed at improvement. The RIE involved extensive adjustments to the physical space and layout, with immediate onsite help from facilities and bioengineering staff. A six sigma approach to eliminating waste and removing extraneous items from the work space was implemented. Severa measures of lab-wide productivity were collected before and after the RIE, with a focus on time of availability of finished slides.

Results: The RIE resulted in significant improvements in productivity, while also improving histology staff morale. There was a marked decrease in histology turn-aroundtime, from $64 \%$ of biopsy slides prepared by $11: 30 \mathrm{AM}$ to $100 \%$ by $10: 00 \mathrm{AM}$; and from $76 \%$ of routine slides prepared by $5 \mathrm{pm}$ to $100 \%$ by $5: 00 \mathrm{pm}$. The physical space utilized to produce histological slides for routine (hematoxylin and eosin staining), specia stains and immunohistochemical stains decreased by $34 \%$ from $2208 \mathrm{ft}^{2}$ to $1466 \mathrm{ft}^{2}$

Conclusions: A RIE using Lean concepts resulted in rapid and sustained improvement of slide preparation time due to optimization of work-flow, while improving staff morale and maintaining slide quality. The reduced time spent on non-essential activities by cutters and embedders appeared to be the most important element in increasing efficiency. A collaborative approach, with pathologists, managers, and histotechnologists is critical. The basic principles of "Lean thinking" are commonly utilized for commercial applications, but have clear implications for identifying waste and improving work efficiency within the context of a university hospital histology laboratory.

\section{Lean Redesign of Operating Room Specimen Labeling Process} $R$ DAngelo, $S$ Ruediger, $R$ Varney, A Munkarah, RJ Zarbo. Henry Ford Hospital, Detroit, MI.

Background: Specimen safety results from processes initiated by Surgery and then owned by Pathology. Defects commonly exist in the processes of specimen collection, labeling and hand off from the Operating Room (OR) to the Pathology Laboratory (Lab). Correction of these problems requires aligned standard work and effective communication between both departments.

Design: In an attempt to redesign this process, 3 cross-departmental teams at Henry Ford Hospital, Detroit, consisting of gyn-oncology surgeons, OR nurses, scrub techs, pathologists, and quality staff reviewed surgical processes for specimens from OR to Lab. Teams were charged with identifying and minimizing variation in order to adopt by consensus one pathway to collect, label and deliver Gyn Onc specimens to Lab. Teams directly observed surgeries to observe current OR specimen related processes, then created a value stream map depicting waste, redundancy and potential patient safety issues. Each team met weekly, instructed to develop the "perfect process" that was then presented for team selection of best standardized process. We describe design of a waste-free, error-proof approach to collect and label specimens in absence of computer generated labels.

Results: The most significant patient safety related event was OR handwritten patent labels with specimen identification. A system of handwritten labels without tracking forced nurses to document by hand the same label and container information up to 10 times for each case. For tracking, nurses kept a personal specimen tally then documented this information all over again in the Lab specimen receipt log. As a result of the multidepartmental effort a standardized approach enlisting a direct connection was created between surgeon, scrub nurse, circulator and Lab. To remove redundant handwriting, a specimen labeling packet was innovated that incorporated custom designed carbonless piggy-back labels to document the multiple copies by writing specimen and patien information only once on each lab requisition. Lab requisitions were revised to accept these first-time labels making secondarily written specimen information unnecessary. In this redesign, OR labeling and transfer steps were reduced by $45 \%$ (11 reduced to 6 steps). Further upstream, patient information labels generated upon development of patient charts in preparation for surgery, could now be used instead of hand relabeling each specimen.

Conclusions: We have demonstrated the power of multi-disciplinary teams applying Lean at the level of work to focus on patient safety and efficiency by mutually redesigning processes that meet the requirements of both OR and Laboratory.

\section{Pragmatic Algorithm for Improving the Diagnostic Quality of Fine Needle Aspiration Cytopathology in the Classification of Hematolymphoid Proliferations}

BH Durham, A Afify, H Jess, JY Song, DM Dwyre, M Chen. University of California, Davis, School of Medicine, Sacramento, CA.

Background: Fine needle aspiration (FNA) cytopathology is a common diagnostic screening tool for suspected hematolymphoid malignancies. With an atypical lymphoid proliferation in the differential diagnosis, flow cytometric analysis is implemented. However, after preparation of the routine cytopathology smears and the cell blocks, sufficient material is sometimes unavailable for complete flow cytometric analysis. Therefore, the application of a selected panel of immunohistochemistry (IHC) with supplemental molecular studies on the FNA cell blocks can be meaningful in developing a timely and precise classification of these entities.

Design: We retrospectively reviewed the laboratory information system for FNAs performed from 2002-2012 at an academic medical center where a lymphoproliferative disorder entered into the differential diagnosis. The 240 FNAs are categorized into those that are positive for malignancy, atypical lymphoid proliferation-suspicious for malignancy, reactive, granulomatous, or nondiagnostic. A subset of 30/240 FNAs have a corresponding hematopathological tissue diagnosis and a cell block with sufficient material for additional testing. We have designed a feasible and tactical IHC diagnostic algorithmic panel (CD3, CD5, CD10, CD20, CD30, CD56, CD79a, CD138, Cyclin D1, MUM-1, and Ki-67) to classify the common hematolymphoid proliferations from cell blocks. The addition of CD56, Cyclin D1, and MUM-1 is to help differentiate a plasma cell neoplasm from a B-cell lymphoma with plasmacytic differentiation. Additionally, we use cell block molecular testing in selected cases to refine the diagnoses.

Results: Of the reviewed 240 FNA cases, $42 \%$ are positive for malignancy; $35 \%$ are atypical lymphoid proliferations-suspicious for malignancy; $19 \%$ are reactive; $1 \%$ are granulomatous; and 3\% are non-diagnostic. The cell blocks of the selected subset of 30 FNA cases are evaluated by our IHC panel with the use of FISH and molecular studies in selected cases, which leads to a cell block-based classification with reasonable correlation $(70 \%)$ with the corresponding tissue diagnoses.

Conclusions: Therefore, we are proposing a pragmatic diagnostic algorithm for the classification of hematolymphoid proliferations using cell blocks. Thus, use of our IHC panel and molecular studies on cell blocks can be meaningful in generating more specific diagnoses prior to the acquisition of a definitive biopsy, which is a practical approach to hematolymphoid cytopathology for improving diagnostic patient care.

1974 Validation of Immunohistochemistry for Identification of IDH1 Mutation in Gliomas

CM Egoavil, A Paya, E Irles, A Garcia-Martinez, JL Soto, A Castillejo, MI Castillejo, T Quintanar, J Herrero, A Rodriguez-Lescure, MJ Portugues, P Moreno, L Heredia, $E$ Andrada, I Aranda, VM Barbera, C Alenda. University Hospital (HGUA), Alicante, Spain; University Hospital (HGUE), Elche, Alicante, Spain; (HGUE), Elche, Alicante, Spain; (HGUA), Alicante, Spain.

Background: Isocitrate Dehydrogenase 1 (IDHI) mutations occur in $60-90 \%$ of diffuse astrocytoma and secondary glioblastomas. $I D H I$ mutation is a relevant marker for diagnosis and prognosis of primary brain tumors. Immunohistochemical (IHC) assessment of this mutation might be a useful and accessible tool to distinguish between neoplastic and gliosis lesions. The aim of this study was the validation of an IHC process in formalin-fixed, paraffin-embedded brain tumor tissue to determine IDHI mutational status.

Design: A tissue microarray (TMA) built with 171 resected surgical gliomas specimens from Biobank HGUA and HGUE (14 stage I astroytoma; 22 stage II; 20 stage III and 114 glioblatomas) was analyzed by IHC with the mouse monoclonal Anti-Human IDH 1 (R132H Clone H09, Histonova). The TMA was examined by two pathologists. IHC was scored as negative (no staining), positive (cytoplasmic and nuclei staining in $>10 \%$ of tumor cells) and not evaluable cases. DNA sequencing was used as the gold standard technique to establish the $I D H 1$ mutational status.

Results: Results were available on 155 and 126 cases for IHC and DNA sequencing, respectively. Overall inter-observer agreement was high (weighted $\kappa=0.94$, SE: 0.043 CI $0.91-1$ ), with $98.7 \%$ concordance $(94.9-99.8 \%$ ). All concordant negative cases were $I D H I$ wild type $(\mathrm{n}=105)$, and all concordant positive cases were $I D H I$ mutated $(\mathrm{n}=21)$ [Sensibility: $100 \%$; specificity: $100 \%$; $\mathrm{p}=0$; statistical power: $>95 \%$ ].

Conclusions: Our study shows that evaluation of IDH1 by immunohistochemistry is highly reproducible with the application of standard immunohistochemical techniques and simple scoring criteria.

1975 The Difference between What Is Said and What Is Heard: Phrases of Uncertainty in Surgical Pathology Reports

CJ Ellermeier, J Fava, A Amin. Rhode Island Hospital, Providence, RI.

Background: The most common communication route between pathologists and clinicians is by surgical pathology report. As in many facets of medicine, surgical pathology diagnoses are not always definitive; therefore pathologists often use modifying phrases to reveal uncertainty. Studies have shown that clinicians often do not interpret pathology reports like pathologists intend. Such unintentional miscommunications could potentially cause significant medical errors.

Design: After obtaining IRB approval, a study was designed in two phases. In the first phase, an online survey was distributed nationally among pathologists with variable experience levels who were asked about their interpretation of 24 phrases using a five point scale with five representing highest certainty. The second phase of the study is to distribute a national survey for clinicians including questions carried over from the first phase. The goal of the survey is to determine and compare the level of certainty of modifying phrases among pathologists and clinicians of different subspecialties to recommend potential interventions to remedy such pitfalls.

Results: To date, 52 participants have responded to the pathologist-centered survey. Table 1 shows experience levels and types of appointments for the participants. Thus far the phrases that generate most certainty are "diagnostic of" and "represents" ( $86.3 \%$ $(\mathrm{n}=44)$ and $53.1 \%(\mathrm{n}=26)$, respectively), while "questionable" and "equivocal" create the most uncertainty $(38.8 \%(\mathrm{n}=19)$ and $32 \%(\mathrm{n}=16)$, respectively). $63.5 \%$ of participants believed that additional communications like interdepartmental meetings were often useful in resolving misinterpretations. The clinician-centered survey is currently distributed and awaiting data collection.

Table 1- distribution of participants

\begin{tabular}{|l|l||}
\hline A-Training level & $\%$ \\
\hline In training & $34.6(\mathrm{n}=18)$ \\
\hline In practice & $65.4(\mathrm{n}=34)$ \\
\hline B-Practice type & \\
\hline Academic & $84.6 \%(\mathrm{n}=44)$ \\
\hline Community based & $11.5 \%(\mathrm{n}=6)$ \\
\hline Industry/Government & $3.9 \%(\mathrm{n}=2)$ \\
\hline
\end{tabular}


Conclusions: Surgical pathology reports are the major conduit of information distribution with word choice playing a very important role. This will be the first study to assess interpretation of surgical pathology reports by multiple clinical specialists. Ambiguous phrases (i.e. "suggestive of" or "suspicious for") may not be similarly interpreted by pathologists and clinicians, and deserve further investigation to elucidate unintentional, but potentially serious, miscommunications. It may be useful to recommend standard phrases to help reduce the misunderstandings.

1976 Touch Preparations for Intraoperative Diagnosis of Sentinel Lymph Nodes after Neoadjuvant Therapy for Breast Cancer Have a High False Negative Rate

RM Elliott, RR Shenk, HL Gilmore. University Hospitals Case Medical Center, Case Western Reserve University, Cleveland, $\mathrm{OH}$.

Background: Intraoperative analysis of sentinel lymph nodes (SLNs) for breast cancer has been a common request by surgeons over the past decade because an axillary lymph node dissection (ALND) was done at the time of surgery in patients with a positive result. Touch preparation (TP) has become a preferred method by many pathologists because of reported high sensitivities and rapid turnaround time. However, prior studies looking at the sensitivity and specificity of TP for SLN diagnosis have not been performed on patients who have had prior therapy. There is increasing data that the use of SLN biopsy in patients after neoadjuvant therapy is accurate and results in the sparing of the axilla for a significant number of patients. However, after treatment, many of these SLN have significant treatment changes including extensive fibrosis that may impact the intraoperative assessment by TP for diagnosis.

Design: Surgical pathology records from 2011 were searched for all intraoperative evaluations of SLNs by TP on patients who had received neoadjuvant therapy. The results from the intraoperative diagnosis were compared with the final pathology reports to determine the rate of concordance and discordance.

Results: Overall, there were a total of 58 sentinel lymph nodes from 23 different patients that were evaluated by TP intraoperatively. Of the 51 SLNs that were negative intraoperatively by TP, $12(24 \%)$ were actually positive on permanent sections. There was 1 SLN that had suspicious cells that was positive on permanents. All 6 SLNs that were positive on TP were also positive on permanents. Overall, 6 of 23 patients $(26 \%)$ had at least one discordant result. Notably, of the 18 patients who had negative SLNs by TP intraoperatively, 10 went on to have an ALND at the time of surgery despite the negative result, and 1 patient with a positive review at the time of surgery did not go on to ALND.

Conclusions: The use of TP for the diagnosis of malignancy in SLNs after neoadjuvant therapy has significant limitations and results in a high false negative rate. Intraoperative diagnosis of SLN should be used only in those cases where a positive result truly will determine the need for ALND at the time of surgery, and additional sampling by frozen section should be considered to improve the accuracy of intraoperative diagnosis.

1977 The International Collaboration on Cancer Reporting (ICCR): Development of Evidence Based Core Data Sets for Pathology Cancer Reporting

DW Ellis, J Dvorak, L Hirschowitz, M Judge, A Kwiatowski, J Srigley, KK Washington, $M$ Wells. Royal College of Pathologists of Australasia, Sydney, NSW, Australia; College of American Pathologists, Northfield, IL; Royal College of Pathologists, London, United Kingdom; Canadian Partnership Against Cancer, Toronto, Canada; Canadian Association of Pathology, Toronto, Canada.

Background: Cancer pathology data sets (CPDS) are foundational elements for clinical cancer care. Global collaboration and standardization of CPDS is essential for international benchmarking and epidemiological research in cancer and to reduce the burden of dataset production yet this has not been previously attempted.

Design: A quadripartite group, the International Collaboration on Cancer Reporting (ICCR), including Pathology Colleges and Associations of the USA, UK, Canada and Australia and representing a population of approximately 450 million, was established in 2011 to examine the practicability of developing common, internationally agreed and evidence-based CPDS. As a pilot, the ICCR established 4 international review panels (RP) to develop CPDS for prostate, lung, endometrial carcinoma and melanoma, each comprising 9 physicians: an ICCR pathology lead and 2 internationally renowned expert representatives from each country. Existing CPDS from each country were collated and each chair facilitated an evidence-based review and harmonization of the core (required) data elements, permitted responses, non-core (recommended) elements and terminology. Core elements included stage, tumour type and predictive or prognostic data for which there was Level III-2 evidence to support inclusion (BMC Med Res Method 2009, 9:34). Results: All 4 RPs completed their objectives within the allotted 4 months. Paradoxically, agreement between international experts proved simpler than in institutional or national programs. Through stringent criteria the RPs were able to remove legacy data elements and to rationalize a total of 118 pre-existing core elements to 66 (endometrium 23/17, prostate 38/18, melanoma 38/20 and lung 19/11). Definitions and permitted responses for all data elements were agreed.

Conclusions: This ICCR pilot study demonstrated that internationally standardized and evidence-based CPDS can be produced efficiently with significant improvements in content, including harmonized terminology, definitions and required responses. An operational plan to extend this standardization process to other languages, jurisdictions and cancer types is fundamental to improving cancer reporting globally and thereby international research and comparisons of cancer management.
1978 Repeat of Estrogen Receptor on Breast Resection Specimens with "Low ER" on Core Biopsy - A Quality Assurance Study

NC Gloyeske, DJ Dabbs, A Mallon, K McManus, R Bhargava. Magee-Womens Hospital of UPMC, Pittsburgh, PA.

Background: In breast cancer, the level of estrogen receptor (ER) expression as determined by immunohistochemistry (IHC) is an important prognostic and predictive marker. Therefore, the ASCO/CAP guidelines on hormone receptor testing suggest reporting ER IHC results semi-quantitatively. Usually hormone receptor studies are performed on the core biopsy specimen and if ER is positive ( $\mathrm{H}$-score $\geq 1$ ), the testing is not repeated on the resection. However, there is some concern if semi-quantitative results for "low ER positive cases" on core biopsy are accurate and representative of the entire tumor.

Design: We selected patients that had breast cancers with a low level of ER expression (H-score 1-100) on core biopsies performed in 2010. We compared the core biopsy result on each case with repeat ER H-score on one tumor block from the resection. The cold ischemic time was calculated for each of the resection specimens when it was available. Results: Of the 731 total ER positive breast cancers in 2010, 53 (7.3\%) cases had $\mathrm{H}$-scores of 1-100. Of these 53 cases, 37 had paired core biopsy and resection specimens containing invasive tumor which formed the basis of this quality assurance study. The mean $\mathrm{H}$-score for the core biopsies was 28 , and 18 for the resection $(\mathrm{p}=0.1252)$. The $\mathrm{H}$-scores on repeat testing ranged from $0-100$ with 8 of 37 cases becoming negative (i.e. H-score of 0). Eight patients received neoadjuvant therapy of which $4(50 \%)$ became negative on repeat testing. However only 4 of $29(14 \%)$ cases not treated with neoadjuvant became negative $(\mathrm{p}=0.0487)$. These 8 patients had similar H-scores on core biopsy in both groups (mean H-score of 26 in 4 tumors treated with neoadjuvant, and mean $\mathrm{H}$-score of 26.3 in 4 cases not treated with neoadjuvant). The most significant difference between tumors that became negative versus those that remained positive was the prolonged cold ischemic time in the former group. The mean cold ischemic time for tumors that became negative was 129 minutes (range: 20-337 minutes) compared to 52 minutes (range: 17-179 minutes) for tumors that remained positive $(\mathrm{p}=0.0078)$. Conclusions: Breast cancers expressing ER at a low level constitute a minority of ER positive cases. Core biopsies are better suited than resection specimens for determining low level ER expression because of negligible cold ischemic time. Although the difference in clinical behavior for low ER expressing tumors versus ER negative tumors is sometimes debatable, this study reiterates the importance of maintaining a cold ischemic time of one hour or less for IHC result accuracy.

1979 'Dot the Form' - An Easy Way To Improve Inpatient Biopsy Turnaround Time

X Hao, M Abbuhl, JE Willis. University Hospitals Case Medical Center, Cleveland, $\mathrm{OH}$; Case Western Reserve University, Cleveland, $\mathrm{OH}$.

Background: Inpatients have higher acuity illnesses for which rapid diagnoses are often imperative. Surgical pathology biopsies are often integral to this process in either the initiation or continuation [or withdrawal] of therapies. Efficient turnaround times (TAT) of biopsies is particularly important for meeting the needs of these patients in a timely manner. This study investigated whether a simple change to specimen processing would improve inpatient biopsy TATs in a busy academic medical center.

Design: All inpatient biopsies were identified at accessioning and the requisition annotated with a simple decal. In the histology lab, all requisitions with decals were identified and a second decal was placed on the specimen working draft. This allowed for the ready identification of inpatient biopsies by residents and staff. These cases received first priority for sign out throughout the day. This laboratory had previously implemented continuous flow specimen processing. TATs from date of accession to date of sign-out for all inpatient biopsies for two six month periods were calculated. 676 inpatient GI biopsy specimens from January to June of 2012 were prioritized using the above method. TAT time was compared to that of 792 inpatient GI biopsy specimens from January to June of 2010 signed out without prioritization. Data were analyzed using one sided $\mathrm{T}$ test.

Results: Average TAT improved for all routine and complex GI inpatient biopsies by $13 \%$ from an average of 3.4 days in 2010 to 3 days in $2012(p=0.02)$. For all routine inpatient GI biopsies, average TAT improved by $14 \%$ from 2.2 days in 2010 to 1.9 days in $2012(\mathrm{p}=0.01)$. There was no statistical difference of TAT between 2010 and 2012 for complex inpatient biopsies ( 2.8 days versus 3 days, $\mathrm{p}=0.13$ ).

Conclusions: A simple prioritization technique significantly improved all inpatient biopsy TATs allowing for early reporting of patient results. Given the complex variables involved, it is difficult to quantify the effects of improved inpatient biopsy TAT on overall patient stay. However since daily inpatient hospital stay costs in the US range from approx. $\$ 2700$ to $\$ 4500$ any process that improves inpatient biopsy TAT is likely to have significant cost savings. Institution of a simple triage and prioritization process for inpatient biopsies could potentially impact large numbers of patients and save significant healthcare resources without imposing any increased burden to Pathology Departments.

\section{A Comparisson of the Bethesda Thyroid Criteria: Academic} and Community Hospital Experiences

J Hertel, S Wobker, K Volmer, K Nance, S Maygarden. University of North Carolina at Chapel Hill, Chapel Hill, NC; Rex Healthcare, Raleigh, NC.

Background: In 2008 the Bethesda criteria were introduced to standardize the nomenclature for the interpretation and reporting of thyroid fine needle aspirations (FNA). Since its introduction, the Bethesda criteria have gained wide spread acceptance. While its use has standardized the terminology for reporting thyroid FNAs, the application, specifically, the use of atypia of undetermined significance (AUS) has 
been controversial and reported rates and usage vary. As a quality control measure we compared retrospective data from the initial use of the Bethesda Criteria and malignancy rates at a tertiary academic center and an affiliated community practice.

Design: The electronic databases for each institution were searched for all fine needle aspirates of the thyroid since the Bethesda criteria were adopted at the respective institution. Rates for each Bethesda category were determined for each institution and all cases were correlated with subsequent surgical resections if performed. Statistical analysis comparing the rates of Bethesda category usage and malignancy rates was performed using a Z-test for proportions.

Results: A total of 1038 thyroid FNA's were performed at the community hospital and 997 were performed at the academic medical center. The rates of use for all Bethesda categories except category VI (positive for malignancy) showed statistical significance. The most notable absolute differences were in the use of AUS significance and benign thyroid nodule (BTN). The community practice demonstrated a significantly lower rate of AUS $(2.7 \%$ versus $14.2 \%, \mathrm{p}<0.05)$ with a significantly higher rate of BTN $(75.4 \%$ versus $63.6 \%, p<0.05)$. Despite the significantly higher rates of BTN at the community practice, there were more surgeries $(\mathrm{p}<0.05)$ with a trend lower malignancy rate at the community hospital $(\mathrm{p}<0.10)$ on cases with a diagnosis of BTN. The higher use of AUS at the academic center resulted in a lower rate of surgeries for cases with a diagnosis of AUS

Conclusions: While there was, a higher rate of BTN use in the community setting there was no increased malignancy rate in the corresponding resections, implying that more liberal use of AUS at the academic center may not help identify additional malignant cases. However, it was also noted that there were significantly more surgical resections in the BTN category at the community hospital, indicating that a more conservative use of the AUS category may not reduce the number of surgical excisions.

\section{Crossing Boundaries: A Comprehensive Survey of Medica Licensing Laws and Guidelines Regulating the Interstate Practice of Pathology}

MC Hiemenz, ST Leung, JY Park. UT Southwestern Medical Center, Dallas, TX; Metropolitan Medical Laboratory, PLC, Davenport, IA; Children's Medical Center, Dallas, TX

Background: There are few specific guidelines or publications that address the interstate practice of pathology. Understanding the complexity of interstate licensure is critical for consultant pathologists as well as pathologists practicing in laboratories serving patients in multiple states. This is a timely topic because of recent malpractice and criminal judgments against pathologists involving interstate licensure issues.

Design: We performed a survey of the medical boards of all 50 states. For all 50 states we examined state medical practice acts, state medical board websites, and contacted each medical board for information regarding specific legislation or guidelines related to the interstate pathology practice. Based on this information we grouped states based on similarities in legislation and medical board regulations.

Results: The practice of medicine for pathology is based on the location where the specimen is obtained. The majority of states $(n=30)$ allow for limited out of state consultation with a physician licensed to practice in the specific state although numerous restrictions were identified. A minority of states $(n=6)$ allow for the limited practice of medicine without consultation but require the out-of-state physician to be temporarily present within that state when practicing medicine. Several states $(n=5)$ prohibit physicians from consultation without a license for the specific state; exceptions including the military and emergencies. This category of prohibition of consultation includes South Carolina which was one of the few states to specifically describe the role of the pathologist in the interpretation of laboratory data.

Conclusions: Increasingly the primary or consultative practice of pathology reaches across multiple state boundaries. Licensure requirements among states are heterogeneous and pathology practitioners of both anatomic and clinical pathology need to become proficient in the legal requirements governing the sending and receiving of specimens and consultative material. The legal risk lies with both pathologists that provide the primary interpretation to surgical specimens as well as consultants receiving cases in consultation. Furthermore, there is risk for pathologists interpreting not only surgical specimens, but also emerging esoteric tests (e.g., mutation analysis, FISH, flow cytomerty, genomics). Ignorantia legis neminem excusat - "Ignorance of the law excuses no one".

1982 A Single Institutional Experience of Frozen Section Practice in a Subspeciality-Based Setting - Should the Service Be Subspecialized? AP Hoschar, A Inman, DT Patil. Cleveland Clinic, Cleveland, OH.

Background: An important aspect of Quality Assurance in Anatomic Pathology is Frozen Section (FS) discrepancy analysis. The current study was undertaken to analyze the subspecialty FS discrepancy rates in a large volume practice and compare the rates between FSs interpreted with (cosigned) and without subspecialty (non-cosigned) expertise/input.

Design: A total of 8,726 FSs from June 2011-May 2012 were analyzed according to Association of Directors of Anatomic and Surgical Pathology guidelines (ADASP) criteria. The reasons for discrepancy were categorized into sampling and interpretation errors.

Results: Gross diagnosis was performed on $17(0.2 \%)$ and deferred on $100(1.2 \%) \mathrm{FSs}$. Of the remaining 8,609 FSs, there were $107(1.24 \%)$ discordant cases with 29 major $(0.34 \%)$ and $78(0.9 \%)$ minor discrepancies (See Table 1$)$.

\begin{tabular}{|c|c|c|c|c|c|c|c|}
\hline $\begin{array}{l}\text { Subspeciality (Total } \\
\text { FSs }=8609 \text { ) }\end{array}$ & \begin{tabular}{|l} 
Discre- \\
pancy \\
Major
\end{tabular} & \begin{tabular}{|l} 
Discre- \\
pancy \\
Minor \\
$\%)$
\end{tabular} & \begin{tabular}{|l||} 
Major \\
Interpre- \\
tation Discre- \\
pancies \\
$(\%)$ \\
$\%)$
\end{tabular} & \begin{tabular}{|l} 
Major \\
Sampling \\
Discre- \\
pancies \\
$(\%)$
\end{tabular} & \begin{tabular}{|l} 
Cosigned \\
FSs (n=517)
\end{tabular} & \begin{tabular}{|l|} 
Major \\
Cosigned \\
Discre- \\
pancies
\end{tabular} & \begin{tabular}{|l} 
Major \\
Non- \\
cosigned \\
Discre- \\
pancies
\end{tabular} \\
\hline ENT (2686) & $16(0.6 \%)$ & $27(1 \%)$ & $3(0.1 \%)$ & $13(0.5 \%)$ & $166(6.2 \%)$ & $\begin{array}{l}1(0.6 \%) \text { - } \\
\text { Sampling }\end{array}$ & \begin{tabular}{|l}
$15(0.6 \%)$ \\
{$[3-I n t e r p r e-$} \\
tation, \\
12 -Sampling $]$
\end{tabular} \\
\hline Pulmonary (1154) & 0 & $1(0.1 \%)$ & 0 & 0 & $51(4.4 \%)$ & 0 & 0 \\
\hline Orthopedic (927) & 0 & $3(0.3 \%)$ & 0 & 0 & $36(3.9 \%)$ & 0 & 0 \\
\hline Genitourinary (820) & $2(0.2 \%)$ & $8(0.9 \%)$ & 0 & $2(0.2 \%)$ & $49(5.9 \%)$ & 0 & $\begin{array}{l}2(0.3 \%)- \\
\text { Sampling }\end{array}$ \\
\hline Gynecology (642) & $1(0.2 \%)$ & $5(0.8 \%)$ & $\mid 1(0.2 \%)$ & 0 & $54(8.4 \%)$ & 0 & $\begin{array}{l}\text { Interpre- } \\
\text { tation }\end{array}$ \\
\hline Breast (635) & $7(1.1 \%)$ & $15(2.3 \%)$ & 0 & $7(1.1 \%)$ & $42(6.6 \%)$ & 0 & $\begin{array}{l}7(1.2 \%)- \\
\text { Sampling }\end{array}$ \\
\hline Neuropathology (580) & 0 & $4(0.7 \%)$ & 0 & 0 & $7(1.2 \%)$ & 0 & 0 \\
\hline Hepatobiliary (421) & $2(0.5 \%)$ & $7(1.7 \%)$ & $2(0.5 \%)$ & 0 & $38(9 \%)$ & $\begin{array}{l}1(2.6 \%)- \\
\text { Interpre- } \\
\text { tation }\end{array}$ & $\mid$\begin{tabular}{|l}
$1(0.3 \%)-$ \\
Interpre- \\
tation
\end{tabular} \\
\hline Gastrointestinal (341) & 0 & $3(0.9 \%)$ & 0 & 0 & $31(9 \%)$ & 0 & 0 \\
\hline $\begin{array}{l}\text { Dermatopathology } \\
(136)\end{array}$ & $1(0.7 \%)$ & $4(2.0 \%)$ & 0 & $1(0.7 \%)$ & $12(8.8 \%)$ & $\begin{array}{l}1(8.3 \%)- \\
\text { Sampling }\end{array}$ & 0 \\
\hline $\begin{array}{l}\text { Hematopathology } \\
\text { (116) }\end{array}$ & 0 & 0 & 0 & 0 & $5(4.3 \%)$ & 0 & 0 \\
\hline Soft tissue (110) & 0 & $1(0.9 \%)$ & 0 & 0 & $1(0.9 \%)$ & 0 & 0 \\
\hline Eye (33) & 0 & 0 & 0 & 0 & 0 & 0 & 0 \\
\hline Cardiac (18) & 0 & 0 & 0 & 0 & 0 & 0 & 0 \\
\hline
\end{tabular}

A total of 517 (6\%) FSs were cosigned with the corresponding subspecialty pathologist In specialities with same or slightly higher discrepancy rates in the non-cosigned group compared to the cosigned group, there were 4 interpretation errors (3- ENT, 1 -gynecology). Hepatobiliary pathology was the only speciality where the discrepancy rate was higher in the cosigned group due to an interpretation error $(\mathrm{n}=1)$.

Conclusions: The discrepancy rates in subspecialty practice setting are well within acceptable standards. Most discrepancies are due to sampling errors. As interpretation errors without subspecialty expertise are rare, FS practice may not require a dedicated subspecialized service.

\section{Should All Endometrial Tumors Be Tested for MSI by IHC? A} Pilot Analysis

A Kanbour-Shakir, DJ Dabbs, R Bhargava, E Elishaev. Magee-Womens Hospital of UPMC, Pittsburgh, PA.

Background: The lifetime risk for Human Non-Polyposis Colorectal Carcinoma (HNPCC) syndrome or Lynch Syndrome patients to develop endometrial cancer is $50-60 \%$ as compared to $2-3 \%$ in the general population. Microsatellite instability (MSI) is present in nearly all HNPCC patients and can be identified using polymerase chain reaction (PCR) assay. Immunohistochemical (IHC) staining for mismatch repair (MMR) protein expression (MLH1, MSH2, PMS2 and MSH6) provides similar information. Tumors with MSI or MMR protein deficiency have better prognosis and may respond differently to chemotherapy.

Design: We compared the rate of identifying possible Lynch Syndrome patients for 2 different time periods. Group 1 (February to July 2012): reflex MMR protein testing via IHC on patients 60 years and younger with non-serous morphology. Group 2 (August and September 2012): IHC reflex testing on all patients with endometrial carcinomas regardless of age and morphology, in order to identify all Lynch syndrome patients. PCR reflex testing for MSI were performed on all cases with indeterminate staining pattern by IHC. Cases were classified as showing MMR loss if they showed loss of protein on IHC or showed high MSI on PCR. The results from these 2 time periods were compared in this quality assurance study.

Results: A total of 42 cases were tested, $9(21 \%)$ showed MMR protein loss. Twentysix cases were tested prior to August 2012 with average of 4.3 cases per month and 16 cases were tested in August and September 2012 with an average of 8 cases per month. MMR protein loss was identified in 6 of $26(23 \%)$ cases in group 1 and in 3 of $16(19 \%)$ cases in group 2 . The average age of patients with MMR loss was 53 years compared to 55 years for patient with preserved MMR proteins $(\mathrm{p}=0.5493)$. One patient in-group 2 was 64 years old. Eight of $9(89 \%)$ tumors with MMR protein loss showed endometrioid morphology and one showed clear cell morphology. Of the 33 tumors with preserved MMR protein, 29 (88\%) showed endometrioid morphology, and 1 each was serous, carcinosarcoma, and mixed endometrioid and clear cell carcinoma. Eight of $9(88 \%)$ tumors with MMR loss were FIGO stage 1, and one was FIGO stage 2 . The tumors with preserved MMR protein were also predominantly stage $1(26 / 33,79 \%)$ No statistical difference was identified with respect to stage $(\mathrm{p}=0.6622)$

Conclusions: Universal MMR protein testing doubles the workload of pathology laboratory, but does identify patients with possible Lynch syndrome that are not normally suspected based on patient's age.

1984 Biopsies

Results of a Quality Assurance Program for Needle Core Breast

SJ Kantola, NS Campbell, GL Keeney, DW Visscher, RG Gamez. Mayo Clinic, Rochester, MN.

Background: The diagnostic accuracy of needle core breast biopsy is unknown and methods for determining accuracy and quality assurance (QA) have not been well developed.

Design: Daily review of breast core biopsies was performed within 24 hours of sign-out, by a breast pathologist who was blinded to the initial diagnosis. The study period was 45 months. Disagreements were classified as major (MA) or minor (MI) depending on potential for clinical impact as discussed with treating physicians. All major 
disagreements were arbitrated by a third pathologist. Pathology reports were amended as necessary. Chart review was done continuously to determine clinical follow up and open excision diagnosis (DX)

Results: Of the 2538 cases reviewed, 53 (2.1\%) had a MA and 159 had a (6.3\%) MI disagreement between the original and QA review pathologist. Of the MA disagreements, 14 were considered to be "significant" based on their potential effect on management. Table 1: Major Disagreements

\begin{tabular}{||l||l|l||l|l||}
\hline \hline & $\mathbf{N}(\%)$ & $\begin{array}{l}\text { Excision agrees } \\
\text { W/original (\%) }\end{array}$ & $\begin{array}{l}\text { Excision agrees } \\
\text { W/QA (\%) }\end{array}$ & Undetermined (\%) \\
\hline Atyp to Benign & $7(13.2)$ & $3(42)$ & $2(29)$ \\
\hline Benign to Atyp & $22(41.5)$ & $3(13)$ & $8(37)$ & $2(29)$ \\
\hline Atyp to Atyp & $1(1.8)$ & 0 & 0 & $11(50)$ \\
\hline Benign/Atyp to DCIS & $3(5.7)$ & 0 & $2(67)$ \\
\hline DCIS to Benign/Atyp & $2(3.8)$ & 0 & $1(50)$ & $1(100)$ \\
\hline FA to FEL & $11(20.8)$ & $4(37)$ & 0 & $1(33)$ \\
\hline FEL to FA & $4(7.6)$ & $2(50)$ & $2(50)$ & $7(63)$ \\
\hline Incvasive to Benign/Atyp & $1(1.8)$ & 0 & $1(100)$ & 0 \\
\hline Other & $2(3.8)$ & & & 0 \\
\hline Invasive ductal carcinoma & & $1(100)$ & 0 & 0 \\
\hline to DCIS & & 0 & 0 & $1(100)$ \\
\hline $\begin{array}{l}\text { Benign to Suspicious for } \\
\text { cancer }\end{array}$ & & 0 & \\
\hline
\end{tabular}

Atyp: Atypical, DCIS: ductal carcinoma in situ. FA: Fibroadenoma, FEL: Fibroepithelial lesion Original DX was done by a general pathologist in $42 \%$, general + breast in $26 \%$, breast only in $17 \%$ and 2 breast pathologists in $16 \%$ of the cases. Incidence of MA disagreements was higher when a breast specialist was involved in the original DX $(58 \%$ vs. $42 \%$ ). The excision DX agreed with the original in $24.5 \%$, with the QA reviewer in $30.5 \%$ and was undetermined in $45 \%$ of the cases. Undetermined cases were due to loss to follow up $(66 \%)$, lack of excision DX $(17 \%)$ or lack of gross-radiology correlation on excision $(17 \%)$. Lack of clinical significance for many "major" disagreements reflects complex clinical scenarios in which multiple biopsies were performed in patients with known cancer.

Conclusions: 1) Major disagreements centered on intrinsically problematic lesions such as focal atypia, low grade DCIS and cellular fibroepithelial tumors. 2) The data demonstrate that a secondary review diagnoses should not always be viewed as "correct". 3) Determining "correct" diagnosis in problematic cases is often difficult owing to small lesion size, complex clinical scenarios or incomplete clinical follow up.

1985 Does Expertise in Breast Pathology Influence the IntraOperative Diagnosis of Breast Sentinel Lymph Nodes? An Institutional Experience

A Kumarapeli, B Song, L Balos, E Marchetti. State University of New York at Buffalo, Buffalo, NY.

Background: Metastatic carcinoma in a sentinel lymph node(s) (SLN) dictates the operative strategy for breast surgeons. In institutions still performing intra-operative (IO) SLN evaluation, a positive SLN will lead to an axillary dissection for the patient at the time of initial surgery. SLN evaluation in this setting is influenced by many factors, including sampling, tissue variability, and interpretation intraobserver variation. We sought to analyze the discordance rates on IO SLN diagnoses made by a general pathologist versus a breast pathologist.

Design: A computer based retrospective search identified 140 breast carcinoma case with SLNs that were signed out at the Buffalo General Medical Center during a two year period, July 2010-June 2012. 129 cases had SLNs sent for IO assessment. We analyzed the discordance rate of the $\mathrm{IO}$ and permanent diagnoses as a quality assurance measure. Results: A total of 140 SLNs were examined; 35 were positive $(25 \%)$ for metastatic carcinoma. 129 of 140 SLNs were analyzed intra-operatively; 29 SLNs showed metastatic carcinoma on permanent sections. Of these 29, 14 were identified during IO evaluation. There were 15 false negative IO diagnoses of SLNs, a $52 \%$ false negative rate; with 3 macrometastases, 5 micrometastases and 7 isolated tumor cells (ITC) later identified on permanent sections. In this study, the sensitivity and specificity of intra-operative analysis is $48 \%$ and $100 \%$, respectively. In this institution, IO evaluation demonstrated 114 of 129 concordant SLN diagnoses overall. When data was separately analyzed for breast pathologists versus general pathologists, breast pathologists performing IO diagnoses had a discordant rate of $7.5 \%$ compared to the general pathologist which was $16.1 \%$.

Conclusions: We found an overall SLN diagnosis discordance rate of $11.6 \%$, but only $6.2 \%$ when ITCs were excluded. In our review, breast pathologists have a lower discordant rate in the IO assessment of SLNs, when compared to the general pathologist. Although, this study is limited by a small sample size, we conclude that expertise in breast pathology will enhance the intra-operative diagnostic accuracy of SLNs.

\section{CD123 Is a Useful Immunohistochemical Marker for Colonic} Graft Versus Host Disease

J Lin, S Chen, Z Zhao, OW Cummings, R Fan. Indiana University School of Medicine, Indianapolis, IN.

Background: Hematopoietic stem cell transplantation provides a potential cure for many immunohematopoietic disorders. However, the efficacy of this treatment is greatly hampered by graft versus host disease (GVHD) and opportunistic infection. The gastrointestinal tract is one of the main target organs for GVHD and infections, particularly in form of cytomegalovirus colitis. Cytomegalovirus infections can coexist or superimpose GVHD, sometimes during anti-GVHD treatment. The presence of crypt apoptosis is one of the major criteria when diagnosing GVHD; however, it is also common finding in cytomegalovirus colitis. Thus, the definitive histopathological diagnosis of gastrointestinal GVHD is often challenging or impossible without reliable ancillary markers. Some previous studies suggested a role of CD123 and C4d in intestinal GVHD pathophysiology.
Design: In this study, we retrospectively reviewed 38 colonic biopsies from 34 patients who received stem cell transplantation and had a diagnosis of GVHD ( 20 cases of grade 1-2 and 18 cases of grade 3-4) and 14 colon biopsies from 14 patients who had cytomegalovirus colitis without history of stem cell transplantation, and studied the expression of CD123 and C4d in both groups.

Results: Our data demonstrated significantly increased expression of CD123 in the GVHD group (total $65.8 \% ; 60 \%$ in grade $1-2 ; 72.2 \%$ in grade $3-4$ ) compared to the cytomegalovirus group $(14.3 \% ; \mathrm{p}<0.01)$; there was no significant difference of $\mathrm{C} 4 \mathrm{~d}$ expression between the two groups ( $68.4 \%$ and $42.9 \%$ respectively; $\mathrm{P}>0.05)$.

Conclusions: Our study indicates that for patients who are highly suspicious for gastrointestinal GVHD in the presence of confounding factors, CD123 might be a useful ancillary marker.

\section{Childhood Esophagitis - A Practice Audit}

MC Lynch, KA Millington, H Mani. Penn State Hershey Medical Center, Hershey, PA. Background: Consensus recommendations for diagnosis and treatment of eosinophilic esophagitis were published in 2007 . We performed a clinicopathologic analysis and practice audit of childhood esophagitis to evaluate implementation of recommendations in a tertiary care academic center.

Design: All esophageal biopsies performed in children in a one-year period were reviewed and cases with esophagitis selected for study. Pathology slides were semiquantitatively graded for esophagitis as recommended (based on basal cell hyperplasia, papillary elongation and spongiosis) and evaluated for recommended elements (inflammation, eosinophil numbers and fibrosis). Medical charts were reviewed for all available clinical and laboratory information.

Results: Of 780 esophageal biopsies performed in the one-year-period, $208(26.7 \%)$ had esophagitis. Mean patient age was $9.18 \mathrm{y}$ (range 11d to $18 \mathrm{y}$ ), with a male dominance. Epithelial eosinophilia and submucosal fibrosis correlated with increasing esophagitis grades. All grades showed mild lymphocytosis. Submucosal inflammation, when present, was predominantly lymphocytic, with eosinophil dominance in $8 / 108(7.4 \%)$ cases. Lymphoid aggregates were seen in all grades. These pathologic parameters were rarely included in the original pathology reports.

Pathologic Features

\begin{tabular}{|c|c|c|c|c|c|}
\hline Grade (n) & Eosinophils/hpf & $\begin{array}{l}\text { Eosinophil } \\
\text { Microabscess }\end{array}$ & $\begin{array}{l}\text { Lymphocytes/ } \\
\text { hpf }\end{array}$ & $\begin{array}{l}\text { Lymphoid } \\
\text { Aggregates }\end{array}$ & $\begin{array}{l}\text { Submucosal } \\
\text { Fibrosis }\end{array}$ \\
\hline Minimal (32) & 3.7 & $0 / 32$ & 9 & $4 / 8$ & $1 / 8$ \\
\hline Mild (75) & 13 & $8 / 75$ & 17 & $8 / 31$ & $9 / 31$ \\
\hline Moderate (64) & 39 & $33 / 64$ & 18 & $14 / 34$ & $24 / 34$ \\
\hline Severe (37) & 88 & $34 / 37$ & 18 & $6 / 25$ & $17 / 25$ \\
\hline
\end{tabular}

Clinical details were available in $172(82 \%)$ cases. 103/172 (60\%) cases had esophageal dysfunction (pain or dysphagia). Dysphagia was comparable between grades, while pain decreased with increasing esophagitis. Heartburn was more frequent in minimal esophagitis, probably reflecting reflux esophagitis. Endoscopic findings were available for $151(73 \%)$ cases, and was abnormal in 84/151 (56\%) cases. Blood eosinophilia was uncommon. Allergen details were not available for any case.

Clinical Features

\begin{tabular}{|c|c|c|c|c|c|c|c|}
\hline Grade (n) & $\begin{array}{l}\text { Mean } \\
\text { age }\end{array}$ & $\begin{array}{l}\text { Gender } \\
(\mathrm{M}: \mathrm{F})\end{array}$ & Pain & Dysphagia & Heartburn & \begin{tabular}{|l} 
Abnormal \\
EGD
\end{tabular} & $\begin{array}{l}\text { Blood } \\
\text { Eosinophilia }\end{array}$ \\
\hline Minimal (32) & 9.26 & $20: 12$ & $14 / 25$ & $4 / 25$ & $20 / 25$ & $11 / 20$ & $1 / 15$ \\
\hline Mild (75) & 9.16 & $47: 28$ & $33 / 60$ & $9 / 60$ & $27 / 60$ & $22 / 54$ & $2 / 32$ \\
\hline Moderate (64) & 9.81 & $45: 19$ & $20 / 53$ & $10 / 53$ & $24 / 53$ & $29 / 46$ & $1 / 27$ \\
\hline Severe (37) & 8.37 & $26: 11$ & $9 / 34$ & $4 / 34$ & $19 / 34$ & $22 / 31$ & $1 / 20$ \\
\hline
\end{tabular}

Conclusions: Consensus recommendations are not uniformly followed at either the clinical or pathology levels, thus diluting the utility of published guidelines. Wider awareness of guidelines and synoptic reporting will allow uniform assessment of pediatric esophageal biopsies and may help close gaps in disease knowledge.

\section{Comparison of the ThinPrep ${ }^{\circledR}$ Imaging System Versus Total} Manual Rescreening

EL Martin, CW Michael, S Shyu, P Bomeisl, J Wasman. University Hospitals Case Medical Center, Cleveland, OH.

Background: The ThinPrep ${ }^{\circledR}$ Imaging System (TIS) is FDA approved for screening Papaniculou (Pap) tests using 22 fields of view (FOV) considered most abnormal by the TIS. Some controversy exists in the cytopathology community whether screening using the TIS is adequate in cases diagnosed as negative (NIL) or whether manual rescreening should be performed on all cases. Therefore, we compared the performance of screening with the TIS to total manual rescreening in NIL Pap tests.

Design: 4341 ThinPrep ${ }^{\circledR}$ Pap tests were screened using the TIS. 3340 were diagnosed as NIL on the 22 FOV selected by the TIS and subsequently manually rescreened by the same cytotechnologist. 674 cases were sent to a cytopathologist for review and final diagnosis. Biopsy follow up and Human Papilloma Virus (HPV) test results were noted if available for any Pap test with a final diagnosis of atypical squamous cells of undetermined significance (ASCUS) or above.

Results: Of the 3340 diagnosed as NIL on the TIS, 3159 (94.6\%) were confirmed NIL on manual rescreen. This includes 493 of the 674 (73.1\%) cytopathologist reviewed Pap tests. The false-negative rate of screening with the TIS was $5.4 \%$. Of the 181 Pap tests that were signed out as abnormal, 147 were ASCUS, 6 were atypical squamous cells - cannot exclude high grade (ASC-H), 9 were atypical glandular cells (AGUS), 19 were low grade (LSIL), and 0 were high grade (HSIL). Biopsy follow up and HPV results are presented in table 1 . Of the 148 with HPV results, $43(29 \%)$ were positive. Of the ASCUS and HPV+ cases, only 1 cervical intraepithelial neoplasia (CIN) 2 was found on biopsy follow up. 


\begin{tabular}{|c|c|c|c|c|}
\hline Final Diagnosis & {$\left[\begin{array}{l}\% \text { of Abnormals } \\
(\mathrm{n}=181)\end{array}\right.$} & $\begin{array}{l}\% \text { of NIL on TIS } \\
(\mathrm{n}=3340)\end{array}$ & Biopsy Follow Up & Positive for HPV \\
\hline ASCUS & $81.2(147)$ & 4.4 & $7 / 11$ CIN1, $1 / 11$ CIN2 & $39 / 132$ \\
\hline ASC-H & $3.3(6)$ & 0.18 & $1 / 6$ CIN1 & $1 / 6$ \\
\hline AGUS & $5.0(9)$ & 0.27 & $0 / 3 \mathrm{CIN}$ & $0 / 5$ \\
\hline LSIL & $10.5(19)$ & 0.57 & $7 / 10 \mathrm{CIN} 1$ & $3 / 5$ \\
\hline
\end{tabular}

Conclusions: Based on our data, the TIS for screening of Pap tests is accurate and reliable in NIL cases as compared to total manual rescreening. Most of the false-negative cases $(81.2 \%)$ were diagnosed as ASCUS on subsequent review, with 0 HSIL cases. On patients with available follow-up, only 1 case was diagnosed as greater than CIN1 on biopsy. The HPV+ rate of ASCUS cases missed on screening with the TIS was $29.5 \%$, suggesting that ASCUS may have been over diagnosed in some of these cases. Our results confirm that the TIS is highly accurate in excluding HSIL, negating the need for total manual rescreening.

1989 Photography Is an Underutilized Resource Crucial to Documentation of Cause of Death and Assurance of High Quality Postmortem Examinations

B Mathis. Miami-Dade County Medical Examiner Department, Miami, FL.

Background: Most consider autopsy as the gold standard for both the determination of cause of death and the definitive documentation of antemortem pathology and injury. At the time of autopsy, pathologists have but one opportunity to properly document and preserve the findings. Our institution, a high-volume metropolitan medical examiner office, has a dedicated forensic photographer available throughout all cases. This service is often not available at smaller offices and sites. The purpose of this study is to quantify the proportion of autopsy findings that are photographed from cases with a natural manner of death.

Design: We performed a retrospective review of autopsy records from 104 decedents with a natural-manner death in 2011. The "findings" section of each autopsy report was compared with its associated photographs to determine how many of the findings had been photographed, and whether an anatomic manifestation of the ultimate cause of death had been captured. Additionally, we surveyed how frequently common pathologic findings such as cardiomegaly, coronary artery disease, and pulmonary edema, amongst others, were photographed.

Results: The number of findings in the "findings" section ranged from 2 to 17 ( mean=7.3, median=7), with the number photographed ranging from 0 to 10 (mean=2.6, median $=2)$. On average $34 \%$ of the autopsy findings were photographed from each case (median $=29 \%$ ). Additionally, anatomic manifestations of the cause of death were photographed in $59 \%$ of cases $(n=61)$. For example, among decedents with hypertensive heart disease certified as a cause of death $(\mathrm{n}=36), 81 \%(\mathrm{n}=29)$ had cardiomegaly and $89 \%$ $(\mathrm{n}=32)$ had nephrosclerosis. $42 \%(\mathrm{n}=12)$ of such cases had photographic documentation of the heart, while $28 \%(n=9)$ had of the kidneys.

Conclusions: Our study shows that even at an office with a dedicated photographer in the morgue, natural-manner death cases often do not have accompanying photography. For each case, approximately one-third of the findings were photographed, and 59\% of these photographs demonstrated evidence relating to the cause of death. Gross photography is perhaps the most efficient and objective means of preserving the findings observed during an autopsy. New information may come to light in the future, or questions regarding the interpretation of the findings may arise at a later time, necessitating review of gross findings. Therefore, gross photography plays a key role in objectively immortalizing autopsy findings to insure the quality and reproducibility of the postmortem examination.

\section{Effectiveness of Pathology Resident Communication} Simulation Based Education on Clincial Practice

S Mehri, A Wingerson, J Struijk, D Luff, S Raab, S Dintzis. University of Washington Medical Center, Seattle, WA.

Background: In the frozen section room, effective delivery of a diagnosis has immediate impact on patient care. In attempt to train residents in communication skills, we developed communication training modules using simulated clinician-pathologists interactions and checklists with 14 communication elements for performance evaluation. The modules are based on TeamSTEPPS, a nationally implemented evidence-based strategy used for improving communication and teamwork skills among health care professionals. In order to evaluate the current intraoperative consultation practice at our institution and establish a baseline for our training studies, intraoperative communications were audio recorded to determine if basic communication elements on our evaluation checklist were being performed.

Design: Thirty-six intraoperative consultations were recorded throughout a 2 month period and retrospectively reviewed. The conversations involved 15 pathology attendings and 21 pathology residents. Of the 21 resident conversations recorded 8 involved residents who had undergone our TeamSTEPPS-based communication training. The conversations were scored according to whether standard communication elements were performed, including patient, self and clinician identification; read-back confirmation, and clinician needs assessment. Additionally, we compared resident performance during actual intraoperative consultations with the pre- and post-training performance of residents who participated in our simulation-based communication training.

Results: Attending pathologists achieved an average score of $80 \%$ on completion of checklist items during recorded intraoperative consultations. Residents who had undergone communication training scored an average of $84 \%$. Non-trained residents scored an average of $66 \%$. Though trained residents outperformed attendings and untrained residents on all checklist items, their overall performance during actual intraoperative consultations was lower than during their simulation-based assessment post-training ( $84 \%$ and $94 \%$, resp.) and higher than their initial baseline assessment pre-training $(77 \%)$.

Conclusions: Effective delivery of pathology results can be as important as an accurate diagnosis in the intraoperative setting. Miscommunications may be reduced by ensuring that basic introduction, content and immediate acknowledgement is routinely performed. Simulated resident-clinician communication training modules improve resident communication skills as measured by both post-training simulation testing and evaluation of actual intraoperative consultations.

1991 Diagnosing Ambiguous Melanocytic Tumors in a Tertiary Referral Center: The Contribution of Fluorescence In Situ Hybridization (FISH) to Conventional Histopathologic and Immunophenotypic Analyses TL Milless, CL Jonathan, T-CA Carlos, W Wei-Lien, MS Michael, I Doina, PG Victor TT Michael. UT MD Anderson Cancer Center, Houston, TX.

Background: The distinction of benign from malignant melanocytic proliferations remains a diagnostic challenge with critical clinical implications. The current diagnostic algorithm includes assessment of histopathologic (architecture, cytology, mitotic activity) and immunohistochemical (HMB-45 and Ki-67) parameters. Recently, FISH has been reported as a useful ancillary diagnostic tool in melanocytic lesions, but most studies have assessed the performance of FISH in unequivocal melanomas and nevi. Thus, the diagnostic utility of FISH in ambiguous melanocytic tumors remains controversial.

Design: 32 histopathologically ambiguous melanocytic proliferations from our consultation service were reviewed. The histopathologic, immunophenotypic and FISH parameters (NeoGenomics; using probes for 6p25, 6q23, 11q13 and Cen6) informing our diagnostic impression were correlated with the final consensus diagnosis and clinical follow-up.

Results: 32 melanocytic tumors from 13 females and 18 males with a mean age of 28 years (range: 2 to 64) were examined. Prior to FISH testing, cases were designated as "favor benign" $(\mathrm{n}=22)$ or "favor malignant" in $(\mathrm{n}=10)$ by a consensus group (up to 7 dermatopathologists) based on an assessment of clinical, histopathologic and immunophenotypic parameters. FISH testing was positive in $3 / 22$ "favor benign" lesions (two with a pattern consistent with polypoloidy) and in $5 / 10$ "favor malignant" lesions. In all 32 cases, the final diagnosis correlated with the initial consensus impression rendered independently of FISH. In this series of melanocytic tumors, the sensitivity of FISH for the diagnosis of melanoma was $50 \%$; the specificity was $86.4 \%$; the positive predictive value (PPV) was $62.5 \%$; the negative predictive value (NPV) was $79.2 \%$. Follow-up (range: $2-21$ months) was available for 25 patients (17/8 with benign/malignant diagnosis, respectively). Among benign lesions: 2 were not treated, 15 underwent wide local excision (WLE), and a single patient also underwent sentinel lymph node sampling (SLNS) which was negative for metastasis. Among the 8 malignant lesions, all underwent WLE-SLNS. A single lymph node metastasis was identified in a 4 year old girl with FISH-negative melanoma.

Conclusions: The diagnosis of ambiguous melanocytic tumors requires a multi-faceted assessment of clinical, histopathologic and immunophenotypic parameters. Given its apparent low sensitivity and PPV in the setting of ambiguous lesions, FISH testing does not significantly impact the final diagnosis.

$1992 \quad$ Evaluating Peripheral Blood Lymphocytosis: Improving Efficiency While Maintaining Quality

AS Morgan, $V$ Tseng, CP Leith, DT Yang. University of Wisconsin, Madison, WI; Emory University, Atlanta, GA.

Background: Peripheral smear review is a critical adjunct to automated CBC analysis when abnormalities are identified. Smear review is a large contributor to overall laboratory cost and productivity. An absolute lymphocyte count (ALC) $>5000 / \mathrm{uL}$ is the consensus guideline for review of lymphocytosis. We hypothesize that evidence based adjustment of ALC and age discriminators will decrease the workload without compromising patient care.

Design: $1100 \mathrm{CBCs}$ with ALCs $>5000 / \mathrm{uL}$ analyzed in the core hematology laboratory for a two year period (2008-9) were included. Health records were reviewed and patients were categorized into diagnostic groups based on strictly defined follow-up criteria. 401 patients with new onset lymphocytosis who met criteria for reactive lymphocytosis $(82 \%)$ or lymphoproliferative disorder $(18 \%)$ were identified. Receiver operating characteristic (ROC) curve analysis was used to establish optimal ALC and age cut-offs for this group.

Results: As an initial screening discriminator between benign and disease populations, ALC has an ROC area under the curve (AUC) of 0.725 indicating a moderate degree of overlap between these two groups. Selecting cases with ALC $>10,000 / \mathrm{uL}$ for review enriched the proportion of lymphoproliferative cases in the review pool (specificity $=$ $90 \%$ ) but decreased sensitivity (sensitivity $=52 \%$ ). For this group of cases with ALC between 5,000 and $10,000 / \mathrm{uL}$, age could be utilized as a disease discriminator, with an ROC AUC of 0.886 . 

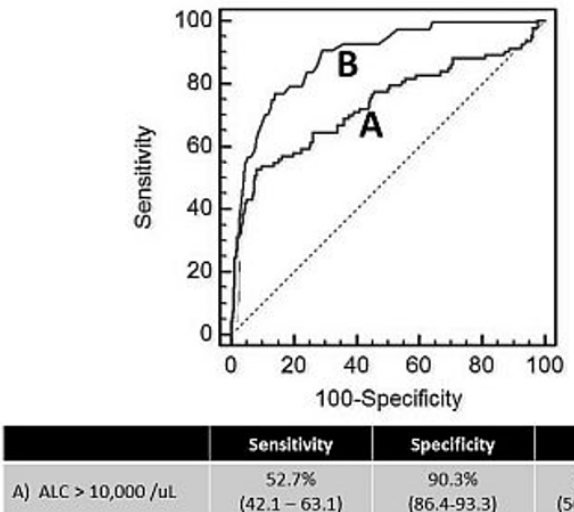

PPV

NPV

B) Age $>50$ years $(42.1-63.1)$ (86.4-93.3) $93.2 \%$ $62.0 \%$ $28.1-89.9100$ [81.3-98.6)

$27.9 \%$ $98.3 \%$ (95.1-99.6)

Selecting patients $>50$ years old in this group for review efficiently captured the disease population with a sensitivity and specificity of $93 \%$ and $62 \%$, respectively. Implementing these review criteria would have excluded $60 / 401$ cases $(15 \%)$ from review, including three lymphoproliferative cases, none of which have required treatment to date. Conclusions: To our knowledge, this is the first retrospective cohort study evaluating all patients with newly discovered lymphocytosis, not just patients who underwent flow cytometry, for predictors of lymphoproliferative disorders. Our findings suggest that modifying the typical 5000/uL ALC smear review threshold through retrospective analysis of institutional data can reduce the laboratory workload without compromising quality.

1993 Root Cause Analysis of Discordant and Deferred Frozen Section Diagnoses

SA Mortuza, M Bonert, B Rowe, FX Torres, SS Raab. Eastern Health and Memorial University of Newfoundland, St. John's, Newfoundland, Canada.

Background: Frozen section (FS) intraoperative consultation provides information that appropriately guides patient care. We examined failures in pathology steps leading to discordant and deferred FS diagnoses using a hierarchical root cause method that categorized cause into active and system components related to the pathology process steps. From these data, we derived a rule book/checklist for improved practice.

Design: We performed a retrospective review of 427 consecutive FS cases from the past 3 years to retrieve $11(2.5 \%)$ discordant and $15(3.5 \%)$ deferred cases. These failures first were classified by originating process step: gross sampling, technical, interpretation, and reporting. We then used an Ishikawa fishbone diagram to classify root causes into the 6 categories of human factor, methods, equipment, materials, measures, and culture. We then examined the groups of causes to determine interventions specifically focusing on lowering system noise resulting in ambiguous signals for pathologist interpretation and introducing cognitive task rules that would assist pathologists in producing the highest quality diagnosis.

Results: We classified the failure origination step as 8 in the gross sampling and technical, 13 in the interpretation, and 5 across all. Seven failures had tumor on permanent sections (no tumor on FS), but our evaluation showed contributing factors originating in the sampling phase. The fishbone diagram showed large numbers of contributing causes for all errors in the categories of human, methods, measures, and culture components. Contributing human factor causes included insufficient decision making education (22), cognitive bias (4), and insufficient technical skill education (3). All failures had cultural causes, including 9 cases in which the pathologist lacked appropriate back-up expertise. All failures had multiple method component causes, as no existing practice rules or procedures were in place. The developed checklist included rules for all process steps (e.g., sampling rules for specific cases) and incorporated system changes affecting the 6 categories of root cause.

Conclusions: All failures in intraoperative FS had a large number of contributing causes generally related to system processes. Human factor components had a large role in most failures and generally related to failures in decision making based on communication with limited data, bias, and lack of system support. We hypothesize that our rule book/checklist will lower specific failure types by targeting root causes that are systemic rather than individual.

1994 Institutional Consultations for Continuation of Patient Care: An Audit of 4 Years Including $\mathbf{3 1 9 3}$ Cases from a Single Institution

$K G$ Neill, S Zee, S Hwang, M Singh, C Tornos. Stony Brook University Hospital, Stony Brook, NY.

Background: Mandatory review of outside diagnostic surgical pathology material is standard institutional policy in the majority of medical centers before starting any kind of therapy. The Association of Directors of Anatomic and Surgical Pathology (ADASP) issued guidelines for "consultations in surgical pathology," referring to these as "institutional consultations" to distinguish them from "personal consultations". ADASP guidelines state that newly issued reports on these cases should not only be sent to the clinicians in the second institution, but to the original reporting pathologist as well. If significant disagreement occurs, the case should be discussed with the original pathologist.
Design: The aim of this study was to assess the rate of major diagnostic discrepancies for cases sent from our institution to other medical centers, compliance regarding notification of the original pathologist about diagnostic discrepancies, and compliance with sending copies of pathology reports to the original institution in a 4 year period. This information was obtained from records kept in our institution when slides are sent to other centers, and our QA logs. A "major discrepancy" was defined as any change in diagnosis implying a different treatment or prognosis.

Results: From January 1, 2008 to December 12, 2011, our department issued reports on 83,320 surgical pathology cases. $3193(3.8 \%)$ cases were sent to other medical centers for continuation of care. Of these, $2827(88.5 \%)$ were sent to large hospitals/academic centers, and $366(11.5 \%)$ were sent to community hospitals. Reports were received from institutions in only 1644 cases $(51.4 \%)$, including 1458 from large centers $(51.5 \%)$ and 186 from community hospitals $(50.8 \%)$. There were two cases with major diagnostic discrepancies $(0.06 \%)$, although no harm came to the patients. Of these two, in only one case did the reviewing pathologist notify and discuss the discrepancy over the phone with the contributing pathologist.

Conclusions: When reviewing institutional consult cases, hospitals are not compliant with sending copies of reports to the contributing pathologist in more than $49 \%$ of cases. Pathologists reviewing institutional consultations are not compliant with notifying the contributing pathologist of major diagnostic discrepancies. Because follow up reports were not received for almost half of the cases, the number of cases with diagnostic discrepancies is unkown. An effort has to be made by directors of surgical pathology laboratories to emphasize the importance of such communications.

1995 Feasibility of Levey-Jennings Quality Control Charts for Monitoring Estrogen Receptor (ER) and Progesterone Receptor (PR) Immunohistochemistry Testing

H Neufeld, R Berendt, D Pilavdzic, B Ziola, J Krahn, C Cheung, B Gilks, J Garratt, $S$ Carlsen, E Torlakovic. University of Saskatchewan, Saskatoon, SK, Canada; Cross Cancer Institute/University of Alberta, Edmonton, AB, Canada; Jewish General Hospital, McGill University, Montreal, QC, Canada; University Health Network, University of Toronto, Toronto, ON, Canada; Vancouver General Hospital, University of British Columbia, Vanocuver, BC, Canada; Lions Gate Hospital, Vancouver, BC, Canada. Background: Clinical immunohistochemistry (IHC) techniques are not yet fully standardized. Quality Control (QC) chart analysis has traditionally been applied to liquid-based laboratory testing but not to clinical immunohistochemistry (IHC) laboratories. Image analysis (IA) introduced a true quantitative aspect to interpretation of IHC results, which now makes possible, the theoretical application of control charts to clinical immunohistochemistry quality control. In this project, we tested the real world feasibility of applying QC charts in monitoring the daily performance of positive controls in clinical IHC.

Design: IA was used to evaluate both usual human tissue-based and cell line-based positive controls for estrogen receptor (ER) and progesterone receptor (PR). Several series of on-slide tissue and cell-line positive controls were cut onto the same slide, stained for ER and/or PR, digitally scanned (Aperio ScanScope), and then assessed using IA algorithms. The readings of sequential control samples were plotted as LeveyJennings QC charts.

Results: Levey-Jennings charts consisting of IA-generated H-scores were created for both tissue-based and cell line-based positive controls. Cell line-based positive controls were a better reflection of true protocol variations since they do not incorporate error due to tissue heterogeneity that is an intrinsic component of human tissue-based positive controls.

Conclusions: Levey-Jennings QC charts are feasible for clinical IHC. They are an excellent way to observe trueness, reproducibility, and repeatability of the IHC protocols.

1996 Ranking of Individual Laboratory Performance in Immunohistochemistry Proficiency Testing Using Digital Image Analysis H Neufeld, D Pilavdzic, J Garratt, B Gilks, C Cheung, B Ziola, R Berendt, J Krahn, S Carlsen, E Torlakovic. University of Saskatchewan, Saskatoon, SK, Canada; Jewish General Hospital, McGill University, Montreal, QC, Canada; Lions Gate Hospital, Vancouver, BC, Canada; Vancouver General Hospital, University of British Columbia, Vancouver, BC, Canada; University Health Network, University of Toronto, Toronto, ON, Canada; Cross Cancer Institute/University of Alberta, Edmonton, AB, Canada. Background: Various methods can be used for monitoring laboratory performance in proficiency testing (PT) in immunohistochemistry. Currently, Canadian Immunohistochemistry Quality Control (CIQC), PT results are evaluated by a panel of "expert assessors" including pathologists and technologists. The results of the assessments are used to calculate the sensitivity, specificity and kappa values in order to evaluate accuracy of IHC tests. In this study, we compared i) the rankings of participating laboratories using digital image analysis (IA)-based scoring vs. those derived by usual expert assessment, and ii) calibrated and standardized cell line microarrays (CLMAs) vs. human tissue-based TMAs.

Design: Both assessment methods evaluated two consecutive Canada-wide PT runs for estrogen receptor (ER), progesterone receptor (PR) and HER2. Newly calibrated and standardized cell line microarrays (CLMAs) were built from formalin-fixed paraffin-embedded cell pellets from nine human breast cancer cell lines. Laboratory Score Reference Score Ratios (LSRSR) were calculated using H-scores generated from both cell line- and tissue-based samples. Participating laboratories LSRSRs were compared to the results obtained by 6 reference laboratories, which range was used as acceptable standard. 
Results: Laboratory ranking by IA was not significantly different than by ranking by expert assessment. CLMAs were better discriminators than tissue-based samples. Cell lines with intermediate $\mathrm{H}$-scores were more sensitive in detecting interlaboratory variation than those with either very high or very low $\mathrm{H}$-scores. The IA-based method also allowed for the monitoring of performance trends for each participating laboratory over multiple successive runs.

Conclusions: IA-based evaluation of PT provides useful information regarding monitoring of protocol calibration. This assessment method requires only a small number of cell line-based samples. This approach also significantly reduces the time and costs of PT evaluation.

\section{Application of Image Analysis for Daily Monitoring of Positive} Controls in Clinical Immunohistochemistry

H Neufeld, R Berendt, D Pilavdzic, B Perez-Ordonez, B Ziola, B Gilks, J Garratt, C Cheung, J Krahn, S Carlsen, E Torlakovic. University of Saskatchewan, Saskatoon, SK, Canada; University of Alberta, Edmonton, AB, Canada; McGill University, Montreal, QC, Canada; University of British Columbia, Vancouver, ON, Canada; Lions General Hospital, Vancouver, BC, Canada; University of Toronto, Toronto, ON, Canada.

Background: Digital Image Analysis (IA) is a relatively new application for the evaluation and quantification of clinical immunohistochemistry (IHC) tests. Calibrated standardized positive controls are especially desirable for Class II IHC markers that are quantitative in nature. This study compares the use of image analysis by evaluating i) the effect of tissue surface area on reproducibility, and ii) the use of cell line-based vs. tissue-based positive controls in clinical IHC practice for estrogen receptor (ER) and progesterone receptor (PR)

Design: The Aperio Scanscope IA system was used to evaluate tissue-based controls made from human tissues. Formalin-fixed paraffin embedded cell line-based controls from selected human breast cancer cell lines were also stained and compared. Groups of sequential, currently used, on-slide IHC controls of human tissue-based controls from four major Canadian academic laboratories were examined to observe the effects of tissue types (breast, endometrium, cervix) and surface area of tissue samples. IA results of whole tissue sections were compared with results derived from only a portion of tissue sections.

Results: A statistically significant variation of $\mathrm{H}$-scores was observed between independent IHC runs using all tissue-based controls for both ER and PR (F-test, $\mathrm{p}<0.01$ and F-test, $\mathrm{p}<0.01$ respectively). Comparison with cell line-based control samples showed that the variation was largely due to tissue heterogeneity, with little to no contribution by tissue type. However, tissue size was an important determinant of variation with larger tissue samples exhibiting less variation than smaller tissue samples. Conclusions: IA can be used for daily monitoring of IHC QC. However, the selection of control type and tissue surface area are important factors in determining control performance. Cell line-based controls were superior to human tissue-based controls due to their greater homogeneity. However, if tissue controls are monitored by IA, then IA evaluation should be performed on larger (rather than smaller) tissue sections in order to reduce the effects of tissue heterogeneity on results.

\section{Quantitative Evaluation of ER alpha Expression in Breast Cancer Using DAB Based Immunohistochemistry Versus Quantitative Immunofluorescence}

VM Neumeister, E Zarrella, M Coulter, K Schalper, D Carvajal, DG Hicks, DL Rimm. Yale University School of Medicine, New Haven, CT; University of Rochester, School of Medicine, Rochester, NY.

Background: An accurate, sensitive, reproducible and reader independent method to measure ER expression is important for predicting outcome and guiding treatment options for patients. Here we compare quantification of DAB based immunohistochemistry with quantitative immunofluorescence (QIF) for assessment of ER alpha.

Design: Three different breast cancer cohorts consisting of 640, 93, and 235 patients, represented on tissue micro arrays (TMAs) were used for comparison of the two methods of ER quantification. Serial sections were stained for ER alpha. Staining procedures were optimized, standardized and automated. DAB/IHC staining was objectively quantified using the Aperio Image Analysis system. Tumor areas were annotated and 3 different algorithms were applied: the positive pixel count algorithm, the FDA cleared nuclear algorithm and a DAB concentration scoring algorithm, which assesses $\mathrm{DAB}$ concentration within a defined area. The algorithms were optimized according to Hematoxylin staining. Two pathologists performed semiquantitative scoring on the cohort of 235 patients. The serial sections stained with immunofluorescence were quantified using the Automated Quantitative Analysis (AQUA) method. Pixel intensity of ER expression is measured within the tumor area, defined by cytokeratin positivity. AQUA scores are calculated as ER intensity per tumor area.

Results: On the 3 cohorts the quantification of DAB based ER staining correlates with QIF as assessed by linear regression ( $r^{2}$ values between 0.5 and 0.85 ) with positive pixel count performing best. The nuclear algorithm and the DAB concentration scoring reveal the same limited dynamic range compared to QIF. The dynamic range (defined as the largest possible signal divided by lowest possible signal) is about 3 times larger for QIF than for DAB quantification. Based on the intensity of Hematoxylin staining several cases are misclassified and scored as false positive. In contrast, some cases showing low ER expression by QIF are negative by DAB methods, suggesting false negative results. Conclusions: QIF of ER alpha outperforms quantification of DAB based IHC when using pathologist based scores as the criterion standard. QIF also shows broader dynamic range. With increasing demands for personalized medicine and tailoring therapy according to changes of biomarker expression, accuracy, objectivity, reproducibility and dynamic range are important considerations in assay selection.
1999 Focused Professional Peer Review for Anatomic Pathology Faculty: A Pilot Study

JM Newell, RJ Zaino. Penn State Hershey, Hershey, PA.

Background: Focused and ongoing professional practice evaluations are mandated by the JCAHO and most medical staff bylaws for new and current faculty. Formal recommendations for the method of implementation do not exist in anatomic pathology, and institutional approaches vary. For gynecologic pathology, our practice was to have a member of the section review the first 200 cases prior to sign-out (concurrent review) by a new faculty member. Analysis of the most recent concurrent review showed that the ten most frequent diagnoses accounted for $73 \%$ of all cases, for which 9 of the 10 were normal or non-neoplastic. While this reflects daily practice, it provides scant information about diagnostic competency in gynecologic pathology.

Design: The goal of this project was to develop a proficiency exam for faculty members that could assess their knowledge of diagnostic gynecologic pathology. Based on the tables of contents of gynecologic pathology texts, we identified about 120 major diagnostic entities. A slide with characteristic features for each diagnosis was obtained from our archives. A representative subset of thirty cases for which the diagnosis could be made using only an H\&E stained slide were selected for the proficiency exam, of which 15 were considered routine, 13 challenging, and 2 difficult. The site, patient age, and a short clinical history were provided for each case. Current faculty in the gynecologic pathology section were given the test, for which they had 2 hours and access to desired written or electronic resources.

Results: Five faculty members with a wide range in past experience and current service frequency took the exam. Scores ranged from $21 / 30(70 \%)$ to $30 / 30(100 \%)$ with a mean of $26 / 30(87 \%)$. The scores did not appear to correlate with experience or frequency of time on service, but the sample was too small for statistical analysis. Based on existing proficiency testing for cytopathology and published discordant rates upon second review of cases, we chose a score of $90 \%$ as the threshold below which we would provide at least 22 hours of targeted CME to enhance professional development.

Conclusions: A wide range in test results was identified using this proficiency exam. This test is more efficient than concurrent review and appears to provide more useful data. With our routine case distribution, we estimate that it would require concurrent review of at least 1000 cases to achieve a similar mix of challenging and difficult diagnoses. Validation with a larger sample size using faculty at other institutions and the determination of minimal target scores and methods for remediation are future goals.

2000 A Pragmatic Approach to Assay Selection: Comparison of In-House Validated Assays Versus CE-IVD Assays for the Analysis of High-Throughput Molecular Theranostic Assays

CO'Brien, E Logan, J Gibbons, O Sheils, SE Langabeer, SP Finn. St James's Hospital, Dublin, Ireland; Trinity College, Dublin, Ireland.

Background: The recent growth and expansion of molecular pathology has encouraged a concomitant growth in the range and variety of ready-to-use CE-IVD marked assays. This increased availability of off-the shelf assays begs the question: Is it better to use in-house or CE-IVD marked assays for routine laboratory use? To find an answer to this question we designed and conducted a framework based evaluation of two comparable methods for the detection of KRAS gene mutations in colorectal carcinoma.

Design: A multifacted evaluation of two KRAS mutation detection methods - a CEIVD marked assay and an in-house assay - was conducted to determine the optimum assay for our production environment. The analysis incorporated five sequential steps 1) An unbiased literature review based on a modified ACCE system was conducted and used to identify decision criteria for assay selection.

2) Method evaluations were carried out to ensure that the assays met the decision requirements of the laboratory.

3) Failure Mode and Effects Analysis (FMEA) was conducted for each assay.

4) A cost benefit analysis (CBA) was performed for both assays.

5) Assay suitability decision in consultation with laboratory director

Results: In-house evlauation coupled with the decision criteria from the literature identified that both assays were sufficiently sensitive and had an adequate reporting range to satisfy drug adminsitration criteria. FMEA analysis showed that the in-house assay carried a greater risk of producing an incorrect result. For example, the in-house assay required approximately 435 liquid handling steps versus 58 manual liquid handling steps for the CE-IVD assay (based on a 6 sample batch size). A complete analysis of staff time and reagent costs was also undertaken which showed the reagent cost per result to be comparable between assays at the expected normal batch size. However, the in-house method required a greater staff cost.

Conclusions: By using a thorough, predifined assessment schema, we were able to conclude that the CE-IVD marked assay meets more of the requirements of our laboratory. Traditionally, method evaluations tend to focus on the analytical sensitivity of the assay or the clinical sensitivity and specificity. The above framework takes all of these factors into consideration but utilises FMEA and CBA to supplement the decision making process. This results in an assessment that accounts for all aspects of assay performance.

\section{Anaplastic Lymphoma Kinase (ALK) Copy Number Variation (CNV) as a Quality Assurance Measure for Tumor Adequacy in FISH Analysis}

NM Patel, K Kaiser-Rogers, K Rao, T Sapp, ML Gulley. University of North Carolina, Chapel Hill, NC.

Background: ALK rearrangement is found in $\sim 5 \%$ of lung adenocarcinomas. Tissue specimens used for ALK testing may contain scant tumor. Therefore, surrogate indicators of specimen adequacy are needed to ensure sufficient numbers of malignant cells are evaluated during testing. We examined whether ALK CNV, which is commonly seen in 
lung adenocarcinoma and less often in benign cells, could serve to assure that adequate tumor was present in the portion of tissue on which FISH analysis was performed.

Design: Records were obtained for all lung adenocarcinoma specimens undergoing ALK rearrangement testing using Vysis Break Apart FISH Probe at UNC Hospitals Cytogenetics Laboratory from April 2011 to August 2012. Records were reviewed for percent tumor nuclei on H\&E stained slides, percent cells with intact ALK signal CNV versus ALK rearrangement, and number of intact ALK signals per cell.

Results: A total of 184 cases were reviewed, with complete information available on 111. Two tumors had ALK rearrangement (1.1\%), and one could not be interpreted due to acid decalcification and failed hybridization. 150 cases $(81.5 \%)$ had aberrant numbers of ALK signals detected in $>20 \%$ of cells. Nine of these cases had 1 ALK allele, while 141 cases had extra ALK signals (range - 3 to 15 copies per cell). The remaining 33 cases had 2 copies of the ALK gene per cell with neoplastic cells confirmed to be present. Among cases with ALK CNV, correlation between pathologist-estimated tumor burden and proportion of cells with ALK CNV was $\mathrm{R}^{2}=0.0146$. This result suggests no linear relationship between the proportion of malignant cells as assessed on $\mathrm{H} \& \mathrm{E}$ and the proportion with ALK CNV as assessed by FISH.

Conclusions: Among tumors negative for ALK rearrangement, ALK CNV was seen in $81.5 \%$, providing support for the presence of neoplastic cells in analyzed tissue. Possible reasons for lack of association between estimated tumor burden and percent of cells with $\mathrm{CNV}$ include acquired $\mathrm{CNV}$ in a subset of neoplastic cells, CNV in non-neoplastic cells, and selective enrichment for neoplastic nuclei during FISH. While ALK CNV likely represents a surrogate marker of neoplastic cells and has potential as a real-time quality assurance measure for ALK gene rearrangement testing, additional investigation is needed to identify the reason(s) for the lack of correlation observed between the pathologist-estimated tumor burden and the proportion of cells with ALK CNV.

\section{Digital Image Analysis as an Adjunct in the Analysis of Her2 Neu Staining of Breast Biopsies}

BJ Platt, TA Summers, JM Wells, R Barner. Walter Reed National Military Medical Center, Bethesda, MD.

Background: Clinically relevant immunohistochemical (IHC) stains are used to assist pathologists in determining susceptibility to targeted therapies in patients diagnosed with cancer, and comprise those stains that markedly impact patient care with prolonged courses of expensive, highly-effective, and targeted medications. However, interpretations of IHC staining parameters can be subjective and are prone to inter- and intra-observer variability. Virtual microscopy digitizes glass slides, creating digital image environments for subsequent computer assisted interpretation of pathologic information. Design: Automated HER2/neu IHC staining (HerCepTest, Dako, Carpinteria, CA) was performed on newly diagnosed, invasive breast cancers, and scored according to the current ASCO/CAP guidelines. First tier HER2/neu IHC analysis was performed manually by the staff pathologist of record, while a second tier manual review was subsequently performed by the quality control (QC) reviewer for all HER2/neu stains. All HER2/neu stains were then digitized for analysis (IHC HER2 IAAlgorithm; Aperio Scanscope XT; Vista, CA). A minimum of 1000 cells were analyzed for each case, ensuring proper representation of all staining patterns present on individual slides and only scoring invasive tumor elements. The digital IA results were then provided to the QC pathologist to aid in rendering the most accurate and informed final interpretation; however, the QC-assisted manual scores were recorded in order to compare to the digital IA scores.

Results: 191 HER2/neu stains were evaluated. Concordance between the ASCO/CAP scores (negative: $0,1+$, equivocal: $2+$, and positive: $3+$ ) for manual and digital analysis methods was $84 \%(160 / 191)$. Of the 31 discordant cases, two cases were clinically meaningful, demonstrating negative FISH results after receiving a digital score of $2+$ and a manual score of $3+$. These two cases were interpreted as equivocal in the final pathologic diagnosis.

Conclusions: The adjunctive use of automated digital IA in the evaluation of clinically pertinent IHC stains may increase the specificity and positive predictive value of interpretations by increasing objectivity and therefore helping to standardize and enhance quantification.

Disclaimer: The views expressed in this presentation are those of the author and do not necessarily reflect the official policy or position of the Department of the Army, Department of the Navy, Department of Defense, or the U.S. Government.

\section{Validation Study of Telepathology on Neuropathology Frozen} Section Diagnosis in a Multi-Hospital Pathology Department

J Poveda, W Yu, VNose, C Gomez-Fernandez. University of Miami, Miami, FL.

Background: Telepathology is increasingly used for neuropathology intraoperative frozen section (FS) with great accuracy. In our department, two rotating on-duty neuropathologists cover neuropathology FS in 3 hospitals, located about a 15-minute walking distance from each other. When the neuropathologists cannot be physically available, a general surgical pathologist covers the neuropathology FS. Telepathology has the great potential to improve the turnaround time (TAT), in particular when there are simultaneous FS in different hospitals. Additionally, telepathology can facilitate consultation and improve patient care. We explored the feasibility of implementing dynamic telepathology for FS in our department.

Design: Our telepathology system includes an Olympus BX40 microscope, an Olympus DP71 camera and MicroSuite Pathology Edition software in the FS suite. Access to the internet and internet browser are the only requirements for the pathologist's computer terminal. For this study, 10 FS cases were randomly chosen by an assistant. The slides were transmitted in real time to 4 pathologists ( 2 neuropathologists and 2 general surgical pathologists) by a junior resident. Short patient history and specimen site were provided. The pathologists independently evaluated each FS slide and rendered a telepathology diagnosis (TPD). Afterward, the glass slides were reviewed and each gave a light microscope diagnosis (LMD). Neither the resident nor the pathologists knew the original FS diagnoses (FSD) or the permanent section diagnoses (PSD). There were no discrepancies between the FSD and PSD.

Results: There were 40 TPD and LMD diagnoses among the four pathologists. The overall TPD accuracy was $92.5 \%$; the LMD accuracy was $97.5 \%$. For 20 of the 40 TPD responses, the diagnoses were made by neuropathologists with a TPD accuracy of $95 \%$ and LMD accuracy of $100 \%$. The general surgical pathologists TPD accuracy was $90 \%$ and an LMD accuracy of $95 \%$. Additionally, the diagnoses rendered by the neuropathologists were quicker and more specific.

Conclusions: The diagnostic accuracy of telepathology for neuropathology FS is very similar to the conventional light microscope method. There was no overall difference between TPD and LMD. Furthermore, the neuropathologists were quicker and more specific in their diagnoses, probably related to their expertise. Telepathology is an excellent tool for the practicing pathologist that can facilitate consultation, improve turn around time, and optimize patient care.

\section{Dissecting Errors in the Histology Laboratory}

SM Pyatibrat, JD Goldsmith, SS Raab, YB Kushner. Beth Israel Deaconess Medical Center, Boston, MA; Eastern Health Authority, St-John's, Newfoundland, Canada.

Background: Previous studies have examined error and the role of quality improvement in surgical pathology. Although various steps of specimen processing have been assessed, there is variation in the reported rates of defects. Methods of data collection regarding error are not universal and make current data difficult to replicate at other institutions. Little data exist on vulnerable steps in the histology laboratory workflow. We undertook a study of occurring errors in the histology laboratory in order to dissect their nature and causes.

Design: In this study, we evaluate errors in the histology laboratory at a major academic center with an annual surgical specimen volume of approximately 60,000 . Errors were defined as labeling incidents or processes that may have resulted in significant patient harm if they were not corrected prior to reaching the patient. We created a numerical step key (NSK) identifying all steps in the histology laboratory workflow from accessioning to slide pick-up by the pathologist. An error log was created using the NSK to document vulnerable steps and timing of error capture, along with date/time of error, original accession number, error description, action taken, re-work time, and whether the error was internal (caught within the laboratory) or external (released to the pathologist). Notably, an error at a single step was counted only once, omitting the "domino" effect of carry-over errors. The data were collected utilizing self-reporting by technicians, laboratory system administrators, residents, and pathologists.

Results: The data were collected over a 5 month period, with a total case number of 24324 . The histology workflow NSK included 53 steps. The error rate was $1.0 \%$ $\pm 0.3 \%$ (244 errors in total). All errors were subdivided into internal and external to the laboratory. Internal errors accounted for $81 \%$ of all defects. The majority of errors $(76 \%)$ were attributed to transcription, whereas cutting and pick-up errors accounted for $21 \%$. On average, histology staff spent 6.4 minutes $(\min =1, \max =120)$ rectifying a single error, which resulted in nearly 26 hours of wasted time over the study period. Conclusions: Errors are surprisingly frequent occurrences in the histology laboratory. They are a major source of liability for clinically important diagnostic errors and have the potential for serious adverse events and patient harm. This study dissects types, locations, and rates of error in order to target specific vulnerable steps amenable to process change and quality improvement.

\section{Rapid On-Site Cytologic Evaluation of CT-Guided Lung} Biopsies: An Inter-Institutional Comparison of Two Academic Medical Centers

KP Rao, KJ Brister, PA VanderLaan, HH Wang, JD Marotti. Dartmouth-Hitchcock Medical Center, Lebanon, NH; Beth Israel Deaconess Medical Center, Boston, MA. Background: Institutions vary in their methods and utilization of rapid on-site cytologic evaluation (ROSE). The purpose of this study was to compare ROSE techniques and outcomes for CT-guided lung biopsies at two academic medical centers.

Design: A retrospective review of all lung CT-guided FNA and/or core-biopsies with touch preparations between August 1,2011 and August 1, 2012 was performed at two major academic medical centers. Following IRB approval, departmental data bases and electronic medical records were reviewed to record number of procedures with and without ROSE, procedure time, number of repeat procedures, number of passes, presence of complications, and distribution of ROSE and final diagnoses. 
Results:

\begin{tabular}{|c|c|c|c|}
\hline Variable & Institution & & P value \\
\hline & $\mathrm{A}$ & $\mathrm{B}$ & \\
\hline Procedure \# & 187 & 90 & \\
\hline Repeat procedures & $2(2.3 \%)$ & $4(2.1 \%)$ & 1.0 \\
\hline Patient \# & 85 & 181 & \\
\hline M:F & $41: 44$ & $97: 84$ & 0.42 \\
\hline Mean age & M $67(27-88)$ & M 69 (33-91) & \\
\hline & F $65(24-89)$ & F 67(37-89) & \\
\hline Specimen type & & & $<0.001$ \\
\hline Core/TP only & $72(83 \%)$ & $190(100 \%)$ & \\
\hline FNA & $9(10 \%)$ & 0 & \\
\hline Core/TP \& FNA & $6(7 \%)$ & 0 & \\
\hline ROSE done & $84(97 \%)$ & $190(100 \%)$ & 0.01 \\
\hline Adequate on ROSE & $65(77 \%)$ & $131(69 \%)$ & 0.15 \\
\hline Average \# of passes & $2.1(1-7)$ & $5.0(1-15)$ & $<0.0001$ \\
\hline First pass that is adequate & $1.3(1-4)$ & $2.1(1-11)$ & 0.0001 \\
\hline Average \# of passes if ROSE is adequate & $2.0(1-4)$ & $5.0(1-15)$ & $<0.0001$ \\
\hline Average \# of passes when ROSE is inadequate & $2.6(1-7)$ & $4.9(1-10)$ & $<0.0001$ \\
\hline Average procedure time in minutes & $33(10-155)$ & $36(20-95)$ & 0.16 \\
\hline Complications & & & \\
\hline Hemorrhage & $3(3.5 \%)$ & $2(1.1 \%)$ & 0.17 \\
\hline Pneumothorax & $6(6.9 \%)$ & $18(9.6 \%)$ & 0.46 \\
\hline Positive rate for adequate ROSE cases & $80 \%$ & $95 \%$ & 0.001 \\
\hline Positive rate for inadequate ROSE cases & $37 \%$ & $22 \%$ & 0.20 \\
\hline Final diagnosis & & & 0.004 \\
\hline ND or negative & $19(22 \%)$ & $47(25 \%)$ & \\
\hline Atypical & $6(6.9 \%)$ & 0 & \\
\hline Suspicious & $3(3.5 \%)$ & $6(3.2 \%)$ & \\
\hline Positive & $59(68 \%)$ & $137(72 \%)$ & \\
\hline
\end{tabular}

No significant difference was noted between patients with and without complications in procedure time ( 34 vs 35 minutes, $\mathrm{P}=0.67$ ) or number of passes ( 3.7 vs $4.1, \mathrm{P}=0.38$ ). Conclusions: In both institutions, core biopsy with on site evaluation of touch preps for adequacy is preferred for CT-guided lung procedures. Higher procedure volume and increased number of passes do not appear to have a major impact on procedure time, complications, or the overall final pathologic diagnosis. However, higher procedure volume was associated with a higher positive rate for those cases deemed adequate onsite. This suggests that improved familiarity of touch prep specimens by cytology personnel might lead to improved onsite diagnostic accuracy.

\section{$2006 \quad$ Utility of Thrombophilia Testing in the Reproductive Setting}

$S$ Ravishankar, LR Wolgast, K Demasio, JH Rand. Montefiore Medical Center, Bronx, NY.

Background: The rising cost of healthcare has led to closer scrutiny of the utilization of laboratory testing. Specialized coagulation and molecular tests for thrombophilias, the average cost of which is approximately $\$ 1500$, are often ordered in the evaluation of pregnancy complications, such as repeated pregnancy losses (RPL), intrauterine fetal demise (IUFD) and pre-eclampsia. Guidelines on appropriate indications have been published by various organizations, including ACOG, ACCP, BSH and ISTH. The objective of this study was to assess the "real world" utilization of thrombophilia testing in the reproductive setting at a large, urban tertiary care academic center and compare this with published guidelines.

Design: The results of 351 nonconsecutive thrombophilia studies in the reproductive setting from 2009-2012 were reviewed and correlated to clinical indications found upon chart review. Indications were categorized as consistent or inconsistent with recommendations as defined by review of consensus guidelines in hematologic and obstetric literature, which generally limit testing to patients with RPL or a personal or family history of thromboembolism.

Results: A total of 2100 tests were reviewed, the results of which are shown in Table 1. Interestingly the likelihood of finding an abnormality was not significantly different between groups. To our knowledge this is the largest study of the use of thrombophilia testing at a single center.

\begin{tabular}{|c|c|c|c|c|c|}
\hline & Consistent $\dagger$ & & Inconsistent: & & \\
\hline & $\#$ Tested & $\# / \%$ Abnormal & $\#$ Tested & $\# / \%$ Abnormal & $\%$ Appropriate \\
\hline Antithrombin III & 97 & $2 / 2 \%$ & 90 & $3 / 3 \%$ & $52 \%$ \\
\hline Protein C & 108 & $1 / 1 \%$ & 100 & $2 / 2 \%$ & $52 \%$ \\
\hline Protein S & 117 & $32 / 27 \%$ & 110 & $34 / 31 \%$ & $52 \%$ \\
\hline Plasminogen & 15 & $0 / 0 \%$ & 9 & $0 / 0 \%$ & $63 \%$ \\
\hline $\begin{array}{l}\text { Activated Protein } \\
\text { C Resistance }\end{array}$ & 92 & $2 / 2 \%$ & 75 & $4 / 5 \%$ & $55 \%$ \\
\hline $\begin{array}{l}\text { Lupus } \\
\text { Anticoagulant }\end{array}$ & $\mid 160$ & $7 / 4 \%$ & 175 & $9 / 5 \%$ & $48 \%$ \\
\hline $\begin{array}{l}\text { Antiphospholipid } \\
\text { Antibody Screen }\end{array}$ & $\mid 124$ & $11 / 9 \%$ & 147 & $\mid 14 / 10 \%$ & $46 \%$ \\
\hline $\begin{array}{l}\text { Factor Veiden } \\
\text { Mutation }\end{array}$ & 97 & $1 / 1 \%$ & 86 & |3/3\% & $53 \%$ \\
\hline \begin{tabular}{|l|} 
Prothrombin \\
G20210A Mutation
\end{tabular} & 100 & $2 / 2 \%$ & 89 & $2 / 2 \%$ & $53 \%$ \\
\hline MTHFR Mutation & 84 & $31 / 37 \%$ & 82 & $36 / 44 \%$ & $51 \%$ \\
\hline Total & 1057 & $101 / 10 \%$ & 1043 & $123 / 12 \%$ & $50 \%$ \\
\hline
\end{tabular}

$\dagger$ Provoked DVT or PE, RPL, Family history \$Unprovoked DVT or PE, primary infertility, isolated IUFD or SAB, pre-eclampsia, IUGR, preterm labor, ectopic pregnancy, abruption, other

Conclusions: We determined that $50 \%$ of thrombophilia tests are ordered for indications that are inconsistent with published guidelines, indicating a significant mismatch between the literature and clinical setting. Pathologists need to investigate the reasons behind this mismatch and coordinate with clinicians on reviewing and modifying recommendations for appropriateness, which will likely result in major health care savings.

\section{Proposal for a New Classification of "Equivocal” Fibroepithelial} Lesions (FELs)

E Resetkova, N Sneige. University of Texas MD Anderson Cancer Center, Houston, TX. Background: Management of "equivocal" FELs with unusual histomorphology or abnormal clinical and imaging findings diagnosed by core biopsy (CB) is still controversial. These lesions are difficult to classify even upon excision and infrequently subjected to second opinion.

Design: Final excisional specimens of equivocal FELs diagnosed on CB were blindly re-reviewed by two additional experienced breast pathologists, in order to evaluate inter-observer variability and to assess importance of each individual histologic parameter for final classification. The institutional review board of The University of Texas M.D. Anderson Cancer Center approved this study. The reviewers based their diagnoses on systematic evaluation of numerous histologic criteria (predominan architectural pattern, margins, satellite nodules, stromal overgrowth, stromal cellularity and stromal atypia, mitotic activity, stromal periductal condensation and hyalinization, stromal heterogeneity, presence of heterologous components and/or fat, and degree of epithelial atypia). The inter-observer variability results were analyzed using percentage agreement and the Kappa test.

Results: Sixty five surgical cases of FELs were re-reviewed. We report a very high inter-observer variability between the original pathology diagnosis and two experienced breast pathologists. $38 \%$ of cases were upgraded either from FA to benign PT, or from benign PT to PT of uncertain malignant potential. Based on re-review, different clinical management would be recommended in $20 \%$ of cases. The most significant histologic parameters consistently yielding to an upgrade by expert pathologists were focal infiltration at the tumor periphery $(\mathrm{p}=0.01)$, presence of satellite nodules $(\mathrm{p}=0.05)$, and stromal heterogeneity $(\mathrm{p}=0.05)$.

Conclusions: Due to the difficulty to reach consensus on diagnosis of equivocal FELs and a high inter-observer variability, we propose a new classification and recommend an algorithm for their clinical management.

2008 Previously Stained Slides for Use with Immunohistochemistry: A Single Institution Study for Consult Cases and Small Biopsies

MD Rumery, DJ Davis, S Lucia. University of Colorado, Aurora, CO.

Background: Limited tissue is often available for the use of immunohistochemistry (IHC) in the case of small biopsies, where the block may be easily exhausted. In such instances, special stains may have been prepared that are of limited value relative to a desired IHC marker. While de-staining of prior stained slides can be used in these cases, the optimal stains for use and the prior stain's effect on signal intensity have not been previously reported. Here we report a semi-quantitative analysis of IHC intensity when performed on previously stained and de-stained slides.

Design: Using archival tissue, we stained slides with commonly used special stains for a number of disciplines often affected by limited tissue including neuropathology, hepatopathology, hematopathology, breast, and cytopathology. The stains used included negative IHC controls, hematoxylin and eosin (H\&E), iron, trichrome, acid fast bacilli, periodic acid Schiff (PAS), PAS with diastase (PAS/D), Congo Red, and PAS Orange $G$. These previously stained slides then had the coverslip removed and were de-stained using routine methods. Subsequent to de-staining, the following IHC was performed: AE-1/AE-3, CK 7, CK 20, IDH-1, Mib-1, synaptophysin, CD 3, CD 20, CD 34, bcl-2, bcl-6, WT-1, BerEp4, ER, and PR. For each stain, a 'gold standard' control was prepared using our institution's procedure for IHC. The intensity of IHC signal for each de-stained slide was then compared to this 'gold standard' and given a semi-quantitative score $(0-3+)$.

Results: Nine special stains and 15 antibodies were evaluated in total. Significant variation in IHC signal intensity was observed between both stains and specific antibodies. Despite the variation, some stains persistently resulted in stronger IHC signal intensity. Slides de-stained from trichrome, negative IHC controls, and H\&E resulted in the strongest staining intensity on average. PAS/D resulted in the least intense signal, and the staining that was present showed a loss of specificity.

Conclusions: De-staining trichrome, negative IHC control, and H\&E stains with subsequent IHC resulted in the strongest signal with varying results of other stains tested. We hypothesize that the amount of chemical alteration of the protein moieties caused by the stain impacts the staining intensity, as demonstrated by the poor intensity and specificity of the IHC on PAS and PAS/D. Details of staining and de-staining procedures will be discussed.

2009 "Urgent" Surgical Pathology Specimens; a Review and Comparison with Critical Values in Anatomical Pathology

CM Ryan, BM Loftus. Tallaght Hospital, Dublin, Ireland.

Background: What is designated an urgent diagnosis in surgical pathology is highly subjective and open to interpretation from pathologists and clinicians. Attempts have been made to formalize the definition by the Association of Directors of Anatomic and Surgical Pathology (ADASP) with the publication of examples of critical diagnoses requiring verbal communication. In our practice it was observed that requests designated as "urgent" by clinicians were increasing. As local policy is to prioritize "urgent" specimens and phone the report to the clinician, it was felt that the increased number of "urgents" put an undue demand on resources. It was often unclear as to why the a specimen merited urgent processing therefore a review was undertaken to assess "urgent" specimens and comparison made with ADASP guidelines to assess which specimens warranted verbal communication.

Design: A review was performed of "urgent" specimens over six-months from $1^{\text {st }}$ July to $31^{\text {st }}$ December 2011. "Urgent" histological specimens were retrieved from a search on Winpath with a simultaneous search for SNOMED, quality and procedure codes. The turn around time (TAT) of these specimens was assessed and our standards of practice 
compared with international guidelines, which recommend a two-day TAT for urgent biopsies ( $80 \%$ threshold). A subset of these "urgent" requests was compared to ADASP examples of critical diagnoses.

Results: $4.23 \%$ of specimens ( $\mathrm{n}=587$ ) were labeled "urgent". $74.7 \%$ were biopsies, $23 \%$ malignant \& $31 \%$ verbally communicated $(\mathrm{n}=183)$. Only $2.2 \%$ of requests $(\mathrm{n}=4)$ would require verbal communication as per ADASP guidelines on critical diagnoses [unexpected malignancies $(\mathrm{n}=2)$, acid fast bacilli $(\mathrm{n}=1)$, virus in an immunocompromised patient ( $\mathrm{n}=1)$ ]. TAT for all "urgent" specimens was $44 \%$ at two days. If was felt that "urgent" specimens directly communicated to the clinician were a truer reflection of urgent status. The TAT for this group was $65.5 \%$ at 2 days ( $69 \%$ for biopsies).

Conclusions: Differences exist between what pathologists and clinicians consider as urgent specimens. Inappropriate designation of some specimens as "urgent" may result in increased TAT and substandard compliance with international quality guidelines. Specimens designated as clinically urgent in surgical pathology do not necessarily equate to critical diagnoses requiring verbal communication to clinicians. We recommend that each institution compile a list of example critical diagnoses that require verbal communication and educate clinical teams as to appropriateness of designating specimens urgent.

2010 Characterization of EWSR1 Break Apart FISH in Non-EWSR1 Related Samples: Avoiding the False Positive Pitfall

N Sadri, PJ Zhang. University of Pennsylvania, Philadelphia, PA.

Background: Hybridizing two colored DNA probes flanking break points of a specific gene can visualize a signal separation (SS) in tumor cells containing that translocation. This break-apart FISH assay is widely used on routine paraffin section for diagnosis Currently there lacks standardization in interpreting the presence and incidence of SS Although false positive SS are recognized to exist their characterization and incidence has not been sufficiently characterized.

Design: We performed EWSRI break-apart probes (Vysis) on sarcomas with a nonEWSR I translocations (synovisal sarcomas [SYS; $\mathrm{n}=3$ ] and alveolar rhabdomyosarcomas [AR, $\mathrm{n}=4]$ ]), 3 breast carcinomas (BRCa) and 2 normal tonsils. Tumors known to have EWSRI translocation (EWSRI+; 1 DSRCT, 3 EWS/PNET, 1 extraskeletal myxoid chondrosarcoma) were evaluated as a control. Each tumor cell was categorized to have: at least two intact alleles (IA); at least one IA with one SS; or unpaired signal(s) (UPS). Cells with no signal, only one unpaired signal, or only one intact allele were not counted. The average EWSRI alleles per cell were determined. The signal width (SW) was calculated for SS.

Results: See table below.

\begin{tabular}{|l|l||l|l|l|l|l|l||}
\hline Diagnosis & IA & UPS & SS & 1 SW & >2 SW & $>$ 3 SW & $>$ 4 SW \\
\hline STS/AR $(\mathrm{n}=7)$ & $49 \pm 18$ & $24 \pm 14$ & $21 \pm 5$ & $12 \pm 3$ & $9 \pm 4$ & $4 \pm 3$ & $0.7 \pm 0.6$ \\
\hline EWSR $1+(\mathrm{n}=5)$ & $3.0 \pm 2.7$ & $30 \pm 10$ & $67 \pm 12$ & $9 \pm 4$ & $58 \pm 14$ & $47 \pm 10$ & $29 \pm 12$ \\
\hline BRCa $(\mathrm{n}=3)$ & $49 \pm 10$ & $23 \pm 8$ & $29 \pm 12$ & $12 \pm 1$ & $29 \pm 9$ & $10 \pm 7$ & $3 \pm 2$ \\
\hline Tonsil $(\mathrm{n}=2)$ & $55 \pm 4$ & $30 \pm 1$ & $18 \pm 3$ & $8 \pm 1$ & $11 \pm 3$ & $7 \pm 4$ & $2 \pm 1$ \\
\hline
\end{tabular}

All values provided $\%$ of cells reported as mean $+/$ - std.

Conclusions: A range of SS can be seen in all non-EWSR1 related samples. Given its similar frequency in all groups analyzed, 1 SW SS is likely a nonspecific finding. In non-EWSR 1 related samples when using 2, 3 or $4 \mathrm{SW}$ as cut off criteria for SS abnormal signals could be found in up to $40 \%, 15 \%$ or $5 \%$ of the total countable cells respectively. However, in this group $50 \%$ of countable cells contained IA as compared to $3 \%$ in EWSR 1+ tumors. Aneuploid EWSRI signal was common in SS and AR, but not in EWSRI+ tumors, the significance of which is unknown. UPS are likely due to artifact owing to nuclear truncation as it remains constant across all groups. The observed SS in non-EWSR 1 related tissue is likely an artifact related to assay procedure and tissue processing, and less likely due to tumor specific nuclear changes, as they also occur in non-tumor tissue. In addition to focusing on the width of SS and \% of cells with SS, evaluating tumor cells with IA might help to recognize a false SS result.

2011 Discrepancies between Intraoperative Consultations and Final Pathology Report: Root Cause Analysis as a Means for Performance Improvement

SB Sams, JA Wisell. University of Colorado School of Medicine, Aurora, CO.

Background: Discrepancies between intraoperative consultation with frozen section diagnosis and the final pathology report potentially alter surgical decisions and impacts patient care. Our goal was to use root cause analysis to identify the source of discrepancy and guide performance improvement initiatives.

Design: A retrospective review of intraoperative consultations $(n=1042)$ performed at a tertiary level hospital and cancer center over a six month period was performed to identify deferrals and discrepancies between the intraoperative consult report and the final pathology report. Using a modified Eindhoven classification method we identified the process errors and cognitive errors leading to discrepant results.

Results: A total of $50(4.8 \%)$ discrepancies and $24(2.3 \%)$ deferrals were identified and in all cases, no major clinical harm was identified. Process errors $(n=28,56 \%)$ were subdivided into gross sampling $(\mathrm{n}=16,32 \%)$ histologic sampling $(\mathrm{n}=8,16 \%)$ and surgical sampling $(n=2,4 \%)$. Interpretation errors $(n=22,44 \%)$ included under-calls false negative $(n=8,16 \%)$ over-calls/false positive $(n=10,20 \%)$ and misclassification errors $(n=4,8 \%)$.

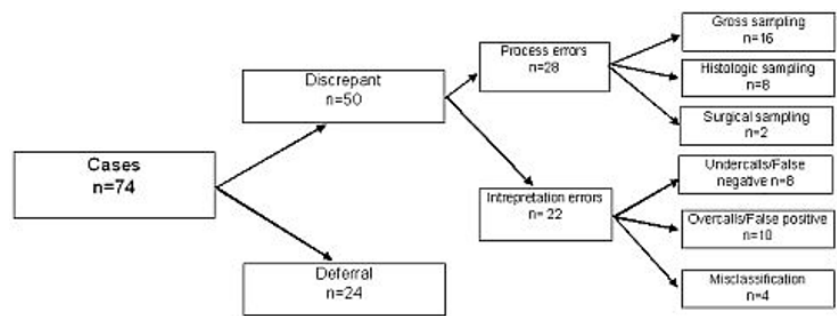

Conclusions: The majority of discrepancies $(96 \%)$ occurred during gross room processing or interpretation and only small percentage were pre-analytic (due to surgical sampling). In our limited study, the area for greatest improvement would be directed towards gross sampling of specimens followed by a standardized approach to frozen section histology. Interpretation errors may be secondary to heuristic bias or educationa limitiations. We conclude that analysis of the etiology of discrepancies can be a useful tool to identify areas for performance improvement.

\section{Continuous Quality Improvement: Procedural Standardization} of Intra-Operative Consultation Coding in Surgical Pathology

$S$ Sayeed, T Lea, CN Powers, MO Idowu. Virginia Commonwealth University, Richmond, VA.

Background: Continuous quality improvement is necessary not only to prevent preanalytic and diagnostic errors, but also to ensure accurate CPT coding. For intraoperative consultations (IC) accurate coding relies upon clear documentation of the procedure (frozen[FS] section, touch preparation[TP] or both), number of specimen(s) and number of ICs performed on each specimen (first FS, second FS, etc). The purpose of this study was to evaluate and improve our documentation system for ICs to minimize coding and billing errors.

Design: Documentation and coding of all ICs over a 3 month period in 2010 prior to procedural standardization (PS) was reviewed, by correlating entries in the IC logbook with documentation in final pathology reports and CPT codes entered in the Cerner Laboratory Information System (LIS). We then instituted PS requiring 1) dictation of specific IC procedure using templates based on CPT terminology, 2) coding for IC at the time of dictation, 3) daily correlation of IC logbook entries with CPT codes in the LIS. We compared a three month period 18 months following implementation of PS. Results: There were 334 specimens with IC reviewed during the pre-PS period in 2010 , of which $105(31 \%)$ were incorrectly coded. The improper coding was due to insufficient documentation and inconsistent use of CPT terminology. Following implementation of PS, of the 405 specimens reviewed, 31 (8\%) were incorrectly coded (Table 1). Incorrect coding leading to revenue loss was primarily due to either absent codes or under-coding (Table 2). Reimbursement was issued for cases with over-coding. Coding errors more commonly occurred with complex specimens where TP and FS were utilized.

Conclusions: Significant improvement in IC coding followed implementation of PS. PS and continuous monitoring is important for minimizing / eliminating miscoding errors. Education of staff, residents and faculty is essential. Laboratories should consider PS with continuous monitoring to minimize errors.

\begin{tabular}{|c|c|c|c|c|}
\hline & \multicolumn{2}{|c|}{ Pre Procedural Standardardization } & \multicolumn{2}{|c|}{ Post Procedural Standardization } \\
\hline & 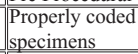 & $\begin{array}{l}\text { Improperly coded } \\
\text { specimens }\end{array}$ & $\begin{array}{l}\text { Properly coded } \\
\text { specimens }\end{array}$ & $\begin{array}{l}\text { Improperly coded } \\
\text { specimens }\end{array}$ \\
\hline \multicolumn{5}{|c|}{ (1) } \\
\hline FS & $83 \%$ & $17 \%$ & $94 \%$ & $6 \%$ \\
\hline TP & $62 \%$ & $36 \%$ & $97 \%$ & $3 \%$ \\
\hline FS\&P & $6 \%$ & $96 \%$ & $76 \%$ & $24 \%$ \\
\hline Additional FS & $40 \%$ & $60 \%$ & $67 \%$ & $33 \%$ \\
\hline Gross only & $50 \%$ & $50 \%$ & $89 \%$ & $11 \%$ \\
\hline Total & $69 \%$ & $31 \%$ & $92 \%$ & $8 \%$ \\
\hline
\end{tabular}

Table 2. Coding Errors
\begin{tabular}{|l||l||l|}
\hline \hline & Pre PS $(\mathrm{n}=105)$ & Post PS $(\mathrm{n}=31)$ \\
\hline No code & $56(53.3 \%)$ & $10(32 \%)$ \\
\hline Under-coding & $34(32.4 \%)$ & $14(45 \%)$ \\
\hline Over-coding & $15(14.3 \%)$ & $7(23 \%)$ \\
\hline Extrapolated Revenue loss for the year using Medicare Expect & $\$ 31,702$ & $\$ 3,821$ \\
\hline
\end{tabular}

2013 Malignant Colonic Polyps: Diagnostic Accuracy and Quality of Surgical Pathology Reports

EL Schollenberg, W-Y Huang, HL Sapp. Queen Elizabeth II Health Centre \& Dalhousie University, Halifax, NS, Canada.

Background: The goal of population-based colon cancer screening programs, such as the one recently launched in our province, is to diagnose and treat more neoplastic lesions at a pre-invasive or early invasive stage. Pathologists can therefore expect to encounter more so-called "malignant polyps," defined as adenomatous polyps in which malignant glands invade into submucosa. The appropriate management of these patients depends on the accuracy and completeness of the surgical pathology report. The goal of the present study was to review a set of potentially problematic malignant polyps in order to determine 1) the completeness and accuracy of the original reports, and 2) the incidence and characteristics of false-positive reports.

Design: Diagnoses of non-rectal colonic adenocarcinoma initially made on endoscopic mucosal biopsies were collected, representing 762 cases over a period of 11 years. 564 biopsies $(74 \%)$ were followed up by colectomy in our institution. Of these, 32 cases $(6 \%$ of 564) of biopsy-diagnosed invasive carcinoma were followed up with no evidence of 
malignancy on resection specimens. The biopsy slides for these cases were reviewed by two gastrointestinal pathologists, who classified each case as either malignant (true invasion) or benign (pseudo-invasion).

Results: Of the reviewed biopsies, 24 (75\%) were deemed to represent true malignant polyps. The original reports on half of these ( 12 cases) were incomplete. Of the remaining 8 problematic cases, 6 were biopsies from the left colon. 3 cases were reclassified on retrospective review as false positive diagnoses (representing $0.5 \%$ of the 564 patients who underwent colectomy). In 5 cases, the two reviewing pathologists were unable to reach a consensus diagnosis on the presence or absence of invasion.

Conclusions: In this series, most diagnoses of malignant polyps were accurate, although a significant number of reports lacked complete information necessary to guide management. The diagnosis of early invasion in this context can be challenging, and surgical pathologists need to be aware of the diagnostic pitfalls. There is a need to develop ancillary tests for resolving diagnostic dilemmas.

\section{High Factor VIII Can Falsely Increase Factor IX and XI Levels Due to the Shortening of aPTT}

R Shafi, V Kostousov, K Nguyen, S-KR Hui, J Teruya. Baylor College of Medicine, Houston, TX.

Background: The acute phase response is followed by an elevation of proteins in the plasma, including coagulation factor VIII (FVIII) levels. High FVIII levels cause a shortening of the activated partial thromboplastin time (aPTT), and may also affect the measurement of aPTT-based coagulation factor levels, including those of factors IX (FIX) and XI (FXI). This may mask a deficiency of these factors, a situation which can lead to hazardous bleeding risks. The purpose of this study is to determine if high FVIII levels will affect the measurement of FIX and FXI during the acute phase response. Design: Testing samples with mild deficiency were prepared from citrated FIX and FXI deficient plasmas mixed with normal pooled plasma. Then recombinant FVIII was added in order to increase FVIII activity by $100 ; 300$ and $500 \%$ from baseline. FIX, FXI, FVIII and aPTT were measured by a coagulation analyzer using commercially available reagents. All experiments and measurements were run in triplicate and data are reported as mean \pm SD. Statistical analysis was performed using ANOVA test and significance was set up at $\mathrm{p}<0.05$.

Results: Elevated FVIII activity in plasma significantly shortened aPTT. Although the FIX and FXI levels were not significantly changed, the FIX level was overestimated by $1 \%$, and FXI level by $0.8 \%$ in average with each $+100 \%$ of FVIII increment.

\begin{tabular}{|c|c|c|c|}
\hline Factor VIII Levels & FVIII,\% & FIX,\% & aPTT,sec \\
\hline Baseline Level & $104+/-7$ & $20+/-6$ & $36.3+/-1.7$ \\
\hline$+100 \%$ & $199+/-7$ & $21+/-5$ & $33.5+/-1.3$ \\
\hline$+300 \%$ & $428+/-7$ & $24+/-6$ & $31.0+/-1.2$ \\
\hline$+500 \%$ & $655+/-33$ & $26+/-4$ & $29.5+/-1.2$ \\
\hline $\mathrm{p}$-value & N/A & 0.50 & 0.0013 \\
\hline
\end{tabular}

Factor XI Deficient Plasma

\begin{tabular}{|l||l||l|l|}
\hline Factor VIII Levels & FVIII, \% & FXI, \% & aPTT,sec \\
\hline Baseline Level & $119+/-4$ & $15+/-4$ & $37.5+/-1.7$ \\
\hline$+100 \%$ & $212+/-7$ & $15+/-4$ & $34.7+/-1.4$ \\
\hline$+300 \%$ & $415+/-24$ & $17+/-3$ & $31.7+/-1.7$ \\
\hline$+500 \%$ & 630+/-23 & $19+/-3$ & $30.5+/-1.5$ \\
\hline p-value & N/A & 0.51 & 0.0027 \\
\hline
\end{tabular}

Conclusions: Elevated FVIII levels cause a meaningful shortening of the aPTT that is associated with a mild increase of FIX and FXI activity. Further studies are warranted to evaluate if high FVIII levels compromise these factor activity measurements in cases of severe factor deficiency.

\section{Routine Use of p16 Aids the Detection of High Grade Squamous} Intraepithelial Lesions in Endocervical Curettage Specimens

AA Shah, SK Jeffus, Z Zhao, H Scruggs, EB Stelow. University of Virginia, Charlottesville, VA.

Background: The utility of endocervical curettage (ECC) in the diagnosis of cervical neoplasia is controversial as studies have shown that it is fraught with false positive and negative results as well as significant diagnostic interobserver variability. p16 has been shown to be useful in detection of cervical high grade squamous intraepithelial lesions (HSIL). We questioned whether p16 staining of ECCs in patients with antecedent ASC-H and HSIL Pap test would improve its diagnostic sensitivity.

Design: 58 consecutive ECCs that were previously diagnosed as negative for SIL in patients with antecedent ASC-H or HSIL Pap tests were retrieved. An H\&E recut and immunohistochemistry for 16 were performed on all cases. A diagnosis of HSIL (CIN2/CIN3) was made if there was strong nuclear and cytoplasmic staining in cells with the appropriate cytomorphologic features. Follow-up results from Pap tests, biopsy procedures and HPV status were documented.

Results: HSIL was found in 20 ECCs $(34 \%)$ originally interpreted as negative. Of these 20 cases, 9 had moderate-sized fragments of ectocervical epithelium with HSIL that was only seen on the recut H\&E with supporting strong immunostaining with antibodies to 16 . 11 cases had rare to occasional clusters or single atypical cells with strong immunoreactivity for p16 (Figure 1).

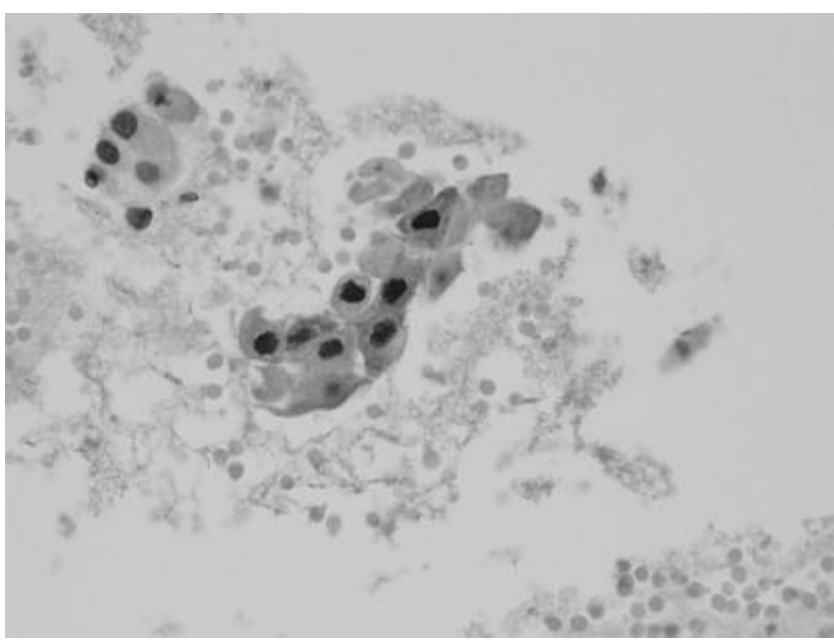

The 38 negative ECCs were either completely negative for p16 or demonstrated focal staining of non-dysplastic epithelium (e.g. tubal metaplasia). When available, HPV status for ECCs with HSIL was as follows: 8 positive, 2 negative. HPV status for negative ECCs was as follows: 15 positive, 11 negative. Follow-up was available for 26 patients and is summarized in Table 1 .

\begin{tabular}{|l|l|l||}
\hline FOLLOW UP & ECC with HSIL & ECC NEGATIVE for HSIL \\
\hline Pap Test & & \\
\hline Negative & 4 & 6 \\
\hline ASC & 2 & 3 \\
\hline LSIL & 1 & 0 \\
\hline ASC-H & 1 & 1 \\
\hline HSIL & 1 & 1 \\
\hline Biopsy/LEEP & 0 & 3 \\
\hline Negative & 2 & 0 \\
\hline LSIL (CIN I) & 8 & 0 \\
\hline HSIL (CIN II/III) & 24 \\
\hline Not available & \\
\hline
\end{tabular}

Table 1. Clinical Follow up

Conclusions: Recut H\&E and adjunct p16 immunostaining was valuable for detecting HSIL in $34 \%$ of ECCs that were previously diagnosed as negative for SIL. It may be of assistance for triaging patients who undergo ECC and have previously had ASC-H or HSIL Pap tests.

\section{Does On-Site Evaluation Improve the Diagnostic Yield of Fine} Needle Aspiration Specimens?

$W$ Shi, PG Hwang, $N$ Vergara, GH Yu, $Z W$ Baloch. Hospital of the University of Pennsylvania, Philadelphia, PA.

Background: Fine-needle aspiration (FNA) has proven to be a safe, economical, accurate, and rapid diagnostic technique. Rapid on-site evaluation (ROSE) of FNA samples has been advocated as a guide for assessing the adequacy of FNA samples and to provide an effective triaging mechanism for additional studies. The intention of this study was to explore whether ROSE is effective in procuring adequate specimen for ancillary studies and improving the final diagnostic yield.

Design: The computerized pathology files at our institution were searched for all FNA cases performed between July 2011 and December 2011; the thyroid FNA cases were excluded as ROSE is performed in $\geq 99 \%$ of these cases. The collected data points included:location of site(s) sampled, on-site evaluation performed, preliminary onsite diagnosis rendered, final cytologic diagnosis, cell blocks, and ancillary studies performed.

Results: A total of 554 FNA specimens were reviewed and included in the study; of these $56(10 \%)$ were lung FNA, $387(70 \%)$ lymph nodes, $51(9 \%)$ pancreas and 60 $(11 \%)$ were from miscellaneous sites. ROSE was performed in 300 of $554(54 \%)$ cases. A final diagnosis of malignancy was rendered in 137 of 300 (46\%) and 48 of 254 (19\%) cases with and without ROSE respectively. Adequate material for cell blocks and/or any necessary ancillary studies was obtained in 200 of 300 (61\%) cases with ROSE (cell blocks 171, immunostains 51, flow cytometry 31 ; special stains 1 , Molecular studies 13) and 201 of 254 (79\%) cases without ROSE (cell blocks 197, immunostains 18 , flow cytometry 4 ; special stains 4 ; molecular studies 3 ). On final review, 5 of 300 ROSE cases $(2 \%)$ and 5 of 254 non-ROSE cases $(2 \%)$ were non-diagnostic due to the lack of adequate specimen. Among the 29 cases considered inadequate by ROSE (29/300,10\%), only 4 were deemed non-diagnostic on final review; 1 case considered as adequate on ROSE was found to be non-diagnostic on final review.

Conclusions: ROSE can be beneficial in providing a preliminary diagnosis in cases with high clinical suspicion for malignancy which may lead to immediate management decisions; however, in cases with low clinical suspicion for malignancy it may be of minimal or no value in providing specimen adequacy assessment and improving diagnostic yield. 
2017 Effect of the Duration of Cold Ischemic Time and Temperature Conditions on Maintenance of RNA Integrity Number (RIN) in Biospecimens G Sica, C Butler, G Zhang, S Maithel, M Rossi, T Owonikoko. Emory University, Atlanta, GA.

Background: The RIN determines the integrity of extracted RNA by analyzing RNA using a combination of micropapillary electrophoresis and fluorescence detection that is analyzed using an established software algorithm. The RIN is a numerical scale ranging from 1 (degraded) to 10 (intact). A RIN $>7$ is considered sufficiently intact for most applications and is affected by ischemic time. Ischemic time can be separated into two components, warm ischemic time (surgical incision to specimen removal) and cold ischemic time (resection to tissue stabilization e.g. freezing). Previous reports have indicated conflicting results on the effect of cold ischemic time on RIN and whether temporary storage of specimens on ice prior to freezing results in improved RIN.

Design: Resected human pancreatic tissues were stored at $4^{\circ} \mathrm{C}$ prior to tissue stabilization. For the murine xenotransplant studies, athymic nude mice bearing small cell lung carcinoma (SCLC) xenografts were sacrificed by cervical dislocation at day 14 post inoculation when tumor volume averaged $800 \mathrm{~mm}^{3}$. Tumors were collected and dissected into $2 \mathrm{~mm}$ pieces and either frozen immediately, incubated at room temperature or placed on ice for the indicated time period prior to freezing. All samples were snap frozen in liquid nitrogen and stored at $-80^{\circ} \mathrm{C}$. Total RNA was extracted using miRNeasy kit, concentration was determined using a Nanodrop spectrophotometer and RNA was analyzed using an Agilent 2100 bioanalyzer Nano LapChip.

Results: The RIN for pancreas tissues ranged from 1.7 to 8.1 and did not correlate with the length of time the tissue was stored at $4^{\circ} \mathrm{C}$ prior to freezing.

Table 1. Pancreas Tissue

\begin{tabular}{||l||l|l||}
\hline Specimen & Time to Stabilization & RIN \\
\hline 1 & 45 minutes & 1.7 \\
\hline 2 & 2.5 hours & 3.1 \\
\hline 3 & 6 hours & 8.1 \\
\hline 4 & 12 hours & 5.6 \\
\hline 5 & 12 hours & 2.3 \\
\hline
\end{tabular}

In the harvested xenografted SCLC tissue, RIN ranged from 8.4 to 10 with no differences identified between tissues immediately snap frozen, stored at room temperature or on ice prior to long term freezing.

Table 2. Small Cell Carcinoma Xenotransplant RIN Values When Incubated on Ice or Room Temperature (RT)

\begin{tabular}{||l|l|l|l|l||}
\hline \multicolumn{2}{l|}{} & RIN - mouse 1 & RIN - mouse 2 \\
\hline Time to Stabilization (Minutes) & Ice & RT & Ice & RT \\
\hline 0 & 10 & & 10 & \\
\hline 15 & 10 & 10 & 9.8 & 9.6 \\
\hline 150 & 10 & 10 & 9.3 & 9.6 \\
\hline 60 & 9.9 & 9.7 & 9.8 & 8.4 \\
\hline \hline
\end{tabular}

Conclusions: Cold ischemic time up to 60 minutes at room temperature or on ice did not impact RIN values. Our result suggests that this factor may not be the main preanalytic variable determining the RIN of fresh frozen tissues. Previous recommendations for stabilization of pancreas tissues on ice or at $4^{\circ} \mathrm{C}$ prior to freezing was partly supported by our results.

2018 Role of Magnification in Visual Evaluation of Manual and Automated HPV In-Situ Hybridization in Cell Blocks (CB) from Metastatic Oropharyngeal Carcinoma

MT Siddiqui, C Cohen, N Fatima. Emory University Hospital, Atlanta, GA.

Background: Determination of Human papillomavirus (HPV) status has strong diagnostic, prognostic, and therapeutic implications in oropharyngeal cancers (OC). Determining the integration status of HPV by in situ hybridization (ISH) is cost effective and routinely utilized in clinical practice. However, the reliability of reporting is dependent on individual expertise and visual evaluation. In this study, we have compared the role of magnification in interpreting HPV ISH results of manual versus an automated method, using the Dako and the Enzo probe respectively.

Design: We evaluated $41 \mathrm{CB}$ from fine needle aspiration of metastatic OC to neck lymph nodes. We performed manual HPV ISH with the Dako probe, and Leica Bond-III automation using the Enzo probe. Punctate dot-like nuclear positivity observed with low, intermediate or high magnifications, $10 \mathrm{x}, 20 \mathrm{x}$ or $40-60 \mathrm{x}$, was assigned as $3+$, $2+$ and $1+$ respectively. A single cell showing nuclear punctate dot-like staining was considered a positive result.

Results: Two of 41 CB (4\%) with manual HPV ISH and 12 of 41 CB (29\%) with the automated method positive at 10x magnification, were considered $3+$. Fourteen of 41 CB $(34 \%)$ with manual HPV ISH and 3 of $41 \mathrm{CB}(7 \%)$ with automation positive at 20x magnification, were $2+$. Nine of $41 \mathrm{CB}(21 \%)$ with manual HPV ISH and 15 of $41 \mathrm{CB}(36 \%)$ with automation positive at 40-60x magnification, were considered $1+$.

([table1])

\begin{tabular}{|l||l||l|l|}
\hline HPV ISH (Positive) & $10 \mathrm{X}(3+)$ & $20 \mathrm{X}(2+)$ & $40-60 \mathrm{X}(1+)$ \\
\hline Manual & $2 / 41(4 \%)$ & $14 / 41(34 \%)$ & $9 / 41(21 \%)$ \\
\hline Automated & $12 / 41(29 \%)$ & $3 / 41(7 \%)$ & $15 / 41(36 \%)$ \\
\hline
\end{tabular}

Conclusions: The dot like punctate staining may be very focal which requires careful visual determination of all tumor cells. Low magnification $(10 \mathrm{x})$ reveals positivity in $4 \%$ of cases with the manual method, whereas the automated technique, being more sensitive, reveals a higher positivity rate $(29 \%)$. Hence, automation provides a better and more efficient method for evaluating HPV ISH in cases of metastatic OC. Failure to evaluate under high magnification (40-60x) however, could give false negative results in $21 \%$ of cases with the manual method and $36 \%$ with automation. This could confound the analysis and effect therapeutic decisions. The authors conclude that magnification power plays a critical role in visual evaluation of HPV ISH. Additionally, automated technique of HPV ISH evaluation provided better quality assaurance for this type of testing in their laboratory.
2019 Assessment of Cognitive Bias as a Root Cause of Diagnostic Interpretation Error in Pathology Residents

ML Smith, S Dintzis, SS Raab. Mayo Clinic Arizona, Scottsdale, AZ; University of Washington, Seattle, WA; Eastern Health and Memorial University of Newfoundland, St. John's, NL, Canada.

Background: The cognitive root causes of diagnostic interpretation error in surgical pathology are poorly understood. We developed a simulation-based medical education (SBME) model to measure the biases associated with resident diagnostic interpretation error and developed methods of reference range forecasting for residents and pathologists to reduce error frequency.

Design: We measured diagnostic interpretation errors in a cohort of 8 residents participating SBME, designed incrementally to challenge residents with more difficult and rare cases based on previous performance. For each case, residents completed criterion and disease pattern checklists and provided final diagnoses. For cases in which the resident made a diagnostic interpretation error, we evaluated the accuracy of checklist components, bias type $(\mathrm{n}=35)$, and level of training. We categorized specific case types (i.e., rare or common) and training level with specific biases and knowledge gaps and designed a forecasting checklist to assist residents and pathologists in practice. Results: Regardless of experience level, residents learned quickly to complete criterion and pattern checklists accurately but were challenged in linking histologic patterns to specific disease types ( $75 \%$ of failures). For inexperienced residents, $60 \%$ of diagnostic errors were associated with knowledge gaps and anchoring, recency, or attention biases. In more experienced residents $50 \%$ of errors were associated with expectation, over confidence, or clustering illusion bias. Errors involving special stains were associated with confirmation or gaze bias. For straightforward cases, $55 \%$ of errors were associated with anchoring, observer-expectation, or recency bias. For complex cases, $45 \%$ of errors were associated with do no harm, confirmation, or framing bias. Using forecasting, the residents were competent at recognizing knowledge gaps and difficult case biases. Residents often did not use bias-checklists in easier cases.

Conclusions: We hypothesize that a considerable proportion of diagnostic errors are linked to bias and that individual characteristics (e.g., experience) and specific scenarios are associated with specific bias types. We further hypothesize that SBME and the use of forecasting with bias-checklists has the potential to decrease diagnostic error through altering traditional cognitive patterns.

\section{Impact of Integrated Speech Recognition and Digital Dictation} Software on Turnaround Times for Surgical Pathology Specimens

VS Snyder, CL Kinonen, CEJ Johnson, BC Gleason, AB Thomas, TL Cibull. NorthShore University HealthSystem, Evanston, IL; Diagnostic Pathology Medical Group, Sacramento, CA.

Background: Integrated speech recognition and digital dictation software (ISRDDS) has been used for medical transcription in various medical fields for many years. Recently, pathology and laboratory medicine professionals have implemented this technology, but there is little literature on how it impacts pathology practices. The goal of this study is to evaluate how ISRDDS has affected turnaround times (TATs) for surgical biopsy specimens.

Design: We evaluated TATs (number of hours from accession to sign out) pre-ISRDDS (6/1/2011-8/31/2011) and post-ISRDDS (6/1/2012-8/31/2012) implementation. Specimens included in the study were all biopsies: skin, prostate cores, endometrium $(\mathrm{L}=$ Lab services, $\mathrm{H}=$ Hospital $)$, breast cores, and GI biopsies .

Results: The percentage of cases signed out at a given number of hours, pre- and postISRDDS implementation is summarized in Tables 1 and 2 , and illustrated graphically in Figure 1. For each specimen type, TATs were faster post-ISRDDS implementation. Table 1: Pre-ISRDDS. \% cases signed out at given number of hours after accessioning.

\begin{tabular}{|c|c|c|c|c|c|c|c|}
\hline \multirow[b]{2}{*}{ Specimen type } & \multicolumn{7}{|c|}{ Hours post accessioning } \\
\hline & 12 & 16 & 18 & 20 & 22 & 26 & 28 \\
\hline Skin & 0.5 & 25 & 50 & 79 & 94 & 100 & 100 \\
\hline Prostate & 0 & 18 & 43 & 63 & 74 & 100 & 100 \\
\hline Endometrium (L) & 0 & 8.5 & 27 & 51 & 72 & 100 & 100 \\
\hline Endometrium (H) & 0 & 0 & 0 & 0 & 7 & 68 & 88 \\
\hline Breast & 0 & 0 & 0 & 6 & 15 & 58 & 82 \\
\hline GI & 0 & 0 & 1 & 9 & 24 & 68 & 91 \\
\hline
\end{tabular}

$\mathrm{L}=\mathrm{Lab}$ services; $\mathrm{H}=$ Hospital

\begin{tabular}{|c|c|c|c|c|c|c|c|}
\hline \multirow[b]{2}{*}{ Specimen type } & \multicolumn{7}{|c|}{ Hours post accessioning } \\
\hline & 12 & 16 & 18 & 20 & 22 & 26 & 28 \\
\hline Skin & 5.9 & 41 & 66 & 87 & 97 & 100 & 100 \\
\hline Prostate & 5.4 & 34 & 60 & 73 & 83 & 100 & 100 \\
\hline Endometrium (L) & 0 & 40 & 65 & 83 & 94 & 100 & 100 \\
\hline Endometrium $(\mathrm{H})$ & 0 & 0 & 3.2 & 16 & 35 & 86 & 94 \\
\hline Breast & 0 & 0 & 5 & 14 & 34 & 81 & 94 \\
\hline GI & 0 & 0 & 2 & 10 & 29 & 80 & 92 \\
\hline
\end{tabular}

$\mathrm{L}=$ Lab services; $\mathrm{H}=$ Hospital 

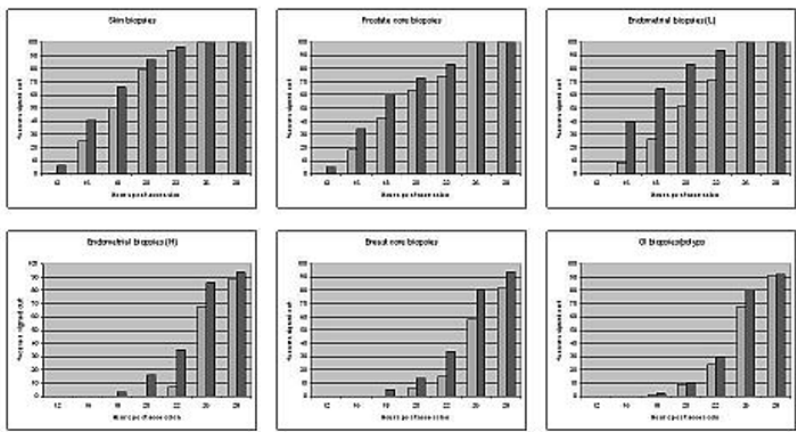

Figure $1 . \%$ of cases signed out at number of hours post accessioning
P POSt-ISRDDS

Conclusions: TATs improved after implementation of ISRDDS, most notably among breast and endometrial biopsies. The decrease in TAT was less dramatic for skin and prostate biopsies, in which TAT was already excellent, with $>80 \%$ of cases signed out in 22 hours pre-ISRDDS. However, the introduction of ISRDDS improved TATs for skin and prostate biopsies such that post ISRDDS $>50 \%$ of cases were signed out by 18 hours, which impacts patient care by allowing clinicians to provide their patients results the day after the biopsy was performed.

\section{Improving Quality and Completeness of Cancer Pathology Reporting: Results of a Large Scale 8 Year Canadian Project}

J Srigley, D Divaris, T McGowan, J Brierley, M Yurcan, J Irish, R McLeod, J Ross, $R$ Rossi, T Yardley, R Schill, M King, J Hart, D Wilson-Li, C Sawka. Cancer Care Ontario, Toronto, ON, Canada; McMaster University, Hamilton, ON, Canada; Grand River Hospital, Kitchener, ON, Canada; Credit Valley Hospital, Mississauga, ON, Canada; University Health Network, Toronto, ON, Canada; University of Toronto, Toronto, ON, Canada; Mt. Sinai Hospital, Toronto, ON, Canada; University Health Network \& Mt Sinai Hospital, Toronto, ON, Canada.

Background: Standardizing electronic pathology reporting in discrete synoptic format across a large population (13.5 million) involving over 60,000 new cancer cases per year, 116 acute care hospitals and over 400 pathologists is no small feat. This 8 year initiative improved the quality of cancer pathology reports by standardizing the content to align with the College of American Pathologists (CAP) cancer protocols and electronic checklists (eCCs). A change management strategy enabled the uptake of standardized electronic synoptic pathology reporting.

Design: The mandate to standardize electronic pathology reporting was delivered through a central agency to all 52 primary reporting facilities (a total of 116 acute care facilities). The adoption of eTools in all participating hospital laboratory information systems (LIS) provided pathologists with preformatted drop down lists based on the CAP eCCs. Phase 1 implemented 5 high volume checklists (breast, CRC, lung, prostate and endometrium). Phase 2 expanded the mandate to 63 checklists using the eCC standard. Change management strategies targeted practice change enablers (clinical leadership, KTE, audits) and system change enablers (funding, standards adoption, LIS vendor engagement, process improvement).

Results: In 2004, 95\% of acute care hospitals submitted pathology reports in nonstandardized narrative or synoptic-like formats, with completeness rates audited at $68 \%$ on the CAP Cancer Protocol standard (breast, prostate, lung, CRC). As of March $2012,97 \%$ of hospitals use discrete data field electronic pathology reporting. Over $90 \%$ of cancer resections for the mandated 63 eCCs are submitted monthly in synoptic format with a completeness rate of $>90 \%$. The data forms the basis for several clinicalpathology performance metrics.

Conclusions: The success of implementing electronic synoptic pathology reporting across a large population is attributed to the key enablers for change, especially clinical engagement and regular auditing applied at participating sites and facilitated through a central agency focusing on quality improvement.

\section{Quality Improvement in Pathology through Real-Time Labeling} R Stapp, M Haghighi, R Brown, M Czechowski, B Mahar, R Zarbo, M Tuthill. Henry Ford Hospital, Detroit, MI.

Background: We implemented a 'real-time labeling' process in the anatomic pathology department. 'Real-time labeling' is a process enabled through our laboratory information system (LIS) which allows case-by-case labeling of anatomic pathology cassettes and slides with reductions in patient specimen misidentification rates and improved efficacy in daily workflow. This process is the extension of several other cycles of improvement, encompassing five years of effort.

Design: Prior to this process, tissue cassettes were printed with case identification details using cassette printers, which required re-entering demographic and case data into dedicated software. After processing, slides were manually labeled and stained. Paper labels were batch printed and affixed onto slides. This manual process was replaced by a "bar code specified surgical pathology" workflow that automated labeling of slides and cassettes, replacing paper labels with stain resistant labels. We have continually evaluated this process by applying the plan, do, check, act (PDCA) methodology. Recently, we replaced linear bar codes with 2D bar codes as part of an upgrade of our LIS, which also included replacement of the old labeling system with 'real-time labeling'. The new functionality allowed seamless slide label printing in real-time as each histology block was scanned before sectioning. $2 \mathrm{D}$ barcode scanners were installed at individual workstations within grossing rooms, pathologists' offices, and histology lab. Results: Previously, the process of batch printing labels increased the risk of patient misidentification. While this was addressed with prior PDCA cycles, it was prone to failure in label production from cassettes and slide bar code read errors. Since, bar codes were unreliable, their use in managing cases decreased. With 'real-time labeling' the batch printing process been eliminated and misidentification rates have been further reduced from $0.62 \%$ to $0.02 \%$. Histology efficacy has increased as cassette reading defects have been eliminated, that required manual entry of case numbers. Since implementation, we have seen no internal patient identification issues due to labeling. Finally, the linear label barcode defects have been entirely eliminated by using $2 \mathrm{D}$ barcodes on slide labels. This has decreased read errors from $20 \%$ of slides on a daily basis to $0 \%$.

Conclusions: Through the use of multiple PDCA cycles we were able to improve processes throughout laboratory with the implementation of 'real-time labeling'. The changes have led to reductions in patient misidentification, increased efficiency, and improved turn-around time.

2023 The Diagnostic Black Box: Successful Integration and Performance Validation of Pathologists Using New Lung Non-Small Cell Carcinoma Classification Recommendations

ME Stevenson, P Murugan, LA Hassell. University of Oklahoma Health Sciences Center, Oklahoma City, OK.

Background: The proposed IASL/ATS/ERSIM classification of lung adenocarcinoma presents a paradigm shift for diagnostic pathologists. We sought to validate our ability to apply the new classification recommendations in reporting of non-small cell lung cancer (NSCLC) cases.

Design: A test based on the new NSCLC classification recommendations was administered to 14 pathology faculty members and senior residents or fellows prior to and after major educational interventions, which included circulation of articles, electronic presentations, and live presentations by a prominent pulmonary pathologist. Surgical and cytologic (including cell block material) reports of lung malignancies for representative periods prior and subsequent to the educational interventions were reviewed for compliance with the new recommendations. Cases were scored on a three point scale with 1 indicating incorrect terminology and/or highly inappropriate stain utilization; 2 indicating correct diagnostic terminology with suboptimal stain utilization; and 3 indicating appropriate diagnosis and stain utilization. Error type was also evaluated.

Results: The initial testing average correct score was $62 \%$, increasing to $88 \%$ following the educational interventions ( $42 \%$ improvement). Of the 54 reports evaluated preintervention 15 were scored $3 / 3$ (28\% of total reports), 31 were scored $2 / 3(57 \%)$, and 7 were scored $1 / 3(13 \%)$. Incorrect use of stains was noted in 23 of 54 cases $(43 \%)$, incorrect terminology in 15 of 54 cases $(28 \%)$, and molecular testing errors in 4 out of 54 cases (7\%). Of 34 cases in the post-intervention group, 27 were scored $3 / 3$ ( $79 \%$ of cases), 6 were scored $2 / 3(18 \%)$, and 1 was scored $1 / 3$ (3\%). Incorrect use of stains was the only error identified ( 7 out of 34 cases, representing $21 \%$ of total reports). Conclusions: The study results demonstrate marked improvement in participants' understanding and application of the new NSCLC classification recommendations sufficient to validate our use of the system in routine practice. The results also affirm the value of intensive education for validation of new classification or diagnostic algorithms.

2024 Center Duplication of Molecular Testing at a Tertiary Care Oncology MW Taggart, W-C Foo, SC Abraham. MD Anderson Cancer Center, Houston, TX. Background: Molecular testing of solid tumors is rapidly expanding due to the increased use of predictive and prognostic markers in personalized cancer therapy. In gastrointestinal (GI) carcinomas, mutation in either $K R A S$ or $B R A F$ genes predicts lack of response to cetuximab-based chemotherapy in second-line treatment of metastatic disease. Microsatellite instability (MSI) analysis by PCR and immunohistochemistry (IHC) can identify patients with Lynch syndrome and (arguably) identify tumors with lack of response to 5-FU but improved cancer-related survival. The widespread use of these markers in GI oncology can lead to unnecessary duplication of molecular testing when patients are referred to tertiary care centers.

Design: We reviewed all molecular tests requested by GI oncologists or oncologic surgeons for clinical/non-investigational purposes on 100 consecutive patients with colorectal or small bowel carcinomas at our cancer center during 2012. Available scanned copies of original pathology reports, outside laboratory tests, and outside and inside clinical notes were carefully reviewed to determine whether the requested tests were previously performed. In instances where testing was mentioned in the outside clinic notes but results were not documented, phone calls were placed to determine if the testing actually took place.

Results: 217 molecular tests were requested in these 100 patients, including 49 for $K R A S, 36$ for $B R A F, 64$ for MSI analysis by PCR, 65 for MSI analysis by IHC (MLH1, MSH2, MSH6 and PMS2) and 3 for $M L H 1$ promotor methylation to help distinguish sporadic MSI-high carcinoma with MLH1 protein loss from Lynch syndrome-associated cancer. Unnecessary molecular testing was requested in 7 (14\%) KRAS, 5 (14\%) BRAF, $3(5 \%)$ PCR for MSI, 9 (14\%) IHC for MSI, and 1 (33\%) MLHI promotor methylation. Among this group of unnecessary requests, testing was variably: 1) already performed at an outside CLIA-certified laboratory on the same or equivalent specimen $(\mathrm{n}=12), 2)$ scientifically invalid (e.g., request for $B R A F$ analysis in a specimen known to be $K R A S$ mutated) $(\mathrm{n}=4)$, or 3 ) requested twice for in-house testing (e.g., separate requests at different times by an oncologist or his/her physician's assistant) $(n=9)$. 
Conclusions: Given the time-consuming nature and high cost of molecular testing, duplication or unnecessary testing in $12 \%$ of GI carcinomas can significantly drive up the costs of specialized medical care. These results support the role of pathologists in screening molecular testing requests, the incorporation of outside laboratory results into pathology reports, and the push for widespread use of electronic medical records.

\section{Validation of Remote Robotic Microscopy Using the iScan} Coreo Au Platform

MJ Thrall, H Takei, AL Rivera, SZ Powell. Methodist Hospital, Houston, TX

Background: Remote robotic microscopy (RRM) allows a remote pathologist to manipulate and view a slide via the internet. This technology is useful for intraoperative assessments (IA) when whole slide imaging (WSI) would be too time-consuming. Touch/ squash preparations are especially difficult for WSI because of the widely dispersed and 3-dimensional diagnostic material.

Design: Our pathology department receives occasional requests for neuropathology IA from a branch hospital with no neuropathologist. We validated the iScan Coreo $\mathrm{Au}$ "Live Mode" RRM for touch/squash preparations to address this problem. 100 cases were selected, including 75 consecutive neuropathology cases with IA and 25 consecutive general surgical pathology cases with touch preparation IA. Three neuropathologists and one general surgical pathologist participated; each viewed 25 cases. Half of the cases were viewed on glass slides by conventional microscopy and half by RRM, followed by a delay of at least 3 weeks, after which the cases were viewed by the other modality. Diagnoses and times spent viewing were recorded. Intraobserver variation was analyzed. Results: Of the 100 cases, 78 showed perfect intraobserver agreement and 10 more showed minor discrepancies (less specific diagnoses). There were 12 cases with significant discrepancies (listed below)

Intraobserver Discrepancies

\begin{tabular}{|l||l||l|}
\hline Glass Slide & Remote Robotic Microscopy & $\begin{array}{l}\text { Final Permanent Section } \\
\text { Diagnosis }\end{array}$ \\
\hline Consistent with meningioma & Scant interpretable material - defer & Atypical meningioma \\
\hline Glial neoplasm & Mildly hypercellular glial tissue & Glioblastoma \\
\hline Gliotic brain & Possible infarct & Consistent with infarct \\
\hline High grade astrocytoma & Ependymoma & Ependymoma \\
\hline High grade astrocytoma & Non-diagnostic - defer & Glioblastoma \\
\hline High grade glioma & Schwannoma & Large B-cell lymphoma \\
\hline Infiltrating glioma & Mildly hypercellular glial tissue & Glioblastoma \\
\hline Low grade glioma & Hemorrhagic lesion & Pleomorphic \\
xanthoastrocytoma
\end{tabular}

The average time to reach a diagnosis by RRM was 128 seconds versus 44 seconds for glass slides. Pathologists found RRM to be frustrating. It was difficult to decide what part of the slide to look at on high power using the RRM thumbnail images.

Conclusions: Overall, RRM performs well for touch/squash preps. RRM is cumbersome relative to glass slides, and is more challenging for the pathologist resulting in less decisive interpretations, but is still acceptable for remote IA.

2026 Validation of Two Whole Slide Imaging Scanners Based on the Draft Guidelines of the College of American Pathologists

MJ Thrall. Methodist Hospital, Houston, TX.

Background: Whole slide imaging (WSI) converts glass slides into digital images that can be viewed remotely. We have four branch hospitals dependent on a centra academic hospital for slide production and expert consultation. We intend to use WSI to avoid glass slide courier delays. We validated using the draft guidelines released by the College of American Pathologists in 2011.

Design: 100 consecutive cases were selected from the following categories that mimic the intended use: consults, frozen sections, malignancies, and special or immunohistochemical stains. Key slides were selected for each case. The slides were scanned at 20x magnification using standard focus and no Z-axis on 2 Ventana iScan Coreo Au scanners (the same 100 cases scanned twice). Pathologists viewed half of the cases as glass slides first and half as WSI first, then switched to the other modality after a delay of at least 3 weeks. Brief history was provided. Diagnoses were documented and intraobserver agreement compiled after the completion of all slide and WSI review. Results: 23 pathologists participated; most viewed 9 cases. Intraobserver agreement was seen in 165 cases $(82.5 \%)$, with disagreement in 35 cases $(17.5 \%)$. Our most striking finding was that 10 cases $(5 \%)$ showed disagreement between WSI and glass slides because of focal findings not seen in the WSI modality.

Focal Findings Not Seen by Whole Slide Imaging

\begin{tabular}{|c|c|c|c|}
\hline $\begin{array}{l}\text { Specimen } \\
\text { Source }\end{array}$ & Glass Slide Diagnosis & WSI Diagnosis & $\begin{array}{l}\text { WSI Scanner With } \\
\text { Unseen Finding }\end{array}$ \\
\hline Bone & Osteomyelitis & Osteoarthritis & Scanner \#1 \\
\hline Breast & Atypical lobular hyperplasia & Negative & Both scanners \\
\hline Colon & Focal active colitis & Negative & Both scanners \\
\hline Esophagus & Candida seen & No Candida seen & Both scanners \\
\hline Liver & Iron 1-2+ & No increase in iron & Scanner \#2 \\
\hline Stomach & Suspicious for adenocarcinoma & Negative & Scanner \#1 \\
\hline Stomach & Chronic active gastritis & Chronic inactive gastritis & Scanner \#1 \\
\hline
\end{tabular}

\section{WSI $=$ Whole Slide Imaging}

Conclusions: Overall, WSI performs well when compared to glass slides in our validation. The most concerning cause of intraobserver variability was diagnostically important small foci seen on glass slides but not WSI. Relative lack of familiarity with WSI, the slowness of WSI which discourages thorough scanning, and the "low stakes" setting of validation may all have contributed to this problem. However, the lack of good definition of the images at high power must be considered as a factor. Images produced with the fastest scan times (20x magnification and automatic focus) may not be sufficient for a confident negative diagnosis, especially in cases with subtle findings.
2027 Proficiency Testing for Mismatch Repair Immunohistochemistry in Canada

E Torlakovic, B Clarke, CJ Streutker, S Hafezi-Bakhtiari, SE Kalloger, M Anna Marie, $R$ Wolber, B Gilks, A Pollett. University Health Network, University of Toronto, Toronto, ON, Canada; St. Michael Hospital/University of Toronto, Toronto, ON, Canada Vancouver Costal Health, Vancouver, BC, Canada; University of British Columbia, Vancouver, BC, Canada; Mt. Sinai Hospital/University of Toronto, Toronto, ON, Canada Background: Current methods (clinical history and tumor morphology) to predict MMR deficiency (dMMR) are suboptimal. In view of recommendations of reflex MMR-IHC screening policies and in recognition of the growing predictive role of dMMR we undertook to determine the current quality indicators of dMMR testing in Canadian laboratories. This is part of an endeavor by the Canadian Association of Pathologists/ Association Canadienne des pathologistes (CAP-ACP) National Standards Committee for Complex Laboratory Testing to introduce a Canadian consensus statement and national guideline on dMMR testing. The EQA run was conducted by the Canadian Immunohistochemistry Quality Control, which is partly supported by Canadian Partnership Against Cancer (CPAC).

Design: TMA was constructed from the 39 tumor samples with known germline status. Histologically normal tissues were included as additional tissue cores from each sample. Unstained slides were sent to 14 laboratories that were identified as performers of the IHC testing for MLH1, MSH2, MSH6, and PMS2. Expert assessment was conducted by five experienced pathologists. Percent correct results, false-positive and false-negative rate, specificity and sensitivity were calculated.

Results: Success with histologically normal tissues had linear-by-linear association with specificity with tumor samples $(\mathrm{p}=0.002)$. False absence of nuclear staining (FANS) in the tumor strongly correlated with the absence of staining in the normal tissue ( $\mathrm{r}=0.959, \mathrm{p}<0.0001$, Pearson's $\mathrm{R}$ for all four tests). Sensitivity and specificity of each test are shown in Table 1.

\begin{tabular}{|c|c|c|c|c|}
\hline & MLH1 & MSH2 & MSH6 & PMS2 \\
\hline Sensitivity & $88-100(96)$ & $100(100)$ & $93-100(99)$ & 90-100(99) \\
\hline Specificity & 64-100(96) & $75-100(93)$ & $38-98(73)$ & $19-100(86)$ \\
\hline
\end{tabular}

False-negative (left) and false-positive (right) rates are illustrated in Fig.1.

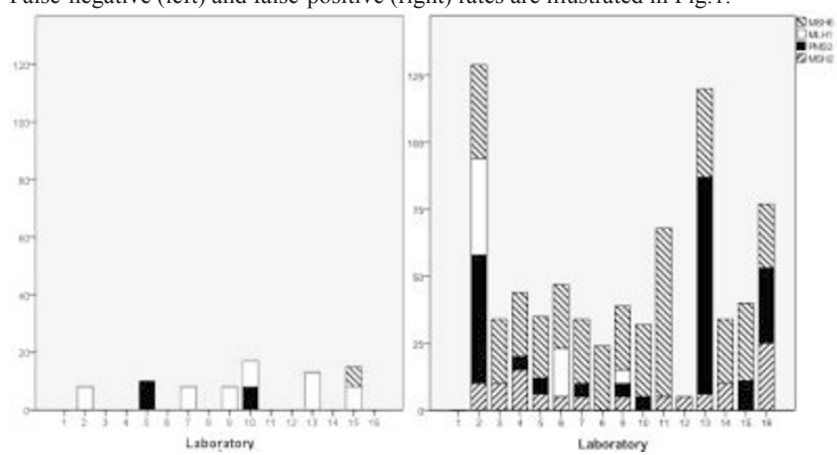

Conclusions: MMR-IHC testing in Canada is characterized by high sensitivity. The most challenging test was PMS2 with poor specificity. Normal tissues should always be evaluated as internal controls as they highly correlated with test specificity.

2028 Rapid On-Site Evaluation (ROSE) for Endobronchial UltrasoundGuided Fine Needle Aspiration (EBUS-FNA): An Inter-Institutional Comparative Study

PA VanderLaan, KJ Brister, KP Rao, JD Marotti, HH Wang. Beth Israel Deaconess Medical Center \& Harvard Medical School, Boston, MA; Dartmouth-Hitchcock Medical Center, Lebanon, NH.

Background: ROSE is generally considered a beneficial practice to improve diagnostic yield of EBUS-FNAs; however, complicating factors are often not considered. To address this issue, a study of two academic medical centers with different ROSE practice patterns was performed to probe the impact of ROSE on EBUS-FNA procedures and subsequent outcomes.

Design: Following IRB approval, retrospective data were collected from all EBUSFNAs performed over a one-year time period (8/2011-8/2012) at two tertiary-care Academic Medical Centers: AMC-A where ROSE is only occasionally utilized for EBUS-FNA procedures, and AMC-B where ROSE is routinely used for EBUS-FNA procedures. Variables investigated included $\%$ of repeat procedures and ROSE, site and number of stations sampled, procedure time, and distribution of final diagnoses. Statistical significance was determined by t-test for continuous variables and by chisquared test for categorical variables. 
Results: Study results are presented in the table below.

\begin{tabular}{|c|c|c|c|}
\hline Variable & Institution & & p value \\
\hline & AMC-A & AMC-B & \\
\hline Procedure \# & 304 & 187 & \\
\hline Repeat procedures & $10(3.3 \%)$ & $5(2.7 \%)$ & 0.7 \\
\hline ROSE done & $28(9.2 \%)$ & $183(98 \%)$ & $<0.001$ \\
\hline Specimen type & & & $<0.001$ \\
\hline Mass only & $32(11 \%)$ & $31(17 \%)$ & \\
\hline Lymph nodes only & $212(70 \%)$ & $146(78 \%)$ & \\
\hline Both & $60(20 \%)$ & $10(5.4 \%)$ & \\
\hline Number of sampled nodal stations & & & $<0.001$ \\
\hline None & $32(11 \%)$ & $31(17 \%)$ & \\
\hline 1 & $77(25 \%)$ & $137(73 \%)$ & \\
\hline 2 & $85(28 \%)$ & $18(9.6 \%)$ & \\
\hline 3 & $73(24 \%)$ & $1(0.5 \%)$ & \\
\hline 4 or more & $37(12 \%)$ & $0(0 \%)$ & \\
\hline Mean time per procedure (min) & $63(15-162)$ & $63(14-142)$ & 0.88 \\
\hline Mean time: 1 site sampled & $56(15-127)$ & $58(14-142)$ & 0.52 \\
\hline Mean time: 2 sites sampled & $58(25-130)$ & $90(42-142)$ & $<0.0001$ \\
\hline Mean time: $3+$ sites sampled & $72(18-162)$ & $105(87-117)$ & 0.03 \\
\hline Final Diagnosis & & & 0.57 \\
\hline Non-diagnostic & $22(7.2 \%)$ & $8(4.3 \%)$ & \\
\hline Negative & $111(37 \%)$ & $66(35 \%)$ & \\
\hline Atypical & $22(7.2 \%)$ & $11(5.9 \%)$ & \\
\hline Suspicious & $6(2.0 \%)$ & $3(1.6 \%)$ & \\
\hline Positive & $143(47 \%)$ & $99(53 \%)$ & \\
\hline
\end{tabular}

Conclusions: The routine use of ROSE for EBUS FNA procedures tends to lead to slightly lower non-diagnostic rates; however, ROSE significantly prolongs the procedure time relative to the increasing number of sites sampled. Without ROSE, a more complete mediastinal staging (i.e. more lymph node stations) can be performed without significantly increasing the procedure time. From a cytopathology management perspective, routine use of ROSE can represent a significant personnel cost. With an increasingly likely bundled payment environment for health care reimbursement, further study is needed to determine the optimal utilization of ROSE to maximize both the quality and efficiency of patient care.

\section{Turnaround Time for Large or Complex Specimens in Surgical Pathology: A Q-Probes Study of $\mathbf{5 6}$ Institutions}

KE Volmar, MO Idowu, DS Karcher, RE Nakhleh. Rex Pathology Associates, Raleigh, $\mathrm{NC}$; Virginia Commonwealth University, Richmond, VA; George Washington University Medical Center, Washington, DC; Mayo Clinic, Jacksonville, FL.

Background: Turnaround time (TAT) for large or complex surgical pathology specimens is an indicator of efficiency in anatomic pathology and may affect coordination of patient care. This study aimed to develop benchmarks for TAT of these cases and to identify practice characteristics that may influence TAT.

Design: Participants in a 2012 Q-Probes quality improvement program of the College of American Pathologists were asked to retrospectively review all surgical pathology cases over the prior six months to identify up to 50 cases coded as CPT code 88307 (excluding biopsies) and/or 88309. Participants reported the times and dates of accessioning and final report sign out.

Results: 56 institutions reported on 2,763 large or complex cases. The case mix was $70 \%$ CPT code 88307 and $30 \%$ CPT code 88309 . Cases requiring special handling comprised $51.5 \%$, while $48.5 \%$ were characterized as routine. Actual TAT (not working days) was calculated from reported dates and times of accessioning and final sign out. For all cases the median turnaround time was 3.02 days. TATs by institutions and by case type are included in the table below.

Turnaround Time for Large or Complex Surgical Pathology Cases (in actual days)

\begin{tabular}{|l|l||l|l||}
\hline \hline & 10 th percentile & 50 th percentile & 90 th percentile \\
\hline Institutional TAT & 6.23 & 2.72 & 1.22 \\
\hline Routine handling cases & 5.99 & 1.94 & 0.98 \\
\hline Special handling cases & 9.11 & 4.13 & 1.33 \\
\hline
\end{tabular}

Significantly longer TAT occurred in governmental institutions (median 6.06 vs 2.13, $P<.001)$ and in institutions that mandate overnight fixation for some specimen types (median 3.83 vs $2.07, P=.03$ ). Case characteristics associated with significantly longer TAT were as follows: CPT code 88309 vs 88307 (median 3.99 vs $2.82, P<.001$ ), special handling (median 4.13 vs $1.94, P<.001$ ), cases with frozen section (median 3.38 vs $2.92, P<.001)$, radical cancer resection vs other resection types $(P<.001)$, and malignant vs non-malignant cases $(P<.001)$. The most common forms of special handling were extended fixation (27.7\%) and use of immunohistochemistry (27.6\%). TAT was not significantly affected by either training programs or routine weekend sign out. Conclusions: This study provides benchmark data for TAT in large or complex surgical pathology specimens. Turnaround time was good overall but the range among participating institutions was wide.

\section{Surgical Pathology Report Defects: A Q-Probes Study of 73} Institutions

KE Volmar, MO Idowu, JL Hunt, FA Meier, RE Nakhleh. Rex Pathology Associates, Raleigh, NC; Virginia Commonwealth University, Richmond, VA; University of Arkansas for Medical Sciences, Little Rock, AR; Henry Ford Hospital, Detroit, MI; Mayo Clinic, Jacksonville, FL.

Background: The rate of surgical pathology report defects is an indicator of quality and it affects clinician satisfaction. This study aimed to establish benchmarks for defect rates and defect fractions and to identify practice characteristics that may influence those rates. Design: Participants in a 2011 Q-Probes quality improvement program of the College of American Pathologists were asked to prospectively review all surgical pathology reports that underwent changes to correct various defects. Cases were collected for three months or until 50 reports were identified. Details regarding the defects were reported.
Results: 73 institutions reported on 1,688 report defects discovered in 360,218 accessioned cases, for an overall defect rate of 4.7 per 1,000 cases. Institutional defect rates are provided in the table below.

\begin{tabular}{|c|c|c|c|c|c|}
\hline Percentile & 10 th & 25 th & 50 th & 75 th & 90th \\
\hline $\begin{array}{l}\text { Defects per } \\
1.000 \text { cases }\end{array}$ & 13.5 & 8.9 & $\mid 5.7$ & 2.5 & 0.9 \\
\hline
\end{tabular}

Of the reports with defects, the distribution of defect type was as follows: $14.6 \%$ misinterpretations, $13.3 \%$ misidentifications, $13.7 \%$ specimen defects, $58.4 \%$ other defects. Overall, defects were most often detected by pathologists $(47.4 \%)$, followed by clinicians $(22.0 \%)$, but there were differences in who discovered various defect types. Specifically, compared to other personnel, pathologists more often detected misinterpretations $(73.5 \%, P<.001)$ and specimen defects $(82.7 \%, P<.001)$, while misidentifications were most often discovered by clinicians $(44.6 \%, P<.001)$. Significantly higher defect rates were seen in institutions with a pathology training program ( 8.5 vs 5.0 per $1,000, P=.01)$. A lower rate of misidentification was seen when all malignancies were reviewed by a second pathologist before sign out ( $0.0 \mathrm{vs}$ 0.6 per $1,000, P<.001)$, and a lower rate of defects from specimen issues was seen with intradepartmental review of difficult cases after sign out ( 0.0 vs 0.4 per $1,000, P=.02$ ). Institutions that review a set percentage of cases after sign out tended to have higher defect rates (median 6.7 vs 3.8 per $1,000, P=.10$ ).

Conclusions: This study provides benchmarking data on report defects and defect fractions. The study reveals a higher rate of report defects and a higher fraction of misinterpretations than prior studies.

\section{Institution of $\mathbf{2 4}$ Hour Histology Processing and the Impact on} Turn-around-Time

LT Wiehagen, RD Wall, SM Kelly, SA Yousem, AL Piccoli, AA Green, AV Parwani, L Pantanowitz, S Navina. UPMC Presbyterian Shadyside, Pittsburgh, PA.

Background: Expeditious processing of pathologic specimens, to make slides available in a timely manner for pathologist review, enables the pathologist to render diagnosis in a timely manner for patient care. This dramatically decreases the time it takes for clinicians to begin treatment of patients. This project describes the effects of moving to a 24 hour work cycle in our institute's Histology Laboratory. It compares similar time periods pre and post implementation to gauge effectiveness.

Design: Prior to implementation, the Histology Laboratory operated on a daylight shift and serviced two hospital campuses. Specimens were processed on two runs set up during the day and ran automatically during the night when no staff was on site. During the day, histology staff embedded blocks, made slides and performed stains on the specimens processed the night before. After months of planning, a 24 hour work cycle was implemented and a new workflow was designed. This included two additional work shifts - an evening and night shift. This change allowed continuous specimen processing, embedding, cutting and staining.

Results: The project measured the turnaround time by each bench from accession to sign-out prior to implementation and post implementation as illustrated in Table 1. TAT was significantly reduced in most benches.

\begin{tabular}{|c|c|c|c|c|}
\hline $\begin{array}{l}\text { Center of } \\
\text { Excellence (COE) }\end{array}$ & Pre 24Hr (days) & $\begin{array}{l}24 \mathrm{Hr} \text { Process } \\
\text { (days) }\end{array}$ & $\%$ Change & $\begin{array}{l}\text { Avg. Minutes } \\
\text { Saved Per Case }\end{array}$ \\
\hline ENT & 2.91 & 2.37 & $-18.52 \%$ & 266.67 \\
\hline GU & 2.37 & 2.75 & $15.76 \%$ & -226.92 \\
\hline GI - BIGS & 2.77 & 2.64 & $-4.95 \%$ & 71.31 \\
\hline GI - Quicks & 1.40 & 1.38 & $-1.42 \%$ & 20.38 \\
\hline Neuropath & 2.53 & 2.47 & $-2.34 \%$ & 33.63 \\
\hline PUH Cyto & 1.78 & 1.40 & $-21.18 \%$ & 304.99 \\
\hline SHY Cyto & 1.63 & 1.57 & $-3.39 \%$ & 48.82 \\
\hline BST & 2.24 & 2.14 & $-4.59 \%$ & 66.16 \\
\hline Thoracic & 1.67 & 1.46 & $-12.98 \%$ & 186.89 \\
\hline Transplant & 1.48 & 1.35 & $-9.13 \%$ & 131.47 \\
\hline Overall & 2.03 & 1.87 & $-7.93 \%$ & 114.25 \\
\hline
\end{tabular}

Conclusions: There are a litany of variables that can affect TAT within a pathology department that include both professional and technical components. Acknowledging other variables, a 24 hour processing can significantly decrease the TAT of Histology processing, affecting total specimen sign-out TAT Moving to the new processing schedule also allowed for additional capacity within the lab. Since the lab services two campuses, the greater impact was seen on the specimens that remained on the campus which houses the Histology lab.

\section{$2032 \quad$ Impact of Streamlined Adverse Event Entry}

LT Wiehagen, SM Kelly, GH Murdoch, AL Piccoli, SA Yousem, L Pantanowitz, AV Parwani, RR Seethala. UPMC Presbyterian Shadyside, Pittsburgh, PA.

Background: Entry of Adverse Events (AE) is the primary mechanism for the recording of in process "defects" in Anatomic Pathology. Ideally, this should be a rapid, easy process in order to maximize participation and thus provide a more accurate assessment of the prevalence of process defects. Through software enhancements and structured revisions to the lengthy and tedious $\mathrm{AE}$ and resolution dictionaries in the laboratory information system (LIS), our aim was to increase the participation of pathologists and technical staff to assist in quality improvement and patient safety.

Design: Modifications were made in our LIS (CopathPlus) to eighty one windows and tabs to enhance and streamline reporting. A new dictionary was designed which centered around workflow and phases of testing resulted in 8 main categories (assigned alphanumeric codes for quick retrieval). Branching subcategories of the most critical events in each category were created and a free text field was expanded to allow for a detailed description of the event. Each main category was painted on the top of the AE window in CoPathPlus for reference. Once a main AE category was selected a pop-up window displayed only the associated subcategories for easy selection. A comparison 
between the AE entry rates per month was performed pre and post implementation. Results: There was an overall reduction from 254 dictionary terms to 63 terms. With the branching subcategory display, the effective number of terms that the end user has to sort through decreased from 254 to between 8 and 14 options. AE entry rates were measured pre and post implementation and illustrated in Table 1. The comparison of the two time periods showed a very dramatic increase of the recording of "defects" post implementation. Overall, there was a $124.4 \%$ increase when the two time periods were compared.

\section{Table 1}

\begin{tabular}{|l||l||l|l|}
\hline Month & Usage PRE Implementation & Usage POST Implementation & Percent Change \\
\hline 1 & 188 & 425 & $126.1 \%$ \\
\hline 2 & 193 & 487 & $152.3 \%$ \\
\hline 3 & 238 & 416 & $74.8 \%$ \\
\hline 4 & 255 & 512 & $100.8 \%$ \\
\hline 5 & 257 & 436 & $69.6 \%$ \\
\hline 6 & 183 & 472 & $157.9 \%$ \\
\hline 7 & 171 & 452 & $164.3 \%$ \\
\hline 8 & 156 & 359 & $130.1 \%$ \\
\hline 9 & 96 & 367 & $282.3 \%$ \\
\hline 10 & 123 & 523 & $325.2 \%$ \\
\hline 11 & 281 & 356 & $26.7 \%$ \\
\hline
\end{tabular}

Conclusions: A structured, workflow-based dictionary resulted in a large and sustained increase in AE reporting. This overall process improvement can potentially enhance the recognition of defects that may impact patient safety and error reduction in the pathology laboratory.

2033 Influence of External Factors on Frozen Section Performance JA Wisell, SB Sams. University of Colorado School of Medicine, Aurora, CO.

Background: Pathologists spend their days making decisions, mostly about the significance of histologic features observed during the routine practice of microscopic examination. Recently some have proposed that humans may have an individual capacity for decision-making throughout the day and once exhausted, the quality of this capacity degrades. Here we investigate whether external factors, as suggested by the concept of 'decision fatigue,' may impact upon the ability of a pathologist to render the best possible diagnosis at the time of frozen section (FS).

Design: We evaluated all FS performed within a six-month period at our tertiary level hospital and cancer center. For each FS several features were recorded including the time of day, if the FS diagnosis was discrepant from the final diagnosis and if the diagnosis was deferred at the time of FS. The discrepancies and deferrals were compared to the time of day that they occurred.

Results: Discrepancies and deferrals show a non-uniform frequency throughout the day, which demonstrate a similar pattern of occurrence, showing two periods of increased frequency.

\begin{tabular}{|l||l||l||l||l||}
\hline Time & FS volume & Discrepancy (\%) & Deferral (\%) & Discrepancy or Deferral (\%) \\
\hline$<8$ & 112 & 0 & 0 & 0 \\
\hline $8-9$ & 178 & 3.6 & 0 & 3.5 \\
\hline $9-10$ & 152 & 3.9 & 2.2 & 6.1 \\
\hline $10-11$ & 131 & 7.9 & 1.3 & 9.2 \\
\hline $11-12$ & 110 & 3.1 & 4.6 & 7.6 \\
\hline $12-13$ & 92 & 2.7 & 1.0 & 3.6 \\
\hline $13-14$ & 89 & 3.3 & 1.1 & 4.3 \\
\hline $14-15$ & 78 & 4.5 & 6.2 \\
\hline $15-16$ & 43 & 7.7 & 5.1 & 12.8 \\
\hline $16-17$ & 34 & 4.7 & 4.7 & 9.3 \\
\hline $17-18$ & 16 & 8.8 & 3.9 \\
\hline
\end{tabular}

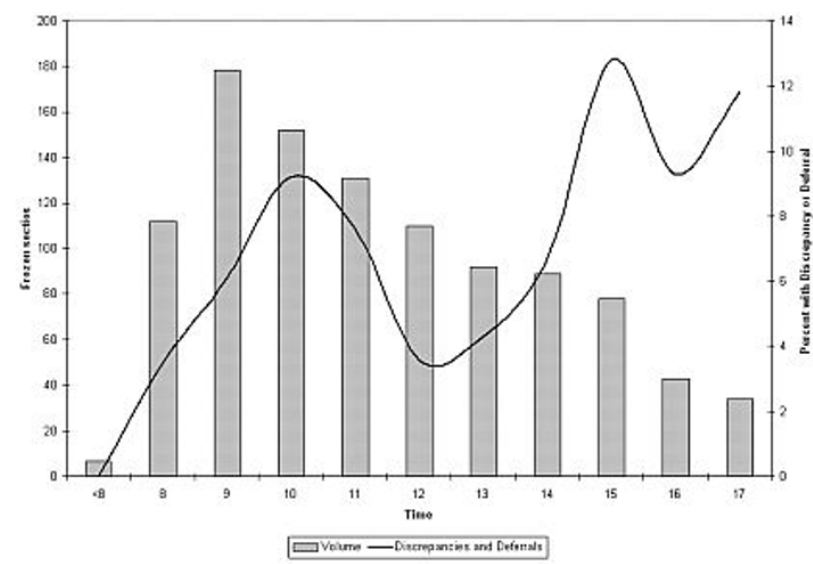

Conclusions: The first period with an increased frequency of discrepancies and deferrals occurs between 10 and $11 \mathrm{am}$, which follows the busiest period that occurs during the preceding hour. The second period of increased frequency is at the end of the day. The concept of decision fatigue could provide an explanation for this pattern, with increasing discrepancies and deferrals following the morning period of frequent decision-making and again after a full day of decisions. We acknowledge that this may not be the only explanation for our observed pattern of frozen section performance and further investigation will provide additional insight into the factors that underlie these findings. More importantly, further elucidation of influential external factors may provide opportunities for performance improvement initiatives.
2034 Is It Helpful to Further Sub-Classify Thyroid FNA Specimens Diagnosed as Atypia of Undetermined Significance/Follicular Lesion of Undetermined Significance? An Institutional Experience

LQ Wong, VA LiVolsi, ZW Baloch. Hospital of the University of Pennsylvania, Philadelphia, PA.

Background: The overall malignancy rate for the thyroid fine-needle aspiration (FNA) diagnosed as atypia of undetermined significance/follicular lesion of undetermined significance (AUS/FLUS) ranges from 5-30\%. It has been recommended to sub-classify AUS/FLUS diagnosis for better patient triage (repeat FNA vs. surgical excision). At our institution, all AUS/FLUS cases include a note regarding the cytomorphologic findings and suspicion for or against a neoplastic process. In this study, we present our experience with cases diagnosed as AUS/FLUS with sub-classifiers.

Design: A search of our laboratory information system was performed to identify all in-house thyroid FNA cases diagnosed as AUS/FLUS from 2008-2011. The data was collected and characterized by patient demographic information, cytopathology diagnosis with sub-classifiers and follow up.

Results: The case cohort included 348 cases diagnosed as AUS/FLUS in 87 males and 261 females. These were sub-classified into one of the following subcategories (SC): SC1 - favor benign, however, a follicular neoplasm (FN) could not be excluded due to increased cellularity; SC2 - specimens with focal nuclear overlapping and crowding; SC3 - scant specimens with focal nuclear overlapping and crowding; SC4 specimens with focal nuclear overlapping and crowding in a background of lymphocytic thyroiditis; SC5 - few cells with features suspicious for papillary thyroid cancer (PTC), SC6 - specimens in which a FN cannot be excluded (with miscellaneous morphologic descriptors). Of the 348 cases, 13 (4\%) were sub-classified as SC1, $127(36 \%)$ as SC2, $128(37 \%)$ as SC3, $52(15 \%)$ as SC4, $17(5 \%)$ as SC5 and $11(3 \%)$ as SC6. Histologic follow-up was available in $139(40 \%)$ cases; $45(32 \%)$ cases were found to be malignant. The malignancy rate for each subcategory was: SC1 - 0\%, SC2 - 36\%, SC3 - 33\%, SC4 - 22\%, SC5 - $7 \%$ and SC6 - $2 \%$.

Conclusions: Sub-classifying AUS/FLUS diagnosis may prove to be helpful in the management of patients with thyroid nodules.

\section{Techniques}

\section{Performance Evaluation Comparison of Three Commercially} Available PCR-Based KRAS Mutation Testing Platforms

JA Adams, KM Post, SA Bilbo, X Wang, JD Sen, AJ Cornwell, AJ Malek, L Cheng. Indiana University School of Medicine, Indianapolis, IN.

Background: The identification of KRAS mutations in patients with certain types of cancer, including colonic adenocarcinoma and non-small cell lung carcinoma has become increasingly important as these patients are contraindicated from receiving EGFR-targeted therapies. Several PCR-based tests are commercially available for KRAS mutation testing including Applied Biosystems KRAS Mutation Analysis on the ABI 3130xl, Qiagen therascreen KRAS RGQ PCR on the Rotor-Gene Q MDx and Qiagen KRAS Pyro on the PyroMark Q24, however these tests have not been compared side by side. The purpose of this study was to evaluate the performance characteristics and workflow for three PCR-based methods of detecting KRAS mutation status.

Design: We performed three of the commercially available PCR-based techniques for detecting the KRAS mutation, Applied Biosystems KRAS Mutation Analysis on the ABI 3130xl, Qiagen therascreen KRAS RGQ PCR on the Rotor-Gene Q MDx and Qiagen KRAS Pyro on the PyroMark Q24, on 188 patient samples and Acrometrix standards. The patient samples were of varying tissue types including colorectal, pancreatic, lung, omentum, and diaphragm. The results of each test for every specimen were documented and the three techniques were evaluated for sensitivity, specificity, positive predictive value, negative predictive value, accuracy, workflow, and cost.

Results: All of the 188 samples run were successful, with $29 \%$ being positive for the KRAS mutation. Of the positive tests, Applied Biosystems detected $84 \%$ of the positive cases, whereas Therascreen RGQ and Pyro detected $100 \%$ of the positive cases. In cases of discrepancy between Applied Biosystems and Therascreen RGQ, Pyro agreed with Therascreen RGQ 95\% of the time. Therascreen RGQ and Pyro, were comparable in terms of sensitivity, specificity, positive predictive value, negative predictive value, and accuracy, with all values being $100 \%$. All three techniques accurately identified the appropriate mutation in the known control specimens.

Conclusions: All three tests are relatively comparable for detecting the KRAS mutation, with Applied Biosystems having a slightly lower sensitivity, negative predictive value, and accuracy than Therascreen RGQ and Pyro.

\section{6}

Unexpected PAX8 Reactivity in Metastatic High Grade Breast Cancer

KH Adamson, KH Allison, PE Swanson, SM Dintzis, MH Rendi. University of Washington, Seattle, WA.

Background: Metastatic ovarian serous carcinoma and high grade breast carcinoma can be challenging to separate on histologic grounds and treatment options for these neoplasms can vary widely. PAX8 is a lineage-restricted transcription factor that is expressed in genitourinary, thyroid and Mullerian tract epithelial neoplasms, and PAX8 immunohistochemistry (IHC) is often used to differentiate between metastases from high grade breast carcinoma and ovarian serous carcinoma. Previous studies have shown that primary breast carcinomas do not express PAX8 regardless of histologic grade, but data regarding PAX8 expression in metastatic breast carcinoma are limited. Design: We evaluated 44 Nottingham grade 3 of 3 breast carcinomas (22 primary lesions and 22 metastatic lesions) for expression of PAX8 by IHC using a rabbit polyclonal antibody (Cell Marque; 1:50) and a polymer-based detection system. All 